\title{
Complex spaces and nonstandard schemes
}

\author{
Adel Khalfallah \\ SIEGMUND KOSAREW
}

\begin{abstract}
Nonstandard mathematics furnishes a remarkable connexion between analytic and algebraic geometry. We describe this interplay for the most basic notions like complex spaces/algebraic schemes, generic points, differential forms etc. We obtain - by this point of view - in particular new results on the prime spectrum of a Stein algebra.

2000 Mathematics Subject Classification 32C15, $14 \mathrm{~A} 15$ (primary); 32E10, 26E35, 58AQ10 (secondary)

Keywords: complex spaces, nonstandard schemes, generic points, ultraproducts of algebras, analytic Nullstellensatz, Stein algebras, internal polynomials
\end{abstract}

\section{Introduction}

The methods of nonstandard mathematics are in general ignored in analytic and algebraic geometry. Only some very specific applications of model theory are used to be known as for instance the Lefschetz principle, the theorem of Tarski-Seidenberg or some simple proofs of Hilbert's Nullstellensatz. Our Leitmotif is quite different and can be summarized by the following statements

- holomorphic functions (or convergent power series) should be (the standard part of) polynomials of infinite (or hyperfinite) degree

- complex spaces should be seen as hyperalgebraic schemes

- generic points of irreducible complex spaces/schemes should be certain nonstandard points

- describe differential forms as functions taking infinitesimal values (Leibniz' vision)

- replace always "countable" by "hyperfinite".

This program is achieved in our paper. Clearly, other more specific problems and conjectures in analytic/algebraic geometry can be reformulated by our "nonstandard" point of view, especially those which involve the word "infinity". 
We briefly describe the essential content of this paper. One of our fundamental constructions is that of a category of certain ringed spaces, called bounded schemes, which contains the category of algebraic $\mathbb{C}$ - schemes and which admits an essentially surjective functor, called the standard part functor, to the category of complex spaces. We thus generalize the usual passage from a nonstandard (bounded) real number to its standard part (a non constructive and non trivial procedure). The advantage of this new more algebraic category is that it allows us to apply many constructions of standard algebraic geometry which are not evident in the analytic context. We obtain analytic results just by taking the standard part functor. The essential surjectivity of the last one means, roughly speaking, that we replace holomorphic functions by nonstandard polynomials (also called internal polynomials) of some hyperfinite degree (which we can fix a priori). The polynomials obtained in this way are called bounded since they map bounded (nonstandard points) into themselves. Analogously to the classical algebraic case, we can extend sometimes holomorphic maps to compactifications in our nonstandard algebraic setting.

By a new version of what should be the meaning of a point in a complex space, namely a nonstandard one, we are able to prove that any prime ideal (not necessarily closed) of a Stein algebra which satisfies a Nullstellensatz, involving nonstandard points, is the zero set of some (non unique) nonstandard point. Especially, we can describe geometrically all maximal ideals of a Stein algebra and determine their residue fields, which are $\mathbb{C}$ or a nonstandard complex number field $* \mathbb{C}$

- every maximal ideal in a Stein algebra is the vanishing ideal of a (nonstandard) point.

Another fact which we obtain is a geometric visualisation of generic points in irreducible complex spaces. They are found to be certain nonstandard (but bounded) points. The evaluation map in such a point defines an inclusion

$$
\mathcal{M}(X) \hookrightarrow{ }^{*} \mathbb{C}
$$

of the field $\mathcal{M}(X)$ of meromorphic functions on $X$ to the field $* \mathbb{C}$ of nonstandard complex numbers.

Finally, in the tradition of G.W.Leibniz, we propose a "real" infinitesimal interpretation of the symbol $d x$ in our context and, more generally, the notion of a differential form. Such a $d x$ is an infinitesimal variable; that is (an equivalence class of) a function which maps near standard points to infinitesimal ones. This notion is rigorously defined 
in Section 6. We show that our differential forms on $X$ identify naturally with the holomorphic ones on the associated complex space $X^{a n}$.

In many of our constructions (in fact, in the most interesting ones), we work with socalled external sets (like for instance, the set of infinitesimal internal polynomials). This means in particular that the transfer principle of nonstandard mathematics - its most powerful tool - does not work directly here. But it is still important for intermediate steps in our arguments. The reader should notice that, in contrast to the language used in some books on nonstandard analysis, we always use in this paper the expression "bounded" point instead of a "limited" one and similarly also for other objects, as internal polynomials for example.

The classical text on nonstandard mathematics is Robinson's book [15] and also that of Stroyan and Luxemburg [18]. For a more recent introduction into hyperreals see [8] and for an account to hyper categories [1]. Other interesting rings of nonstandard numbers, motivated by asymptotic expansions, are considered in [13].

\section{Contents}

Introduction $\quad 1$

1 Algebras of internal polynomials 4

1.1 Ultraproduct of rings . . . . . . . . . . . . . . . 4

1.2 Internal algebras . . . . . . . . . . . . . 5

1.3 The ring of internal polynomials $\ldots \ldots \ldots 6$

1.4 The ring of bounded internal polynomials $\ldots \ldots \ldots 7$

2 Comparing holomorphic functions and internal polynomials 11

2.1 Stein Algebras . . . . . . . . . . . . . . . . . . . 11

2.2 Internal holomorphic functions . . . . . . . . . . . . 12

2.3 Internal holomorphic maps into complex hyperbolic spaces . . . . . 14

2.4 Bounded internal holomorphic functions . . . . . . . . . . 17

2.5 The standard part functor in the affine case $\ldots \ldots 20$

2.6 Completions and enlargements . . . . . . . . . . . . . 23 
3 Affine $*$-schemes and *-bounded schemes $\quad 25$

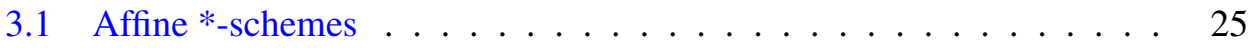

3.2 Affine $*$-bounded schemes . . . . . . . . . . . . . . 31

4 Global *-bounded schemes and the standard part functor 36

4.1 The basic functors . . . . . . . . . . . . . . . 36

4.2 DG- algebra resolutions of complex spaces via bounded polynomial

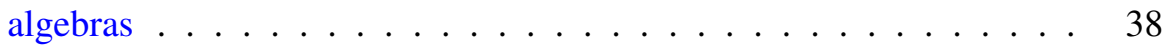

4.3 Special features of the standard part construction $\ldots \ldots$. . . . . 40

5 The Nullstellensatz and nonstandard generic points for complex spaces 41

5.1 Nonstandard zeros of holomorphic functions and the Nullstellensatz . 41

5.2 On the spectrum of a Stein algebra . . . . . . . . . . . . . . 44

6 Differential forms seen in a modern nonstandard way 47

$\begin{array}{lr}\text { Appendix } & \mathbf{5 0}\end{array}$

Bibliography

\section{Algebras of internal polynomials}

Let $I$ be an infinite set and let $\mathcal{U}$ be a fixed non-principal ultrafilter on $I$.

\subsection{Ultraproduct of rings}

Let $\left(A_{i}\right)_{i \in I}$ be a family of sets indexed by $I$. We write ${ }^{*} A_{I}=\Pi_{\mathcal{U}} A_{i}$ for the ultraproduct of the $A_{i}$ 's with respect to the ultrafilter $\mathcal{U}$. An element $x$ of ${ }^{*} A_{I}$ is an equivalence class of $\left(x_{i}\right)$. We write $x=\left[x_{i}\right]$. If $A_{i}=A$ for each $i \in I$, then ${ }^{*} A=\Pi_{\mathcal{U}} A_{i}$ is the ultrapower of $A$ with respect to $\mathcal{U}$. Let $f_{i}: A_{i} \longrightarrow B_{i}$ be a family of maps between $A_{i}$ and $B_{i}$. It induces a map ${ }^{*} f:{ }^{*} A_{I} \longrightarrow{ }^{*} B_{I}$, defined by ${ }^{*} f\left(\left[a_{i}\right]\right)=\left[f_{i}\left(a_{i}\right)\right]$. Such map is called an internal map and we write ${ }^{*} f=\left[f_{i}\right]$.

Journal of Logic \& Analysis 2:9(2010) 
Now, we consider algebraic structures on the $A_{i}$ 's. If $\left(A_{i}\right)_{i}$ is a family of rings, then ${ }^{*} A_{I}$ is again a ring. In fact ${ }^{*} A_{I}=\prod_{i \in I} A_{i} / \mathfrak{I}$, where $\mathfrak{I}$ is the ideal in $\prod_{i \in I} A_{i}$ defined by $\mathfrak{I}=\left\{x \in \Pi_{i \in I} A_{i}, \mathrm{~V}(x) \in \mathcal{U}\right\}$ where $\mathrm{V}(x):=\left\{i \in I \mid x_{i}=0\right\}$. Rings of this form are called $*_{\text {-rings }}$ or internal rings. Let ${ }^{*} A_{I}$ and ${ }^{*} B_{I}$ be two internal rings. An internal homomorphism of ${ }^{*}$-rings between ${ }^{*} A_{I}$ and ${ }^{*} B_{I}$ is given by ${ }^{*} f=\left[f_{i}\right]$ where $f_{i}: A_{i} \longrightarrow B_{i}$ are morphisms of rings. *-rings and internal morphisms of *-rings constitute a category of *-rings. The operator "**" gives a functor between the category of rings, parametrized by the index set $I$, and the category of *-rings. If $A$ is an internal ring, $\left(\mathrm{Alg}_{A}^{\text {int }}\right)$ denotes the category of internal $A$-algebras.

\subsection{Internal algebras}

Let ${ }^{*} S_{I}=\Pi_{\mathcal{U}} S_{i}$ be an internal ring and $n=\left[n_{i}\right] \in{ }^{*} \mathbb{N}$.

The ring of polynomials in indeterminates $X_{1}, \ldots, X_{n_{i}}$ over the ring $S_{i}$ is denoted by $S_{i}\left[X_{1}, \ldots, X_{n_{i}}\right]$ and $S\left[X_{1}, \ldots, X_{n}\right]_{i n t}$ denotes the ultraproduct of the polynomial rings $S_{i}\left[X_{1}, \ldots, X_{n_{i}}\right]$ with respect to the ultrafilter $\mathcal{U}$; that is,

$$
S\left[X_{1}, \ldots, X_{n}\right]_{\text {int }}=\prod_{\mathcal{U}} S_{i}\left[X_{1}, \ldots, X_{n_{i}}\right] .
$$

Given $B \in\left(\operatorname{Alg}_{S}^{i n t}\right)$, we say that $B$ is a *algebra of hyperfinite type over $S$ (resp. *algebra of finite type over $S$ ), if there exist an integer $n \in{ }^{*} \mathbb{N}$ (resp. a finite $n \in \mathbb{N}$ ) and a surjective internal morphism $u: S\left[X_{1}, \ldots, X_{n}\right]_{\text {int }} \longrightarrow B$. Let $\mathfrak{I}=$ $\operatorname{Ker} u$, then $\mathfrak{I}$ is an internal ideal of $S\left[X_{1}, \ldots, X_{n}\right]_{\text {int }}$ and $B$ is internally isomorphic to $S\left[X_{1}, \ldots, X_{n}\right]_{\text {int }} / \mathfrak{I}$. Furthermore, the morphism $u$ is completely determined by the internal sequence $\left(u\left(X_{1}\right), \ldots, u\left(X_{n}\right)\right)$. Conversely, any hyperfinite family $\left(t_{1}, \ldots, t_{n}\right) \in$ $B^{n}$ determines a unique internal morphism $u: S\left[X_{1}, \ldots, X_{n}\right]_{\text {int }} \longrightarrow B$ such that $u\left(X_{i}\right)=$ $t_{i}$ for each $i=1, \ldots, n$.

If the ring ${ }^{*} S_{I}$ is $*$-noetherian (that is, $\left\{i \in I, S_{i}\right.$ is noetherian $\} \in \mathcal{U}$ ), then the ideal $\mathfrak{I}$ is hyperfinitely generated by a family of nonstandard polynomials.

Let $B$ and $C$ be two *-algebras of hyperfinite type over ${ }^{*} S_{I}$. Then there exist $n, m \in$ ${ }^{*} \mathbb{N}$ and surjective morphisms $u: S\left[X_{1}, \ldots, X_{n}\right]_{\text {int }} \longrightarrow B, v: S\left[Y_{1}, \ldots, Y_{m}\right]_{\text {int }} \longrightarrow C$, such that we have internal isomorphisms $B \simeq S\left[X_{1}, \ldots, X_{n}\right]_{\text {int }} / \mathfrak{I}, C \simeq S\left[Y_{1}, \ldots, Y_{m}\right]_{\text {int }} / \mathfrak{J}$.

Let $w: B \longrightarrow C$ be a morphism of *-algebras. Then the morphism $w$ lifts to a morphism of $*$-algebras $\tilde{w}: S\left[X_{1}, \ldots, X_{n}\right]_{\text {int }} \longrightarrow S\left[Y_{1}, \ldots, Y_{m}\right]_{\text {int }}$, sending the ideal $\mathfrak{I}$ into the ideal $\mathfrak{J}$.

In the sequel, let $n$ be a fixed finite integer. 


\subsection{The ring of internal polynomials}

Let ${ }^{*} S_{I}=\Pi_{\mathcal{U}} S_{i}$ be an internal ring. Elements of $S\left[X_{1}, \ldots, X_{n}\right]_{\text {int }}$ are called internal polynomials or nonstandard polynomials. Suppose now we are given $d \in{ }^{*} \mathbb{N}$. An internal polynomial $P \in S\left[X_{1}, \ldots, X_{n}\right]_{i n t}$ of degree at most $d$, will be written in the unique form

$$
P=\sum_{|\nu| \leq d} a_{\nu} X^{\nu}, \text { where } a_{\nu} \in{ }^{*} S_{I} \text { and } d \in{ }^{*} \mathbb{N} \text {. }
$$

Here the sum ranges over all multi-indices $\nu=\left(\nu_{1}, \ldots, \nu_{n}\right) \in{ }^{*} \mathbb{N}^{n}$ with $|\nu|=$ $\nu_{1}+\ldots+\nu_{n} \leq d$ and as usual $X^{\nu}$ is stands for $X_{1}^{\nu_{1}} \ldots X_{n}^{\nu_{n}}$. We have a canonical injective morphism of $\left({ }^{*} S_{I}\right)$-algebra $\left({ }^{*} S_{I}\right)\left[X_{1}, \ldots, X_{n}\right] \longrightarrow S\left[X_{1}, \ldots, X_{n}\right]_{\text {int }}$, where $\left({ }^{*} S_{I}\right)\left[X_{1}, \ldots, X_{n}\right]$ denotes the ring of polynomials in indeterminates $X_{1}, \ldots, X_{n}$ over the internal ring ${ }^{*} S_{I}$. Furthermore, let $P \in S\left[X_{1}, \ldots, X_{n}\right]_{\text {int }}$ be an internal polynomial of degree $d$, then $P \in\left({ }^{*} S_{I}\right)\left[X_{1}, \ldots, X_{n}\right]$ if and only if, $d$ is finite.

We denote by $\left({ }^{*} S_{I}\right) \llbracket X_{1}, \ldots, X_{n} \rrbracket$ the ring of power series over the internal ring ${ }^{*} S_{I}$ in indeterminates $X_{1}, \ldots, X_{n}$.

Proposition 1.1 There is a canonical surjective ring homomorphism

$$
\theta: S\left[X_{1}, \ldots, X_{n}\right]_{i n t} \longrightarrow\left({ }^{*} S_{I}\right) \llbracket X, \ldots, X_{n} \rrbracket
$$

given by "forgetting monomials of infinite degree".

Proof Let $P \in S\left[X_{1}, \ldots, X_{n}\right]_{i n t}$. Then $P=\sum_{|\nu| \leq d} a_{\nu} X^{\nu}$, where $a_{\nu} \in{ }^{*} S_{I}$ and $d \in$ $* \mathbb{N}$. Let $\theta$ be the restriction map

$$
\theta(P)=\sum_{\nu \in \mathbb{N}^{n}} a_{\nu} X^{\nu} \in\left({ }^{*} S_{I}\right) \llbracket X, \ldots, X_{n} \rrbracket ;
$$

that is, $\theta(P)$ is the standard power series with coefficients in ${ }^{*} S_{I}$. It is clear that the map $\theta$ is a ring homomorphism and $\theta$ maps onto $\left({ }^{*} S_{I}\right) \llbracket X, \ldots, X_{n} \rrbracket$. In fact, given $Q \in\left({ }^{*} S_{I}\right) \llbracket X, \ldots, X_{n} \rrbracket, Q=\Sigma_{\nu \in \mathbb{N}^{n}} a_{\nu} X^{\nu}$, by comprehensiveness (see for example [13] Ch.2§ 6), the sequence $\left(a_{\nu}\right)_{\nu \in \mathbb{N}^{n}}$ extends to an internal sequence, also denoted by $\left(a_{\nu}\right)_{\nu \in \mathbb{N}^{n}}$. Fix any $d \in{ }^{*} \mathbb{N}_{\infty}$ and put $P=\sum_{|\nu| \leq d} a_{\nu} X^{\nu}$. Then clearly we have $\theta(P)=Q$. - 


\subsection{The ring of bounded internal polynomials}

Let ${ }^{*} \mathbb{C}$ be an enlargement of the field of complex numbers. Elements of the ring $\mathbb{C}\left[X_{1}, \ldots, X_{n}\right]_{\text {int }}$ can be considered as internal functions between ${ }^{*} \mathbb{C}^{n}$ and ${ }^{*} \mathbb{C}$. In the sequel, we will not distinguish between internal polynomials and their associated internal functions. As mentioned above, each $P \in \mathbb{C}\left[X_{1}, \ldots, X_{n}\right]_{\text {int }}$ can be written in the form $P=\Sigma_{|\nu| \leq d} a_{\nu} X^{\nu}$, where $a_{\nu} \in{ }^{*} \mathbb{C}$ and $d \in{ }^{*} \mathbb{N}$. We associate to $P$ the internal polynomial $|P|$ defined by

$$
|P|:=\sum_{|\nu| \leq d}\left|a_{\nu}\right| X^{\nu}
$$

where $|a|$ is the (nonstandard) absolute value of $a \in{ }^{*} \mathbb{C}$.

Let us fix some notations: ${ }^{b} \mathbb{C}$ stands for the set of bounded points of $* \mathbb{C}$ and ${ }^{i} \mathbb{C}$ for the set of infinitesimal points of ${ }^{*} \mathbb{C}$. We denote by ${ }^{*} \mathbb{N}_{\infty}$ the set of infinite integers. If $n, m \in{ }^{*} \mathbb{N}$ with $n \leq m, \llbracket n \ldots m \rrbracket:=\left\{k \in{ }^{*} \mathbb{N}, n \leq k \leq m\right\}$. It is evident that ${ }^{b}\left(\mathbb{C}^{n}\right)=\left({ }^{b} \mathbb{C}\right)^{n}$ and ${ }^{i}\left(\mathbb{C}^{n}\right)=\left({ }^{i} \mathbb{C}\right)^{n}$.

Definition 1.2 Let $P \in \mathbb{C}\left[X_{1}, \ldots, X_{n}\right]_{\text {int }}$ be an internal polynomial. We call $P$

i) a bounded polynomial if $P\left({ }^{b} \mathbb{C}^{n}\right) \subset{ }^{b} \mathbb{C}$, that is, $P$ sends bounded points of ${ }^{*} \mathbb{C}^{\text {n }}$ to bounded points of $* \mathbb{C}$,

ii) an absolutely bounded polynomial if $|P|\left({ }^{b} \mathbb{C}^{n}\right) \subset{ }^{b} \mathbb{C}$, that is, $|P|$ sends bounded points of $* \mathbb{C}^{\mathrm{n}}$ to bounded points of $* \mathbb{C}$,

iii) an infinitesimal polynomial (resp. absolutely infinitesimal polynomial) if $P\left({ }^{b} \mathbb{C}^{n}\right) \subset$ ${ }^{i} \mathbb{C}$ (resp. $\left.|P|\left({ }^{b} \mathbb{C}^{n}\right) \subset{ }^{i} \mathbb{C}\right)$

In fact, we will prove in 1.5 that these two notions of boundedness defined above coincide.

Let ${ }^{b} \mathbb{C}\left[X_{1}, \ldots, X_{n}\right]$ denote the set of bounded internal polynomials. It is a subring of $\mathbb{C}\left[X_{1}, \ldots, X_{n}\right]_{\text {int }}$. The subset ${ }^{i} \mathbb{C}\left[X_{1}, \ldots, X_{n}\right]$ of infinitesimal internal polynomials is an ideal of ${ }^{b} \mathbb{C}\left[X_{1}, \ldots X_{n}\right]$.

NotAtion. Let ${ }^{b s} \mathbb{C}\left[X_{1}, \ldots, X_{n}\right]:=\left\{P=\Sigma a_{\nu} X^{\nu} \in{ }^{b} \mathbb{C}\left[X_{1}, \ldots, X_{n}\right] \mid a_{\nu} \in\right.$ $\mathbb{C}$ for every standard $\nu\}$. Trivially it is a subring of ${ }^{b} \mathbb{C}\left[X_{1}, \ldots, X_{n}\right]$.

If the degree of an internal polynomial is finite, we can immediately characterize bounded or infinitesimal polynomials by their coefficients and the notions of boundedness and absolutely boundedness coincide. 
Remark Let $P=\Sigma_{|\nu| \leq d} a_{\nu} X^{\nu}$ be an internal polynomial of bounded degree $d$, where $a_{\nu} \in{ }^{*} \mathbb{C}$. Then

i) $P$ is a bounded polynomial if and only if $a_{\nu} \in{ }^{b} \mathbb{C}$ for each $\nu \in \mathbb{N}^{n}$, such that $|\nu| \leq d$,

ii) $P$ is an infinitesimal polynomial if and only if $a_{\nu} \in{ }^{i} \mathbb{C}$ for each $\nu \in \mathbb{N}^{n}$, such that $|\nu| \leq d$.

We have the inclusions

$$
\left({ }^{b} \mathbb{C}\right)\left[X_{1}, \ldots, X_{n}\right] \subsetneq^{b} \mathbb{C}\left[X_{1}, \ldots, X_{n}\right] \subsetneq \mathbb{C}\left[X_{1}, \ldots, X_{n}\right]_{\text {int }} .
$$

Now, we consider the general case

Proposition 1.3 Let $P \in \mathbb{C}\left[X_{1}, \ldots, X_{n}\right]_{\text {int }}$ be an internal polynomial; that is

$$
P=\sum_{|\nu| \leq d} a_{\nu} X^{\nu}, \text { where } a_{\nu} \in{ }^{*} \mathbb{C} \text { and } d \in{ }^{*} \mathbb{N}_{\infty}
$$

Then $P$ is absolutely bounded if and only if the following two conditions are satisfied

i) $a_{\nu} \in{ }^{b} \mathbb{C}$ for bounded $|\nu|$ (that is, $\nu \in \mathbb{N}^{n}$ ),

ii) $\left|a_{\nu}\right|^{\frac{1}{|\nu|}} \in{ }^{i} \mathbb{C}$ for infinite $|\nu|$ such that $|\nu| \leq d$.

Proof Let $P=\Sigma_{|\nu| \leq d} a_{\nu} X^{\nu}$ be an absolutely bounded internal polynomial, then for each $\nu \in \mathbb{N}^{n}$, we have $\left|a_{\nu}\right| \leq|P|(1, \ldots, 1)$ so we conclude that $a_{\nu} \in{ }^{b} \mathbb{C}$ for finite $|\nu|$. Suppose that there exists a standard positive real $m$, such that

$$
\left|a_{\nu}\right|^{\frac{1}{|\nu|}} \geq m \text { for some infinite }|\nu| \text { such that }|\nu| \leq d .
$$

Let $q$ be a standard real such that $q>1$ and $\xi$ be a standard positive real such that $\xi>\frac{q}{m}$. Then,

$$
|P|(\xi, \ldots, \xi) \geq\left|a_{\nu}\right| \xi^{|\nu|} \geq(m \xi)^{|\nu|} \geq q^{|\nu|}
$$

hence, $|P|(\xi, \ldots, \xi)$ will be infinite, which is a contradiction.

Now, let $\varepsilon>0$ be a standard positive real. We set

$$
A_{\varepsilon}=\left\{|\nu| \in{ }^{*} \mathbb{N},\left|a_{\nu}\right|^{\frac{1}{|\nu|}} \leq \varepsilon\right\} .
$$


Then $A_{\varepsilon}$ is an internal subset of ${ }^{*} \mathbb{N}$ which contains $\left\{n \in{ }^{*} \mathbb{N}_{\infty}, n \leq d\right\}$. By the permanence principle, there exists a finite integer $n_{0} \in \mathbb{N}$, such that $\llbracket n_{0} . . d \rrbracket \subset A_{\varepsilon}$. This means that,

$$
\left|a_{\nu}\right|^{\frac{1}{|\nu|}} \leq \varepsilon \text { for every } \nu \in{ }^{*} \mathbb{N}^{n}:|\nu| \in \llbracket n_{0} . . d \rrbracket .
$$

Let $x=\left(x_{1}, \ldots, x_{n}\right)$ be a bounded point in ${ }^{*} \mathbb{C}^{n}$, such that $\left|x_{i}\right| \leq \frac{1}{2 \varepsilon}$ for each $i \in$ $\llbracket 1 \ldots n \rrbracket$. Then, we have

$$
\left|a_{\nu} x^{\nu}\right| \leq \varepsilon^{|\nu|}\left|x^{\nu}\right| \leq\left(\frac{1}{2}\right)^{|\nu|} \text { for each } \nu \in{ }^{*} \mathbb{N}^{n}:|\nu| \in \llbracket n_{0} . . d \rrbracket .
$$

The two internal polynomials $\Sigma_{n_{0} \leq|\nu| \leq d}\left|a_{\nu}\right| X^{\nu}$ and $\Sigma_{|\nu|<n_{0}}\left|a_{\nu}\right| X^{\nu}$ are bounded for each $x=\left(x_{1}, \ldots, x_{n}\right)$, such that $\left|x_{i}\right| \leq \frac{1}{2 \varepsilon}$.

Now, let $x=\left(x_{1}, \ldots, x_{n}\right)$ be a bounded point in ${ }^{*} \mathbb{C}^{n}$. Then there exists a standard positive real $\varepsilon$ such that $\left|x_{i}\right| \leq \frac{1}{2 \varepsilon}$ for each $i \in \llbracket 1 \ldots n \rrbracket$. Hence $|P|\left(x_{1}, \ldots x_{n}\right)$ is bounded.

Proposition 1.4 Let $P \in \mathbb{C}\left[X_{1}, \ldots, X_{n}\right]_{\text {int }}$ be an internal polynomial, so

$$
P=\sum_{|\nu| \leq d} a_{\nu} X^{\nu} \text { where } a_{\nu} \in{ }^{*} \mathbb{C} \text { and } d \in{ }^{*} \mathbb{N}_{\infty}
$$

Then $P$ is absolutely infinitesimal if and only if $\left|a_{\nu}\right|^{\frac{1}{|\nu|}} \in{ }^{i} \mathbb{C}$ for each $\nu \in{ }^{*} \mathbb{N}^{n} \backslash\{0\}$, $|\nu| \leq d$, and $a_{0}$ infinitesimal.

The last condition is equivalent to $a_{\nu} \in{ }^{i} \mathbb{C}$, if $|\nu|$ is finite (that is, $\nu \in \mathbb{N}^{n}$ ) and $\left|a_{\nu}\right|^{\frac{1}{|\nu|}} \in{ }^{i} \mathbb{C}$, if $|\nu| \leq d$ and $|\nu|$ is infinite.

Proof Let us define

$$
A:=\left\{\left|a_{\nu}\right|^{\frac{1}{\nu 1}}, 0<|\nu| \leq d\right\} .
$$

Then $A$ is an hyperfinite set and therefore has a greatest element. Let $M=\max A$, so $M \in A$ and $M \in{ }^{i} \mathbb{C}$.

Let $x=\left(x_{1}, \ldots, x_{n}\right)$ be a bounded point of ${ }^{*} \mathbb{C}^{n}$. Then there exists a standard positive real $R>0$ such that $\left|x_{i}\right| \leq R$ for each $i \in \llbracket 1 \ldots n \rrbracket$. We get

$$
|| P\left|\left(x_{1}, \ldots, x_{n}\right)\right| \leq\left|a_{0}\right|+\sum_{1 \leq|\nu| \leq d}(M R)^{|\nu|} \leq\left|a_{0}\right|+\frac{M R}{(1-M R)^{n}} \in{ }^{i} \mathbb{C} .
$$

In order to show the other implication, we note that the internal polynomial $P$ is in particular absolutely bounded, so by Proposition 1.3 , we have $\left|a_{\nu}\right|^{\frac{1}{\nu \mid}} \in{ }^{i} \mathbb{C}$ for infinite $|\nu|$. For each $\nu \in \mathbb{N}^{n}$, we have $\left|a_{\nu}\right| \leq|P|(1, \ldots, 1)$. Then, we conclude that $a_{\nu} \in{ }^{i} \mathbb{C}$ for finite $|\nu|$. 
Proposition 1.5 Let $P \in \mathbb{C}\left[X_{1}, \ldots, X_{n}\right]_{\text {int }}$ be an internal polynomial. We have

i) $P$ is a bounded polynomial if and only if $P$ is an absolutely bounded polynomial,

ii) $P$ is an infinitesimal polynomial if and only if $P$ is an absolutely infinitesimal polynomial.

Proof Let $x=\left(x_{1}, \ldots, x_{n}\right) \in{ }^{*} \mathbb{C}^{n}$, we have $\left|P\left(x_{1}, \ldots, x_{n}\right)\right| \leq|P|\left(\left|x_{1}\right|, \ldots,\left|x_{n}\right|\right)$. So, it is clear that every absolutely bounded (resp. infinitesimal) polynomial is a bounded (resp. an infinitesimal) polynomial. Now we verify the converse. Let $P=$ $\Sigma_{|\nu| \leq d} a_{\nu} X^{\nu}$ be a bounded polynomial and $R$ be a standard positive real. By Proposition 1.3, we have to prove that $a_{\nu}$ is bounded for finite $|\nu|$ and $\left|a_{\nu}\right|^{\frac{1}{|\nu|}}$ is infinitesimal for infinite $|\nu|$. We denote by $T_{R}=\left\{\left(\xi_{1}, \ldots \xi_{n}\right) \in{ }^{*} \mathbb{C}^{n},\left|\xi_{1}\right|=\ldots=\left|\xi_{n}\right|=R\right\}$. Applying transfer to the Cauchy formula, we obtain

$$
a_{\nu}=\frac{1}{(2 \pi i)^{n}} \int_{T_{R}} \frac{P\left(\xi_{1}, \ldots, \xi_{n}\right)}{\xi_{1}^{\nu_{1}+1} \ldots \xi_{n}^{\nu_{n}+1}} d \xi_{1} \ldots d \xi_{n}
$$

Again, by transfer, the polynomial $P$ attains its maximum on the Shilov boundary of the polydisc at some point $\xi_{R} \in T_{R}$. But since $P$ is bounded, we have $\left|P\left(\xi_{R}\right)\right|$ a bounded number. Hence, there exists a standard positive real $M_{R}$ such that

$$
\left|a_{\nu}\right| \leq \frac{M_{R}}{R^{|\nu|}}, \forall \nu \in{ }^{*} \mathbb{N}^{n},|\nu| \leq d
$$

So, if $|\nu|$ is bounded then $a_{\nu}$ is bounded and if $|\nu|$ is infinite, then $M_{R}^{\frac{1}{|\nu|}} \approx 0$ and we have

$$
\left|a_{\nu}\right|^{\frac{1}{|\nu|}} \leq \frac{2}{R} \text { for each } \nu \text { such that }|\nu| \in{ }^{*} \mathbb{N}_{\infty},|\nu| \leq d
$$

Since $R$ is an arbitrary standard positive real, we get $\left|a_{\nu}\right|^{\frac{1}{|\nu|}} \in{ }^{i} \mathbb{C}$ for infinite $|\nu|$.

Corollary 1.6 The rings ${ }^{b} \mathbb{C}\left[X_{1}, \ldots, X_{n}\right]$ and ${ }^{i} \mathbb{C}\left[X_{1}, \ldots, X_{n}\right]$ are invariant by any partial derivative $\partial^{\alpha}$ for $\alpha \in \mathbb{N}^{n}$.

Remark There is a more general version of the preceding results (including Cauchy's formula for the coefficients), assuming only that the given internal polynomial takes bounded/infinitesimal values on a polydisc with appreciable multiradius. 


\section{Comparing holomorphic functions and internal polynomi- als}

We have already defined the ring of bounded internal polynomials over $* \mathbb{C}$. Let $P$ be a bounded internal polynomial. Then $P$ can be seen as an internal function from the set of bounded points of ${ }^{*} \mathbb{C}^{n}$ to the set of bounded points of ${ }^{*} \mathbb{C}$. First, we prove that ${ }^{\circ} P$, the standard part of $P$, is an entire holomorphic function on $\mathbb{C}^{n}$. This result is an easy corollary of the theorem of Robinson-Callot which we extended to several complex variables.

In this section, we define the so-called standard part functor from the category of bounded polynomial algebras to the category of Stein algebras of finite embedding dimension. We prove that this functor is essentially surjective. This fact can be regarded as a nonstandard algebraization of Stein spaces.

\subsection{Stein Algebras}

We recall some known facts about Stein algebras. Let $\left(X, \mathcal{O}_{X}\right)$ be a complex space. Then $X$ is called Stein space, if it is holomorphically separable and holomorphically convex. The algebra $\Gamma\left(X, \mathcal{O}_{X}\right)$ of all holomorphic functions on $X$ is a Fréchet algebra. A topological algebra $A$ (over $\mathbb{C}$ ) is called a Stein algebra, if there exists a Stein space $\left(X, \mathcal{O}_{X}\right)$ such that $A$ is morphically isomorphic to $\Gamma\left(X, \mathcal{O}_{X}\right)$.

Stein algebras form a category where morphisms are morphisms of topological $\mathbb{C}$ algebras. The functor of global sections defines a contravariant functor from the category of Stein spaces to the category of Stein algebras. In fact, this functor is an anti-equivalence between these categories, see Forster[6] for the algebro-topological theory of Stein algebras.

We recall some results due to H.Cartan. Let $A=\Gamma\left(X, \mathcal{O}_{X}\right)$ be a Stein algebra, and $\mathfrak{a}$ is a closed ideal of $A$. Then $\mathcal{O}_{X} \cdot \mathfrak{a}$ is a coherent sheaf of ideals and $\Gamma\left(X, \mathcal{O}_{X} \cdot \mathfrak{a}\right)$ is isomorphic to $\mathfrak{a}$. Conversely, let $\mathcal{M}$ be a coherent sheaf of ideals of $\mathcal{O}_{X}$. Then $\Gamma(X, \mathcal{M})$ is a closed ideal of $A$ and $\mathcal{O}_{X} \cdot \Gamma(X, \mathcal{M})$ identifies with $\mathcal{M}$.

Let $A$ be a Stein algebra of finite embedding dimension. Then $A=\Gamma\left(X, \mathcal{O}_{X}\right)$, where $X$ is Stein space of finite embedding dimension. It follows from the proper embedding theorem that $X$ can be embedded as a closed complex subspace of $\mathbb{C}^{n}$ for some $n \in \mathbb{N}$. 


\subsection{Internal holomorphic functions}

By the enlargement construction, one can define the set of internal holomorphic functions on an internal open subset of ${ }^{*} \mathbb{C}$. Robinson studied internal holomorphic functions and proved some external properties of these functions.

Let $\mathcal{U}$ be an ultrafilter defined on $\mathbb{N}$ and let ${ }^{*} \mathbb{C}=\Pi_{\mathcal{U}} \mathbb{C}$ be an enlargement of $\mathbb{C}$. Let $D=\left[D_{i}\right]$ be an internal subset of ${ }^{*} \mathbb{C}^{n}$ and let $f=\left[f_{i}\right]: D \longrightarrow^{*} \mathbb{C}$ be an internal function. We say that $f$ is an internal holomorphic function on $D$ if

$$
\left\{i \in \mathbb{N} \mid D_{i} \subset \mathbb{C}^{n} \text { open and } f_{i} \text { holomorphic on } D_{i}\right\} \in \mathcal{U} \text {. }
$$

It is well known, by Osgood's theorem, that a function $f$ is holomorphic on $\Omega$, an open subset of $\mathbb{C}^{n}$, if and only if $f$ is continuous on $\Omega$ and partially holomorphic on $\Omega$.

Theorem 2.1 Let $B$ be an $S$-open subset of ${ }^{*} \mathbb{C}^{n}$. We fix a bounded point $a$ in $B$ and $f: B \longrightarrow{ }^{*} \mathbb{C}$ an internal holomorphic function on $B$. We assume that $f$ is bounded on $\mu(a)$, the halo of $a$. Then there exists $V$ an $S$-open neighbourhood of $a$ such that

i) $f$ is $S$-continuous in $V$.

ii) There exists ${ }^{\circ} f:{ }^{\circ} V \longrightarrow \mathbb{C}$ a holomorphic function in ${ }^{\circ} V$, such that ${ }^{\circ} f \approx f$ and ${ }^{\circ}\left(\partial^{\alpha} f\right)=\partial^{\alpha}\left({ }^{\circ} f\right)$ for each $\alpha \in \mathbb{N}^{n}$.

iii) If ${ }^{\circ} f$ is not constant in ${ }^{\circ} V$, then $f$ is $S$-open in $a$, that is, $f(\mu(a))=\mu(f(a))$.

This theorem is an easy generalization of the theorem of Robinson-Callot known in single complex variables, see Robinson[15], Callot[3], Fruchard[7] and Lutz-Goze([14]: lesson 11, p:123).

Notation. For $\nu=\left(\nu_{1}, \ldots, \nu_{n}\right) \in \mathbb{N}^{n}$ and $z=\left(z_{1}, \ldots, z_{n}\right) \in \mathbb{C}^{n}$ define $|\nu|=\sum_{i=1}^{n} \nu_{i}$ and $z^{\nu}=z_{1}^{\nu_{1}} \ldots z_{n}^{\nu_{n}}$. Let $r=\left(r_{1}, \ldots, r_{n}\right) \in{ }^{*} \mathbb{R}^{n}$, all $r_{i}>0, z_{0}=\left(z_{1}^{(0)}, \ldots, z_{n}^{(0)}\right) \in$ $* \mathbb{C}^{n}$. Then $\mathrm{P}^{n}\left(z_{0}, r\right)=\left\{z \in \mathbb{C}^{n}|| z_{i}-z_{i}^{(0)} \mid<r_{i}\right.$ for $\left.i=1, \ldots, n\right\}$ is called the *open polydisk with polyradius $r$ and center $z_{0}$ and $\overline{\mathrm{P}^{n}(a, r)}=\left\{z \in{ }^{*} \mathbb{C}^{n}|| z_{i}-z_{i}^{(0)} \mid \leq\right.$

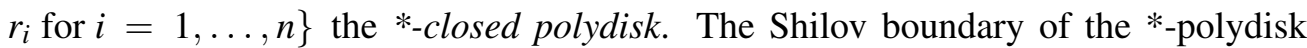
$\mathrm{P}^{n}\left(z_{0}, r\right)$ is the set $\mathrm{T}^{n}\left(z_{0}, r\right)=\left\{z \in \mathbb{C}^{n}|| z_{i}-z_{i}^{(0)} \mid=r_{i}\right.$ for $\left.i=1, \ldots, n\right\}$. If $r>0$ and $r=(r, \ldots, r)$, we write $\mathrm{P}_{r}^{n}\left(z_{0}\right)\left(\operatorname{resp} . \mathrm{T}_{r}^{n}\left(z_{0}\right)\right)$ instead of $\mathrm{P}^{n}\left(z_{0}, r\right)\left(\operatorname{resp} . \mathrm{T}^{n}\left(z_{0}, r\right)\right)$.

Proof i) By the permanence principle, there exists a standard positive real $r$ such that $\overline{\mathrm{P}^{n}(a, r)} \subset B$ and the internal holomorphic function $f$ is bounded on $\overline{\mathrm{P}^{n}(a, r)}$. Let $\rho$ be a standard positive such that $\rho<r$ and

$$
V:=\left\{z \in{ }^{*} \mathbb{C}^{n}\left|{ }^{\circ}\right| z_{i}-a_{i} \mid<\rho \text { for } i=1, \ldots, n\right\} .
$$


$V$ is an S-open neighbourhood of $a$ and ${ }^{\circ} V$ is the open polydisk with polyradius $\rho$ and center ${ }^{\circ} a$. Let $z, \xi \in V$ such that $z \approx \xi$. Applying transfer to Cauchy's formula, we have

$$
f(z)=\frac{1}{2 \pi i} \int_{\mathrm{T}_{r}^{n}(a)} \frac{f(w)}{w-z} d w \text { and } f(\xi)=\frac{1}{2 \pi i} \int_{\mathrm{T}_{r}^{n}(a)} \frac{f(w)}{w-\xi} d w
$$

where $w-z=\left(w_{1}-z_{1}\right) \ldots\left(w_{n}-z_{n}\right)$ and $w-\xi=\left(w_{1}-\xi_{1}\right) \ldots\left(w_{n}-\xi_{n}\right)$.

Again, by transfer, the function $f$ attains its maximum on $\mathrm{T}_{r}^{n}(a)$, the Shilov boundary of the polydisk $\mathrm{P}^{n}(a, r)$. Since $f$ is bounded on $\overline{\mathrm{P}^{n}(a, r)}$, then $M:=\sup _{w \in \mathrm{T}^{n}(a, r)}|f(w)|$ is bounded. So,

$$
|f(z)-f(\xi)| \leq \frac{M}{(2 \pi)^{n}} \int_{\mathrm{T}_{r}^{n}(a)}\left|\frac{1}{w-z}-\frac{1}{w-\xi}\right| d w .
$$

It is easy to see that $w-z \approx w-\xi$ and, since $w-\xi$ is appreciable, $\frac{1}{w-z} \approx \frac{1}{w-\xi}$. Moreover, there exists $\eta$ a positive infinitesimal such that $\left|\frac{1}{w-z}-\frac{1}{w-\xi}\right| \leq \eta$ for every $w \in \mathrm{T}_{r}^{n}(a)$. It follows that

$$
|f(z)-f(\xi)| \leq M r^{n} \eta
$$

This shows that $f(z) \approx f(\xi)$ for $z \approx \xi$ which is equivalent to S-continuity of $f$ in $z$, see Appendix C Nonstandard Topologies.

ii) Let ${ }^{\circ} a=\left({ }^{\circ} a_{1}, \ldots,{ }^{\circ} a_{n}\right)$ be the standard part of $a$. The standard part ${ }^{\circ} f$ of the function $f$ exist on ${ }^{\circ} V$, the standard polydisk with polyradius $\rho$ and center ${ }^{\circ} a$. We deduce from the first assertion that ${ }^{\circ} f$ is continuous on ${ }^{\circ} V$ (see Appendix C Nonstandard Topologies). By Osgood's theorem, it suffices to prove that $f$ is partially holomorphic. But this follows directly from Robinson [15]( Theorem 6.2.3 p. 156) and for each $\alpha \in \mathbb{N}^{n}$, we have ${ }^{\circ}\left(\partial^{\alpha} f\right)=\partial^{\alpha}\left({ }^{\circ} f\right)$.

iii) The S-continuity of $f$ implies that $f(\mu(a)) \subset \mu(f(a))$. Let $w \approx f(a)$. By hypothesis, ${ }^{\circ} f$, the standard part of $f$, is not constant, hence, there exists $b \neq a$, such that ${ }^{\circ} f(b) \neq$ ${ }^{\circ} f(a)$. Let $D$ be the *-complex line passing through $a$ and $b$. Let $f_{1}$ denote the restriction of $f$ on $D \cap \mu(a)$. $f_{1}$ is an internal holomorphic map and not a constant function. Then, by Robinson's theorem [15] Theorem 6.2.8 p. 158, there exists $z \in$ $D \cap \mu(a)$ such that $f(z)=w$.

Remark The condition that ${ }^{\circ} f$ is not constant is essential to deduce that $f$ is S-open. For a counterexample, it suffices to consider the trivial example of constant infinitesimal maps. Moreover, this condition cannot be weakened by " $f$ is non constant": Let $\varepsilon$ be an infinitesimal and $f(z)=\varepsilon z$. Then we have $f(\mu(0)) \subsetneq \mu(0)$. 


\subsection{Internal holomorphic maps into complex hyperbolic spaces}

In Theorem 2.1, we note that the condition that $f$ is bounded in every point of $\mu(a)$, the halo of $a$, is essential and cannot be replaced by the condition that $f(a)$ is bounded as this example shows. Let $\omega$ be an infinite real and $f(z)=\omega z$ be the internal holomorphic function on ${ }^{*} \mathbb{C}$. We have $f(0)=0$ but $f$ is not bounded on every point of $\mu(0)$. Hence, we could think of imposing additional assumptions on the target space, but keeping a weak condition on $f$. In this direction A. Robinson proved (see [15] Theorem 6.3.2 p.160)

Theorem 2.2 [15] Let $B$ be an $S$-domain in ${ }^{*} \mathbb{C}$ and $f: B \rightarrow{ }^{*} \mathbb{C} \backslash\{0,1\}$ be an internal holomorphic function. Suppose that $f$ takes a bounded value at some point $z_{0} \in B$. Then $f$ is bounded and $S$-continuous in $B$.

We should note that this theorem is not true if $f$ takes values in ${ }^{*} \mathbb{C} \backslash\{0\}$ as the following example shows: put $f(z):=\exp \omega z$, where $\omega$ is an infinite real; $f(0)$ is bounded, but $f$ is not $S$-continuous in 0 .

We want to generalize the above theorem of Robinson, motivated by the fact that the space $\mathbb{C} \backslash\{0,1\}$ is complete hyperbolic and, moreover, hyperbolically embedded in $\mathbb{P}_{\mathbb{C}}^{1}$.

NotAtion. $\Delta_{r}$ stands for the disc with radius $r>0$ in the complex plane $\mathbb{C}$. If $r=1$, we simply denote $\Delta$ instead of $\Delta_{1}$. Let $X$ be a reduced complex space and $\operatorname{Hol}(\Delta, X)$ the set of all holomorphic maps from $\Delta$ to $X$. We will denote by $d_{X}$ the Kobayashi pseudo-distance of $X$.

Theorem 2.3 Let $X$ be a hyperbolic complex space and $f:{ }^{*} \Delta \rightarrow{ }^{*} X$ be an internal holomorphic map. Then

i) $f$ is $S$-continuous in ${ }^{*} \Delta$.

ii) Suppose that $f$ takes a bounded value at some point $z_{0}$ such ${ }^{\circ}\left|z_{0}\right|<1$. Then $f$ is bounded in every point of $\left\{\left.z \in^{*} \Delta\right|^{\circ}|z|<1\right\}$, the $S$-interior of ${ }^{*} \Delta$.

For the definition of bounded points in a metric space, see Appendix C: Metric spaces

Proof For every $g \in \operatorname{Hol}(\Delta, X)$, we have $d_{X}(g(x), g(y)) \leq d_{\Delta}(x, y)$ for any $x, y \in \Delta$. Hence by transfer, we obtain

$$
{ }^{*} d_{X}(f(x), f(y)) \leq{ }^{*} d_{\Delta}(x, y) \text { for each } x, y \in{ }^{*} \Delta .
$$


As a consequence, if $x, y \in{ }^{*} \Delta$ such that $x \approx y$, then ${ }^{*} d_{X}(f(x), f(y)) \approx 0$ and $f$ is S-continuous on ${ }^{*} \Delta$. The assertion (ii) is immediately deduced from the following lemma, due to Robinson (see [15], Theorem 4.5.9 p.114)

Lemma 2.4 [15] Let $h$ be an $S$-continuous map, defined on an $S$-connected set $D$. Then the points of $h(D)$ belong to the same galaxy.

Next, we consider the case of complete hyperbolic complex spaces.

Theorem 2.5 Let $f:{ }^{*} \Delta \rightarrow{ }^{*} X$ be an internal holomorphic map where $X$ is a complete hyperbolic complex space. Then

i) $f$ is $S$-continuous in ${ }^{*} \Delta$.

ii) Suppose that $f$ takes a near-standard value at some point $z_{0}$ such ${ }^{\circ}\left|z_{0}\right|<1$. Then $f$ is near-standard in every point of $\left\{\left.z \in{ }^{*} \Delta\right|^{\circ}|z|<1\right\}$, the $S$-interior of ${ }^{*} \Delta$ and there exists a holomorphic map ${ }^{\circ} f: \Delta \rightarrow X$, the standard part of $f$ verifying ${ }^{\circ} f \approx f$.

We recall the following definition

Definition 2.6 Let $(X, d)$ be a metric space. We say that $(X, d)$ is strongly complete or finitely compact if every closed ball $B(x, r)=\{y \in X \mid d(x, y) \leq r\}$ with $x \in X$ and $r>0$ is compact.

Comparing different notions of completeness, Kobayashi proved (see [11], Proposition 1.1 .9 p.4)

Proposition 2.7 [11] Let $d$ be a distance on a locally compact space $X$. Then

i) If $(X, d)$ is strongly complete then $(X, d)$ is complete.

ii) If $d$ is inner distance then completeness implies strong completeness.

It is straightforward, using a nonstandard characterization of compactness, to check the following proposition

Proposition 2.8 Let $(X, d)$ be a metric space. Then $(X, d)$ is strongly complete if and only if $\mathrm{bd}\left({ }^{*} X\right)=\mathrm{ns}\left({ }^{*} X\right)$, where $\mathrm{bd}\left({ }^{*} X\right)$ denotes the set of bounded points in ${ }^{*} X$ (see Appendix C: Metric spaces). 
Proof of Theorem 2.5 Using Theorem 2.3, we only have to prove assertion (ii). Since the Kobayashi distance $d_{X}$ is an inner distance, we conclude that $X$ is in fact strongly complete for $d_{X}$. As a consequence, bounded points of * $X$ coincide with near standard points. Now let $z \in{ }^{*} \Delta$, such ${ }^{\circ}|z|<1$. By S-continuity, we get $f(\mu(z)) \subset \mu(f(z))$. Let $W$ be an S-neighborhood of $f(z)$ in ${ }^{*} \mathbb{C}^{n}$. Applying the permanence principle, there exists a standard positive real $r>0$ such that $f\left({ }^{*} \Delta_{r}\right) \subset W$. By Theorem 2.1, we conclude that the standard part ${ }^{\circ} f$ exists.

Let $X$ be a complex manifold and $H$ be a metric on $T X$; for simplicity, we will write $|v|$ instead of $H(v)$ for every $v \in T X$.

Proposition 2.9 The complex manifold $X$ is hyperbolic if and only if every internal holomorphic map $f:{ }^{*} \Delta \rightarrow{ }^{*} X$ with $f(0) \in \mathrm{ns}\left({ }^{*} X\right)$ verifies $\left|f^{\prime}(0)\right|$ is bounded.

Proof Suppose that $X$ is hyperbolic and let $f:{ }^{*} \Delta \rightarrow{ }^{*} X$ be an internal holomorphic map. Then $f$ is S-continuous in 0 . Hence for $W$ an S-neighborhood of $f(0)$, there exists a standard real $0<r<1$ such that $f\left({ }^{*} \Delta_{r}\right) \subset W$. By the Cauchy formula, we deduce that $\left|f^{\prime}(0)\right|$ is bounded. Conversely, if $X$ is not hyperbolic then by the Royden infinitesimal criterion of hyperbolicity, we conclude that there exist $x \in X$ and a sequence of holomorphic maps $f_{n}: \Delta \rightarrow X$ such that the sequence $\left(f_{n}(0)\right)$ converges to $x$ and $\left|f_{n}^{\prime}(0)\right| \rightarrow+\infty$. Clearly the sequence $\left(f_{n}\right)$ induces an internal holomorphic map $F:{ }^{*} \Delta \rightarrow{ }^{*} X$ verifying $F(0) \in \mathrm{ns}\left({ }^{*} X\right)$ and $\left|F^{\prime}(0)\right|$ is not bounded. -

Remark Proposition 2.9 is a nonstandard translation of the following characterization of hyperbolicity which asserts that a manifold $X$ is hyperbolic if and only if $X$ satisfies the Landau property (see for instance [9]), that is, for each $p \in X$ and each $W$ a relatively compact neighborhood of $p$, there exists $R>0$ such that

$$
\sup \left\{\left|f^{\prime}(0)\right|: f \in \operatorname{Hol}(\Delta, X) \text { with } f(0) \in W\right\} \leq R .
$$

Corollary 2.10 Let $X$ be a compact manifold. Then $X$ is hyperbolic if and only if every internal holomorphic map $f:{ }^{*} \Delta \longrightarrow^{*} X$ satisfies $\left|f^{\prime}(0)\right|$ is bounded.

We close this section by giving a nonstandard characterization of hyperbolicity in the compact case

Theorem 2.11 i) Let $Y$ be a relatively compact subspace of a complex space $Z$. Then $Y$ is hyperbolically embedded in $Z$ if and only if every internal holomorphic map $f:{ }^{*} \Delta \rightarrow{ }^{*} Y$ has a holomorphic standard part ${ }^{\circ} f: \Delta \rightarrow Z$. 
ii) Let $X$ be a compact complex space. Then $X$ is hyperbolic if and only if every internal holomorphic map $f:{ }^{*} \Delta \rightarrow{ }^{*} X$ has a holomorphic standard part ${ }^{\circ} f$ : $\Delta \rightarrow X$.

This is a nonstandard interpretation of the following standard facts:

i) Let $Y$ be a relatively compact subspace of a complex space $Z$. Then $Y$ is hyperbolically embedded in $Z$ if and only if $\operatorname{Hol}(\Delta, Y)$ is relatively compact in $\operatorname{Hol}(\Delta, Z)$.

ii) Let $X$ be a compact complex space. Then $X$ is hyperbolic if and only if $\operatorname{Hol}(\Delta, X)$ is compact.

\subsection{Bounded internal holomorphic functions}

Similarly, as for the algebra of bounded internal polynomials, we define ${ }^{b} \mathcal{O}\left(\mathbb{C}^{n}\right)$, the algebra (over ${ }^{b} \mathbb{C}$ ) of bounded entire $*$ - holomorphic functions on ${ }^{*} \mathbb{C}^{n}$, by

$$
{ }^{b} \mathcal{O}\left(\mathbb{C}^{n}\right)=\left\{f \in{ }^{*} \mathcal{O}\left(\mathbb{C}^{n}\right) \mid f\left({ }^{b} \mathbb{C}^{n}\right) \subset{ }^{b} \mathbb{C}\right\}
$$

Proposition 2.12 Let $f \in{ }^{b} \mathcal{O}\left(\mathbb{C}^{n}\right)$ be a bounded ${ }^{*}$-holomorphic function on ${ }^{*} \mathbb{C}^{n}$. Then ${ }^{\circ} f$, the standard of $f$ exists and is holomorphic on $\mathbb{C}^{n}$. We have for the zero sets, ${ }^{\circ} \mathrm{Z}(f) \subset \mathrm{Z}\left({ }^{\circ} f\right)$ and if ${ }^{\circ} f$ is not constant, then

$$
{ }^{\circ} \mathrm{Z}(f)=\mathrm{Z}\left({ }^{\circ} f\right) .
$$

Proof The first assertion is a direct consequence of Theorem 2.1. For the second, the inclusion follows from the S-continuity of $f$ and the S-openness of $f$ implies the equality.

Let now $X$ be a topological space. We denote by $\mathrm{ns}\left({ }^{*} X\right)$ the set of near-standard points of ${ }^{*} X$. The knowledge of $n s\left({ }^{*} X\right)$ is essential to define the standard part map.

We endow $\mathbb{C}\left[X_{1}, \ldots, X_{n}\right]$ and $\mathcal{O}\left(\mathbb{C}^{n}\right)$ with the compact-open topology, that is the topology of uniform convergence in each compact subset of $\mathbb{C}^{n}$.

For $g \in \mathcal{O}\left(\mathbb{C}^{n}\right)$, let $\mu(g)$ denote the halo of $g$ for the compact-open topology. 
Lemma 2.13 Let $g \in \mathcal{O}\left(\mathbb{C}^{n}\right)$. Then

$$
\mu(g)=\left\{f \in{ }^{*} \mathcal{O}\left(\mathbb{C}^{n}\right) \mid f(x) \approx{ }^{*} g(x) \text { for every } x \in{ }^{b} \mathbb{C}^{n}\right\} .
$$

In addition, we have ${ }^{b} \mathcal{O}\left(\mathbb{C}^{n}\right)=\mathrm{ns}\left({ }^{*} \mathcal{O}\left(\mathbb{C}^{n}\right)\right)$ and ${ }^{b} \mathbb{C}\left[X_{1}, \ldots, X_{n}\right]=\mathrm{ns}\left({ }^{*} \mathcal{O}\left(\mathbb{C}^{n}\right)\right) \cap$ $\mathbb{C}\left[X_{1}, \ldots, X_{n}\right]_{\text {int }}$.

Proof Let $\mathcal{C}\left(\mathbb{C}^{n}, \mathbb{C}\right)$ denote the set of continuous maps from $\mathbb{C}^{n}$ to $\mathbb{C}$ endowed with the compact-open topology. Let $g \in \mathcal{C}\left(\mathbb{C}^{n}, \mathbb{C}\right)$ and $\mu_{\mathcal{C}}(g)$ be the halo of $g$ in ${ }^{*} \mathcal{C}\left(\mathbb{C}^{n}, \mathbb{C}\right)$. Then we have that $\mu_{\mathcal{C}}(g)=\left\{f \in{ }^{*} \mathcal{C}\left(\mathbb{C}^{n}, \mathbb{C}\right) \mid f(x) \approx{ }^{*} g(x)\right.$ for every $\left.x \in{ }^{b} \mathbb{C}^{n}\right\}$, see Appendix C: Standard part of a map. Since $\mathcal{O}\left(\mathbb{C}^{n}\right) \subset \mathcal{C}\left(\mathbb{C}^{n}, \mathbb{C}\right)$ is equipped with the induced topology, then $\mu(g)=\mu_{\mathcal{C}}(g) \cap * \mathcal{O}\left(\mathbb{C}^{n}\right)$ for every $g \in \mathcal{O}\left(\mathbb{C}^{n}\right)$.

It is straightforward to verify that $\mathrm{ns}\left({ }^{*} \mathcal{O}\left(\mathbb{C}^{n}\right)\right) \subset{ }^{b} \mathcal{O}\left(\mathbb{C}^{n}\right)$, the converse inclusion is deduced from Proposition 2.12. -

Remark Put ${ }^{b} \mathcal{C}\left(\mathbb{C}^{n}, \mathbb{C}\right):=\left\{f \in{ }^{*} \mathcal{C}\left(\mathbb{C}^{n}, \mathbb{C}\right) \mid f\left({ }^{b} \mathbb{C}^{n}\right) \subset{ }^{b} \mathbb{C}\right\}$ and $\mathcal{S C}\left(\mathbb{C}^{n}, \mathbb{C}\right):=$ $\left\{f \in{ }^{*} \mathcal{C}\left(\mathbb{C}^{n}, \mathbb{C}\right)\right.$ S-continuous on $\left.{ }^{b} \mathbb{C}^{n}\right\}$. Then we have, by Appendix C: Standard part of a map,

$$
{ }^{b} \mathcal{C}\left(\mathbb{C}^{n}, \mathbb{C}\right) \cap \mathcal{S C}\left(\mathbb{C}^{n}, \mathbb{C}\right)=\operatorname{ns}\left({ }^{*} \mathcal{C}\left(\mathbb{C}^{n}, \mathbb{C}\right)\right)
$$

For $g \in \mathcal{O}\left(\mathbb{C}^{n}\right)$ let $\mu_{s}(g)$ denote the halo of $g$ in the topology of simple convergence. Then

$$
\mu_{s}(g)=\left\{f \in{ }^{*} \mathcal{O}\left(\mathbb{C}^{n}\right) \mid f(x) \approx g(x) \text { for every } x \in \mathbb{C}^{n}\right\}
$$

Now, we come to the central result of this section

Theorem 2.14 The standard part map defines a ring homomorphism

$$
\text { st: }{ }^{b} \mathcal{O}\left(\mathbb{C}^{n}\right) \longrightarrow \mathcal{O}\left(\mathbb{C}^{n}\right) .
$$

Its restriction to the subrings ${ }^{b} \mathbb{C}\left[X_{1}, \ldots, X_{n}\right]$ and ${ }^{b s} \mathbb{C}\left[X_{1}, \ldots, X_{n}\right]$ are surjective and we have the following commutative diagram

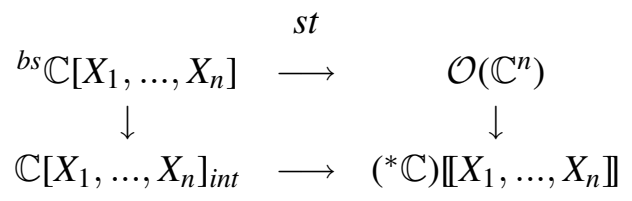

where the vertical arrows are the natural inclusions and the lower horizontal one is the ring homomorphism $\theta$ defined in Proposition 1.1. 
Proof The standard part map "st" is well-defined since we know that ${ }^{b} \mathcal{O}\left(\mathbb{C}^{n}\right)=$ $\mathrm{ns}\left({ }^{*} \mathcal{O}\left(\mathbb{C}^{n}\right)\right)$. It defines a ring homomorphism, because the standard part map is compatible with sums and products of complex numbers.

Now, we shall prove that the restriction of "st" to ${ }^{b s} \mathbb{C}\left[X_{1}, \ldots, X_{n}\right]$ is surjective. Let $f \in \mathcal{O}\left(\mathbb{C}^{n}\right)$, so

$$
f=\sum_{\nu \in \mathbb{N}^{n}} a_{\nu} X^{\nu} .
$$

The sequence $\left(a_{\nu}\right)_{\nu \in \mathbb{N}^{n}}$ extends to the internal sequence $\left(a_{\nu}\right)_{\nu \in \mathbb{N}^{n}}$. Let $N \in{ }^{*} \mathbb{N}_{\infty}$. Truncating $f$ at the order $N$, we get

$$
f_{N}=\sum_{|\nu| \leq N} a_{\nu} X^{\nu}
$$

and $f_{N}$ is an internal polynomial. Since the partial sums of $f$ converge on each compact of $\mathbb{C}^{n}$ to $f$, we have

$$
f_{N}(x) \approx{ }^{*} f(x) \text { for each } x \in{ }^{b} \mathbb{C}^{n}
$$

that is, $f_{N} \in \mu(f) \subset{ }^{b} \mathbb{C}\left[X_{1}, \ldots X_{n}\right]$. This shows $f_{N} \in{ }^{b s} \mathbb{C}\left[X_{1}, \ldots, X_{n}\right]$ and $\operatorname{st}\left(f_{N}\right)=f$.

The restriction of the map "st" to ${ }^{b} \mathbb{C}\left[X_{1}, \ldots, X_{n}\right]$ can be described explicitly. Let $f \in{ }^{b} \mathbb{C}\left[X_{1}, \ldots, X_{n}\right]$, of degree $d \in{ }^{*} \mathbb{N}$, and $f=\Sigma_{|\nu| \leq d} a_{\nu} X^{\nu}$. Then

$$
\operatorname{st}\left(\sum_{|\nu| \leq d} a_{\nu} X^{\nu}\right)=\sum_{\nu \in \mathbb{N}^{n}}{ }^{\circ}\left(a_{\nu}\right) X^{\nu}
$$

First, it is evident that the power series $g(x):=\Sigma_{\nu \in \mathbb{N}^{n}}{ }^{\circ}\left(a_{\nu}\right) x^{\nu}$ defines an entire holomorphic function on $\mathbb{C}^{n}$. In fact, let $\varepsilon>0$ be a standard positive real, there exists a finite integer $n_{0} \in \mathbb{N}$, such that $\left|a_{\nu}\right|^{\frac{1}{|\nu|}} \leq \varepsilon$ for each $\nu \in{ }^{*} \mathbb{N}^{n}$ such that $n_{0} \leq|\nu| \leq d$. Hence, for each standard $\nu \in \mathbb{N}^{n}$ such that $|\nu| \geq n_{0}$, we have ${ }^{\circ}\left|a_{\nu}\right|^{\frac{1}{|\nu|}} \leq \varepsilon$ which implies that $\lim \sup _{|\nu| \rightarrow+\infty}\left({ }^{\circ}\left|a_{\nu}\right|^{\frac{1}{|\nu|}}\right)=0$.

Trivially, the standard sequence of complex numbers $\left({ }^{\circ} a_{\nu}\right)_{\nu \in \mathbb{N}^{n}}$ extends to an internal sequence of complex numbers $\left(b_{n}\right)_{n \in * \mathbb{N}^{n}}$ such that for every standard $\nu$, we have $b_{\nu}=$ ${ }^{\circ} a_{\nu}$. By transfer, we get $\left|b_{\nu}\right|^{\frac{1}{|\nu|}} \leq \varepsilon$ for every $|\nu| \geq n_{0}, \nu \in{ }^{*} \mathbb{N}^{n}$. The uniform convergence of $g$ on each compact gives us

$$
{ }^{*} g(x) \approx \sum_{|\nu| \leq d} b_{\nu} x^{\nu} \text { for each } x \in{ }^{b} \mathbb{C}^{n} .
$$

We claim that $\Sigma_{|\nu| \leq d} a_{\nu} x^{\nu} \approx \Sigma_{|\nu| \leq d} b_{\nu} x^{\nu}$ for each $x \in{ }^{b} \mathbb{C}^{n}$. This is equivalent to $\Sigma_{|\nu| \leq d}\left(a_{\nu}-b_{\nu}\right) x^{\nu} \in{ }^{i} \mathbb{C}\left[X_{1}, \ldots, X_{n}\right]$. But this follows immediately from Proposition 1.4 which gives a characterization of infinitesimal bounded internal polynomials. 
Indeed, for each standard $\nu$, we have $a_{\nu} \approx b_{\nu}$ and $\left|a_{\nu}-b_{\nu}\right|^{\frac{1}{|\nu|}} \approx 0$ for infinite $\nu$, since $\left|a_{\nu}-b_{\nu}\right|^{\frac{1}{|\nu|}} \leq 2^{\frac{1}{|\nu|}} \varepsilon \leq 2 \varepsilon$ for every $\nu \in{ }^{*} \mathbb{N}^{n}, n_{0} \leq|\nu| \leq d$.

\subsection{The standard part functor in the affine case}

We intend to generalize 2.14 to bounded polynomial algebras, leading to Theorem 2.17 .

Let $n, m$ be finite positive integers and $i_{b}:{ }^{b} \mathbb{C}\left[X_{1}, \ldots, X_{n}\right] \hookrightarrow \mathbb{C}\left[X_{1}, \ldots, X_{n}\right]_{\text {int }}$ the inclusion of the algebra of bounded polynomials into the algebra of internal polynomials.

Proposition 2.15 Let $u$ be a ${ }^{*}$-homomorphism of ${ }^{*} \mathbb{C}$-algebras

$$
u: \mathbb{C}\left[X_{1}, \ldots, X_{n}\right]_{\text {int }} \longrightarrow \mathbb{C}\left[Y_{1}, \ldots, Y_{m}\right]_{\text {int }}
$$

such that $u\left(X_{i}\right)=g_{i} \in{ }^{b} \mathbb{C}\left[Y_{1}, \ldots, Y_{m}\right]$. Then we have

i) The homomorphism $u$ induces ${ }^{b} u:{ }^{b} \mathbb{C}\left[X_{1}, \ldots, X_{n}\right] \longrightarrow{ }^{b} \mathbb{C}\left[Y_{1}, \ldots, Y_{m}\right]$, a homomorphism of ${ }^{b} \mathbb{C}$-algebras such that $i_{b} \circ\left({ }^{b} u\right)=u \circ i_{b}$.

ii) The homomorphism ${ }^{b} u$ induces a map $\operatorname{st}\left({ }^{b} u\right)$ which is a homomorphism of Stein algebras between $\mathcal{O}\left(\mathbb{C}^{n}\right)$ and $\mathcal{O}\left(\mathbb{C}^{m}\right)$ such that $\mathrm{st} \circ\left({ }^{b} u\right)=\operatorname{st}\left({ }^{b} u\right) \circ$ st.

Proof Let $f$ be an internal polynomial, so $f=\Sigma_{|\nu| \leq d} a_{\nu} X_{1}^{\nu_{1}} \ldots X_{n}^{\nu_{n}}$. Then

$$
u(f)=\sum_{|\nu| \leq d} a_{\nu} g_{1}^{\nu_{1}} \ldots g_{n}^{\nu_{n}}=f\left(g_{1}, \ldots, g_{n}\right) .
$$

Assume now $f \in{ }^{b} \mathbb{C}\left[X_{1}, \ldots, X_{n}\right]$. It is clear that $u(f) \in{ }^{b} \mathbb{C}\left[Y_{1}, \ldots, Y_{m}\right]$ since if $x \in{ }^{b} \mathbb{C}^{n}$ then $\left(g_{1}(x), \ldots, g_{n}(x)\right) \in{ }^{b} \mathbb{C}^{n}$ and $f\left(g_{1}(x), \ldots, g_{n}(x)\right) \in{ }^{b} \mathbb{C}$.

As usual ${ }^{\circ} g_{1}, \ldots,{ }^{\circ} g_{n}$ stands for the standard parts of $g_{1}, \ldots, g_{n}$. Let $h \in \mathcal{O}\left(\mathbb{C}^{n}\right)$. We define $\left.\operatorname{st}^{b} u\right)$ by

$$
\operatorname{st}\left({ }^{b} u\right)(h):=h\left({ }^{\circ} g_{1}, \ldots,{ }^{\circ} g_{n}\right) .
$$

It is immediate that $\left.\operatorname{st}^{b} u\right)$ defines a homomorphism of Stein algebras between $\mathcal{O}\left(\mathbb{C}^{n}\right)$ and $\mathcal{O}\left(\mathbb{C}^{m}\right)$. Moreover, for every $N \in{ }^{*} \mathbb{N}_{\infty}$ we have $\operatorname{st}\left({ }^{b} u\right)(h)={ }^{\circ}\left(h_{N}\left(g_{1}, \ldots, g_{n}\right)\right)$, where $h_{N}$ denotes the Taylor expansion up to order $N$ of $h$. In other words, we get st $\left({ }^{b} u\right) \circ$ st $=$ st $\circ\left({ }^{b} u\right)$ on ${ }^{b} \mathbb{C}\left[X_{1}, \ldots, X_{n}\right]$, which was to show. - 
Now we shall define the category of "bounded" polynomial algebras (over $\mathbb{C}$ ). Objects are given by ${ }^{b} \mathbb{C}$-algebras of the form ${ }^{b} \mathbb{C}\left[X_{1}, \ldots, X_{n}\right] / I$ where $n \in \mathbb{N}$ and $I$ is an arbitrary ideal of ${ }^{b} \mathbb{C}\left[X_{1}, \ldots, X_{n}\right]$. Let $A={ }^{b} \mathbb{C}\left[X_{1}, \ldots, X_{n}\right] / I$ and $B={ }^{b} \mathbb{C}\left[Y_{1}, \ldots, Y_{m}\right] / J$ be two bounded polynomial algebras. A morphism between $A$ and $B$ is given by ${ }^{b} u:{ }^{b} \mathbb{C}\left[X_{1}, \ldots, X_{n}\right] \longrightarrow{ }^{b} \mathbb{C}\left[Y_{1}, \ldots, Y_{m}\right]$, a morphism of ${ }^{b} \mathbb{C}$-algebras induced by a n-uplets of bounded internal polynomials as in Proposition 2.15 which sends the ideal $I$ to the ideal $J$. In this way, we get a category of algebras which we call the category of bounded polynomial algebras. We note that there exist coproducts in this category.

We define the full subcategory of bounded polynomial algebras of finite type where objects are given by ${ }^{b} \mathbb{C}\left[X_{1}, \ldots, X_{n}\right] / I$ where $I$ is an ideal generated by finitely many bounded polynomials. Let $I_{\text {int }}:=i_{b}(I) . \mathbb{C}\left[X_{1}, \ldots, X_{n}\right]_{\text {int }}$ denote the ideal associated to $I$. Since the ideal $I$ is of finite type then $I_{\text {int }}$ is an internal ideal of $\mathbb{C}\left[X_{1}, \ldots, X_{n}\right]_{\text {int }}$. By our construction, we have the following proposition

Proposition 2.16 There is a natural covariant functor $\mathcal{F}$ from the category of bounded polynomial algebras of finite type (over $\mathbb{C}$ ) to the category of *-algebras of finite type over $\mathbb{C}$. This functor is compatible with coproducts.

Now we have developed the necessary tools to prove the main result of this section which is the construction of the standard part functor from the category of bounded polynomial algebras to the category of Stein algebras (over $\mathbb{C}$ ) of finite embedding dimension.

Theorem 2.17 There is an essentially surjective functor $\mathcal{S T}$, called the standard part functor, from the category of bounded polynomial algebras to the category of Stein algebras (over $\mathbb{C}$ ) of finite embedding dimension

$$
\mathcal{S T}: \text { (Bounded polynomial algebras }) \longrightarrow(\text { Stein algebras }) .
$$

Proof Let st: ${ }^{b} \mathbb{C}\left[X_{1}, \ldots X_{n}\right] \longrightarrow \mathcal{O}\left(\mathbb{C}^{n}\right)$ be the ring epimorphism defined in Theorem 2.14. Then ${ }^{\circ} I:=\operatorname{st}(\mathrm{I})$ is an ideal of $\mathcal{O}\left(\mathbb{C}^{n}\right)$ and its closure ${ }^{\bar{\sigma}} \boldsymbol{I}$ in $\mathcal{O}\left(\mathbb{C}^{n}\right)$ gives us a Stein algebra

$$
\mathcal{S} \mathcal{T}\left({ }^{b} \mathbb{C}\left[X_{1}, \ldots, X_{n}\right] / I\right):=\mathcal{O}\left(\mathbb{C}^{n}\right) / \overline{{ }^{\circ} I}
$$

Now, let $A={ }^{b} \mathbb{C}\left[X_{1}, \ldots, X_{n}\right] / I$ and $B={ }^{b} \mathbb{C}\left[Y_{1}, \ldots, Y_{m}\right] / J$ be two bounded polynomials algebras and ${ }^{b} u$ be the morphism between $A$ and $B$ which is given by n-uplets of bounded internal polynomials. By Proposition 2.15, st $\left({ }^{b} u\right)$ gives an homomorphism 
of Stein algebras between $\mathcal{O}\left(\mathbb{C}^{n}\right)$ and $\mathcal{O}\left(\mathbb{C}^{m}\right)$, satisfying $\operatorname{st}\left({ }^{b} u\right) \circ$ st $=$ st $\circ\left({ }^{b} u\right)$. Since st $\left({ }^{b} u\right)$ is continuous, we get

$$
\operatorname{st}\left({ }^{b} u\right)\left(\overline{{ }^{\circ}}\right) \subset \operatorname{st}\left(\overline{{ }^{\circ J}}\right) .
$$

Setting

$$
\mathcal{S T}\left({ }^{b} u\right): \mathcal{O}\left(\mathbb{C}^{n}\right) / \overline{{ }^{\circ} \boldsymbol{I}} \longrightarrow \mathcal{O}\left(\mathbb{C}^{m}\right) / \overline{{ }^{\circ} \mathrm{J}}
$$

which is given by $\left.\operatorname{st}^{b} u\right)$ modulo ${ }^{\overline{ }} \bar{I}$, we defined $\mathcal{S} \mathcal{T}$ on morphisms.

Now, let $C=\mathcal{O}\left(\mathbb{C}^{n}\right) / \mathfrak{a}$ be a Stein algebra. Then $\mathfrak{a}$ is a closed ideal of $\mathcal{O}\left(\mathbb{C}^{n}\right)$ generated by a family $\left(g_{i}\right)_{i \in I}$ of entire holomorphic functions over $\mathbb{C}^{n}$

$$
\mathfrak{a}=\sum_{i \in I} g_{i} \mathcal{O}\left(\mathbb{C}^{n}\right)
$$

For each $i \in I$, let $f_{i} \in{ }^{b} \mathbb{C}\left[X_{1}, \ldots, X_{n}\right]$ be a bounded internal polynomial such that ${ }^{\circ} f_{i}=g_{i}$. Define $\mathfrak{a}_{b}$ the ideal generated by the family $\left(f_{i}\right)_{i \in I}$ over ${ }^{b} \mathbb{C}\left[X_{1}, \ldots, X_{n}\right]$, that is

$$
\mathfrak{a}_{b}=\sum_{i \in I} f_{i}^{b} \mathbb{C}\left[X_{1}, \ldots, X_{n}\right] .
$$

Clearly we have ${ }^{\circ} \mathfrak{a}_{b}=\mathfrak{a}$ which implies that ${ }^{\circ} \mathfrak{a}_{b}=\mathfrak{a}$. Hence $\mathcal{S} \mathcal{T}\left({ }^{b} \mathbb{C}\left[X_{1}, \ldots, X_{n}\right] / \mathfrak{a}_{b}\right)=$ $\mathcal{O}\left(\mathbb{C}^{n}\right) / \mathfrak{a}$, which finishes the proof. -

If one wants to treat also the case of Stein algebras which are not necessarily of finite embedding dimension, one can proceed in the following way: Let $X$ be a Stein complex space. Then there exists an increasing sequence of natural numbers $n_{0}<n_{1}<\ldots$ and a projective system of holomorphic maps $\left(f_{k}: X \rightarrow \mathbb{C}^{n_{k}}\right)_{k \in \mathbb{N}}$ such that

a) the categorical image $Y_{k}$ of $f_{k}$ is a closed complex subspace of $\mathbb{C}^{n_{k}}$ and so we obtain a projective system $\left(Y_{k}\right)_{k}$ of Stein subspaces of $\left(\mathbb{C}^{n_{k}}\right)_{k}$,

b) for each compact $K \subset X$, there is a $k$ such that $f_{k}$ is an embedding of $K$; so we may consider in particular the map $X \rightarrow \lim _{\leftarrow} Y_{k}$ as a monomorphism and $\lim _{\rightarrow} \mathcal{O}\left(Y_{k}\right)$ as a dense subalgebra of $\mathcal{O}(X)$.

To these data, we can associate an algebra of internal bounded polynomials as follows: The sequence $\left(n_{0}, n_{1}, \ldots\right)$ defines an infinite natural number $N$ and so forth the ring of internal polynomials ${ }^{*} \mathbb{C}\left[X_{1}, \ldots, X_{N}\right]$. It contains $\lim _{\rightarrow k} b \mathbb{C}\left[X_{1}, \ldots, X_{n_{k}}\right]$ as a subring. The projective system $\left(Y_{k}\right)_{k}$ defines in an obvious (but non unique) way an ideal in this subring by fixing in addition an infinite natural number $N^{\prime}$, serving as a degree for replacing holomorphic functions by internal polynomials. So, we are able to "replace" the complex space $X$ by a certain type of algebra of internal polynomials. It is not 
difficult (but technical) to give a description in categorical terms of a standard part functor (which is essentially surjective), defined on this type of algebras and taking values in the category of Stein algebras. Since we do not need this construction in the sequel, we omit the details.

\subsection{Completions and enlargements}

The aim of this section is to compare the notions of completions and enlargements of topological rings. Let $(A, \mathfrak{I})$ be a topological ring and $\mathfrak{I}$ is an ideal of $\mathrm{A}$ which makes $A$ separated and complete for the $\mathfrak{I}$-adic topology, that is $\bigcap_{n>0} \mathfrak{I}^{n}=0$ and $A \cong \widehat{A}:=$ $\lim _{\leftarrow} A / \mathfrak{I}^{n+1}$. Let $\left({ }^{*} A,{ }^{*} \mathfrak{I}\right)$ be an enlargement of the couple $(A, \mathfrak{I})$. First, we prove that the ring ${ }^{*} A$ equipped with the ${ }^{*} \mathfrak{I}$-adic topology is in general not separated since $\mu(0):=\bigcap_{n>0} * \mathfrak{I}^{n}$, the halo of 0 , is not reduced to $\{0\}$, which occurs only if 0 is isolated in $A$, in other words if the ring $A$ is discrete. Let $\left.{ }^{*}\right) A:={ }^{*} A / \mu(0)$ denote the separated ring associated with ${ }^{*} A$. We show that ${ }^{(*)} A$ is complete for the ${ }^{*} \mathfrak{I}$-adic topology.

Before giving the proof, we shall prove first some results in a more general context and deduce from those the fact mentioned above.

Let $A$ be a ring and ${ }^{*} A:=\prod_{\mathcal{U}} A$ be an enlargement of $A$, where $\mathcal{U}$ is a non-principal ultrafilter on $\mathbb{N}$. Let $\mathfrak{a}$ be an internal ideal of ${ }^{*} A$.

Theorem 2.18 The canonical homomorphism of rings

$$
\theta:{ }^{*} A \longrightarrow \lim ^{*} A / \mathfrak{a}^{n+1}
$$

is surjective and its kernel is $\cap_{n>0} \mathfrak{a}^{n}$.

Proof The canonical homomorphism $\theta$ is defined by

$$
\theta(x)=\left(x_{k}:=x \bmod \mathfrak{a}^{k+1}\right)
$$

Let $\left(x_{k}\right)$ be a sequence of elements of ${ }^{*} A$, such that $x_{k+1}-x_{k} \in \mathfrak{a}^{k+1}$. Since the enlargement ${ }^{*} A$ is comprehensive, the sequence $\left(x_{k}\right)$ extends to an internal sequence $\left(x_{k}\right)_{k \in * \mathbb{N}}$, indexed by $* \mathbb{N}$. Let

$$
C=\left\{k \in{ }^{*} \mathbb{N} \mid x_{k+1}-x_{k} \in \mathfrak{a}^{k+1}\right\} .
$$

Then $C$ is an internal subset of ${ }^{*} \mathbb{N}$ which contains $\mathbb{N}$. Hence, by permanence, there exists $\omega_{0} \in{ }^{*} \mathbb{N}_{\infty}$ such that

$$
x_{k+1}-x_{k} \in \mathfrak{a}^{k+1} \text { for every } k \in \llbracket 1, \ldots, \omega_{0} \rrbracket .
$$


We put $y:=x_{\omega_{0}+1} \in{ }^{*} A$ and so we have

$$
y-x_{k}=\sum_{l=k}^{\omega_{0}}\left(x_{l+1}-x_{l}\right) \in \mathfrak{a}^{k+1} \text { for every } k \in \mathbb{N} .
$$

Then $\theta(y)=y \bmod \mathfrak{a}^{k+1}=x_{k} \bmod \mathfrak{a}^{k+1}$ which proves that $\theta$ is surjective. Clearly, $\operatorname{Ker}(\theta)=\cap_{n>0} \mathfrak{a}^{n}$.

Finally

$$
{ }^{*} A / \cap_{n>0} \mathfrak{a}^{n} \cong \lim ^{*} A / \mathfrak{a}^{n+1} .
$$

This shows that the separated space associated to ${ }^{*} A$ for the $\mathfrak{a}$-adic topology (i.e ${ }^{*} A / \cap$ $n>0 \mathfrak{a}^{n}$ ) is complete for this topology.

Remark By the permanence principle, we have $\cap_{k>0} \mathfrak{a}^{k}=\cup_{k \in \mathbb{N}_{\infty}} \mathfrak{a}^{k}$ and we conclude that $\cap_{k>0} \mathfrak{a}^{k} \neq 0$, if and only if, $\mathfrak{a}^{k+1} \neq 0$ for each $k \in \mathbb{N}$, which is the case if and only if $\mathfrak{a}^{k+1} \neq 0$ for some $k \in{ }^{*} \mathbb{N}_{\infty}$.

Using elementary proprieties of projective limits, we get

Corollary 2.19 Let $A$ be an $\mathfrak{I}$-adic ring. Then we have

i) $\lim _{\longleftarrow}{ }^{*} A /{ }^{*} \mathfrak{I}^{n+1} \cong * A / \mu(0)$,

ii) $\widehat{A} \hookrightarrow{ }^{*} A / \mu(0)$,

iii) $\widehat{A} \cong * A / \mu(0)$ if and only if $A / \mathfrak{I}^{n+1}$ is finite for every $n \in \mathbb{N}$.

Proof The first assertion is a direct consequence of Theorem 2.18. For the second one, by transfer, we have ${ }^{*}\left(A / \mathfrak{I}^{k}\right)={ }^{*} A /{ }^{*} \mathfrak{I}^{k}$. Consider the following sequence of projective systems (with surjective transition maps)

$$
0 \longrightarrow\left(A / \mathfrak{I}^{k}\right)_{k} \longrightarrow\left({ }^{*} A /^{*} \mathfrak{I}^{k}\right)_{k} \longrightarrow\left(\operatorname{Coker}\left(*_{k}\right)\right)_{k} \longrightarrow 0 .
$$

Taking the projective limit, we obtain the exact sequence

$$
0 \longrightarrow \lim _{\longleftarrow} A / \mathfrak{I}^{k+1} \longrightarrow \lim ^{*} A /^{*} \mathfrak{I}^{k+1} \longrightarrow \underset{\lim }{ } \operatorname{Coker}\left(*_{k+1}\right) \longrightarrow 0 .
$$

Therefore we get an injective homomorphism of rings $\widehat{A} \hookrightarrow{ }^{*} A / \mu(0)$ since $\widehat{A} \cong$ $\lim _{\longleftarrow}-A / \mathfrak{I}^{n+1}$ and $\lim _{\longleftarrow}{ }^{*} A /{ }^{*} \mathfrak{I}^{n+1} \cong{ }^{*} A / \mu(0)$. Furthermore, $\widehat{A}$ is (via this map) isomorphic to ${ }^{*} A / \mu(0)$ if and only if $\lim _{\longleftarrow} \operatorname{Coker}\left(*_{k+1}\right)=0$ if and only if the morphism $*_{k+1}: A / \mathfrak{I}^{k+1} \longrightarrow{ }^{*} A /{ }^{*} \mathfrak{I}^{k+1}$ is an isomorphism for each $k \in \mathbb{N}$; that is, if and only if $A / \mathfrak{I}^{k+1}$ is finite for every $k \in \mathbb{N}$. 
Example 2.20 Let $K$ be a field and $A=K\left[X_{1}, \ldots, X_{n}\right]$ be the ring of polynomials with coefficients in the field $K$ and $\mathfrak{M}$ be the maximal ideal generated by $\left(X_{1}, \ldots, X_{n}\right)$. Let ${ }^{*} A=K\left[X_{1}, \ldots, X_{n}\right]_{\text {int }}$ denote the ring of internal polynomials, so $\mu(0)=\cap_{k>0}\left(X_{1}, \ldots, X_{n}\right)^{k *} A$. The ring ${ }^{*} A / \mu(0)$ is isomorphic to a ring of power series in the standard sense $\Sigma_{k \geq 0} a_{\nu} X^{\nu}$ where $a_{\nu} \in{ }^{*} K$ and the sequence $\left(a_{\nu}\right)_{\nu \in \mathbb{N}^{n}}$ forms an initial segment of a hyperfinite sequence in ${ }^{*} K$. Hence, it is evident that $K \llbracket X_{1}, \ldots, X_{n} \rrbracket$, the ring of power series, is included in ${ }^{*} A / \mu(0)$. Furthermore, if the field $K$ is finite then $K \llbracket X_{1}, \ldots, X_{n} \rrbracket$ and ${ }^{*} A / \mu(0)$ are isomorphic.

\section{Affine *-schemes and *-bounded schemes}

\subsection{Affine *-schemes}

We now construct the category of affine nonstandard schemes and later that of "convergent" affine nonstandard schemes (which we call *-bounded schemes), more directly related to complex spaces. Our approach is self-contained and independent of the paper [2]. There, the authors defined the functor ${ }^{*}$ Spec from the category ${ }^{*}($ Rings $)$ the category of internal rings to ${ }^{*}$ (Aff. Sch), the category of internal affine schemes, and *-affine schemes as the essential image of ${ }^{*}$ Spec.

In this section, we equip in particular these objects by a topology and a canonical sheaf structure. As a consequence, ${ }^{*}$-affine schemes form a subcategory of the category of locally ringed spaces.

Let $I$ be an infinite set and let $\mathcal{U}$ be a non-principal ultrafilter on $I$.

\section{The internal spectrum of an internal ring}

Since the index set $I$ will be fixed in the sequel, we will write ${ }^{*} X$ instead of ${ }^{*} X_{I}$ for the ultraproduct of a system of sets $\left(X_{i}\right)_{i \in I}$.

Let ${ }^{*} R=\prod_{\mathcal{U}} R_{i}$ be a ${ }^{*}$-commutative ${ }^{*}$-ring. Then ${ }^{*} R$ is a commutative ring and $\operatorname{Spec}\left({ }^{*} R\right)$ denotes the (usual) spectrum of ${ }^{*} R$. We define

$$
\operatorname{Specint}\left({ }^{*} R\right)=\left\{\mathfrak{J} \in \operatorname{Spec}\left({ }^{*} R\right) \mid \mathfrak{J} \text { is an internal ideal of }{ }^{*} R\right\} .
$$

By transfer, we easily prove

i) ${ }^{*} R$ is an integral domain if and only if $\left\{i \in I \mid R_{i}\right.$ is an integral domain $\} \in \mathcal{U}$,

ii) ${ }^{*} R$ is a field if and only if $\left\{i \in I \mid R_{i}\right.$ is a field $\} \in \mathcal{U}$. 
As a consequence, we get

Proposition 3.1 With the above notations, we have natural bijections

i) $\quad \operatorname{Specint}\left({ }^{*} R\right) \cong \prod_{\mathcal{U}} \operatorname{Spec}\left(R_{i}\right)$,

ii) $\operatorname{Specmaxint}\left({ }^{*} R\right) \cong \prod_{\mathcal{U}} \operatorname{Specmax}\left(R_{i}\right)$.

In particular, let $k$ be an algebraic closed field, then by the Hilbert Nullstellensatz, we have $\operatorname{Specmaxint}\left(k\left[T_{1}, \ldots, T_{n}\right]_{\text {int }}\right) \simeq{ }^{*} k^{n}$.

Let $j: \operatorname{Specint}\left({ }^{*} R\right) \longrightarrow \operatorname{Spec}\left({ }^{*} R\right)$ denote the inclusion map.

We endow Specint $\left({ }^{*} R\right)$ with the induced Zariski topology, defined on $\operatorname{Spec}\left({ }^{*} R\right)$. Hence closed subsets of $\operatorname{Specint}\left({ }^{*} R\right)$ are given by

$$
\mathcal{V}(\mathfrak{a})=\left\{\mathfrak{J} \in \operatorname{Specint}\left({ }^{*} R\right) \mid \mathfrak{J} \supset \mathfrak{a}\right\}
$$

where $\mathfrak{a}$ is an ideal of ${ }^{*} R$ which may be external.

Let $\mathfrak{J}$ be an internal ideal of ${ }^{*} R$. We set

$$
\sqrt[i n t]{\mathfrak{J}}=\left\{f \in{ }^{*} R \mid \exists n \in{ }^{*} \mathbb{N}, f^{n} \in \mathfrak{J}\right\} .
$$

Then $\sqrt[i n t]{\mathfrak{J}}$ is an ideal of ${ }^{*} R$, containing $\mathfrak{J}$.

If $\mathfrak{J}$ is an internal ideal of ${ }^{*} R$, so $\mathfrak{J}=\prod_{\mathcal{U}} \mathfrak{J}_{i}$, then $\sqrt[i n t]{\mathfrak{J}}$ is internal too and given by $\sqrt[i n t]{\mathfrak{J}}=\prod_{\mathcal{U}} \sqrt{\mathfrak{J}_{i}}$. Again by transfer, we conclude that $\sqrt[i n t]{\mathfrak{J}}$ is the intersection of all internal prime ideals containing $\mathfrak{J}$; that is,

$$
\sqrt[i n t]{\mathfrak{J}}=\bigcap_{\mathfrak{p} \in \operatorname{Specint}\left({ }^{*} R\right), \mathfrak{p} \supset \mathfrak{J}} \mathfrak{p}
$$

We have

\section{Proposition 3.2}

i) Let $\mathfrak{a}=\Pi_{\mathcal{U}} \mathfrak{a}_{i}$ be an internal ideal of ${ }^{*} R$. Then $\mathcal{V}(\mathfrak{a})$ is an internal subset of $\operatorname{Specint}\left({ }^{*} R\right)$ and $\mathcal{V}(\mathfrak{a})=\Pi_{\mathcal{U}} V\left(\mathfrak{a}_{i}\right)$ where $V\left(\mathfrak{a}_{i}\right)=\left\{\mathfrak{J} \in \operatorname{Spec}\left(R_{i}\right) \mid \mathfrak{J} \supset \mathfrak{a}_{i}\right\}$.

ii) Let $\mathfrak{a}, \mathfrak{b}$ be two internal ideals in ${ }^{*} R$. Then $\sqrt[i n t]{\mathfrak{b}} \subset \sqrt[i n t]{\mathfrak{a}}$ if and only if $\mathcal{V}(\mathfrak{a}) \subset \mathcal{V}(\mathfrak{b})$. 
Proof i) Let $\mathfrak{J}=\Pi_{\mathcal{U}} \mathfrak{J}_{i}$ be an internal ideal of ${ }^{*} R$. By transfer, we have $\mathfrak{J} \supset \mathfrak{a}$ if and only if $\left\{i \in I \mid \mathfrak{J}_{i} \supset \mathfrak{a}_{i}\right\} \in \mathcal{U}$ which is equivalent to $\left\{i \in I \mid \mathfrak{J}_{i} \in V\left(\mathfrak{a}_{i}\right)\right\} \in \mathcal{U}$.

ii) Let $\mathfrak{p}$ be an internal ideal containing $\mathfrak{a}$. Then $\mathfrak{p}$ contains $\sqrt[i n t]{\mathfrak{a}}$, hence $\mathfrak{p} \supset \sqrt[i n t]{\mathfrak{b}} \supset \mathfrak{b}$. The converse is, via transfer, an easy consequence, since $\mathcal{V}(\mathfrak{a}) \subset \mathcal{V}(\mathfrak{b})$ if an only if $\left\{i \in I \mid V\left(\mathfrak{a}_{i}\right) \subset V\left(\mathfrak{b}_{i}\right)\right\} \in \mathcal{U}$ if and only if $\left\{i \in I \mid \sqrt{\mathfrak{b}_{i}} \subset \sqrt{\mathfrak{a}_{i}}\right\} \in \mathcal{U}$ which means $\sqrt[i n t]{\mathfrak{b}} \subset \sqrt[i n t]{\mathfrak{a}}$.

We fix $f=\Pi_{\mathcal{U}} f_{i} \in{ }^{*} R$, and put

$$
\mathfrak{D}(f)=\left\{\mathfrak{p} \in \operatorname{Specint}\left({ }^{*} R\right) \mid f \notin \mathfrak{p}\right\} .
$$

Then $\mathfrak{D}(f)=\Pi_{\mathcal{U}} D\left(f_{i}\right)$, where $D\left(f_{i}\right)=\left\{\mathfrak{p} \in \operatorname{Spec}\left(R_{i}\right) \mid f_{i} \notin \mathfrak{p}\right\}$.

The sets $\left\{\mathfrak{D}(f) \mid f \in{ }^{*} R\right\}$ form an internal open base for the induced Zariski topology, induced by $\operatorname{Spec}\left({ }^{*} R\right)$. For any ideal $\mathfrak{I}$ in ${ }^{*} R$, we have $\operatorname{Specint}\left({ }^{*} R\right) \backslash \mathcal{V}(\mathfrak{I})=\cup_{f \in \mathfrak{I}} \mathfrak{D}(f)$.

Since $\mathfrak{D}(f)$ are internal subsets of $\operatorname{Specint}\left({ }^{*} R\right)$, we have

Proposition 3.3 Let $f=\Pi_{\mathcal{U}} f_{i}$, and $g=\Pi_{\mathcal{U}} g_{i}$ be two elements of ${ }^{*} R$. Then

i) $\mathfrak{D}(f)=\emptyset$ if and only if there exists $n \in{ }^{*} \mathbb{N}$ such that $f^{n}=0$,

ii) $\mathfrak{D}(f) \cap \mathfrak{D}(g)=\mathfrak{D}(f g)$ and for each $n \in{ }^{*} \mathbb{N}$ positive, we have $\mathfrak{D}\left(f^{n}\right)=\mathfrak{D}(f)$,

iii) $\mathfrak{D}(f) \supset \mathfrak{D}(g)$ if and only if $g \in \sqrt[i n t]{(f)}$, which is equivalent to $\left\{i \in I \mid D\left(f_{i}\right) \supset D\left(g_{i}\right)\right\} \in \mathcal{U}$.

Moreover, we have

Proposition 3.4 Let ${ }^{*} S=\Pi_{\mathcal{U}} S_{i}$ be an internal subset of ${ }^{*} R$. Then ${ }^{*} S$ is a multiplicative subset of ${ }^{*} R$ if and only if $\left\{i \in I \mid S_{i}\right.$ is multiplicative in $\left.R_{i}\right\} \in \mathcal{U}$ and ${ }^{*} S^{-1}\left({ }^{*} R\right)=\Pi_{\mathcal{U}} S_{i}^{-1} R_{i}$.

We consider two examples of internal multiplicative subsets of ${ }^{*} R$

i) Let $\mathfrak{p}=\Pi_{\mathcal{U}} \mathfrak{p}_{i} \in \operatorname{Specint}\left({ }^{*} R\right)$ be an internal prime ideal of ${ }^{*} R$. Then ${ }^{*} R \backslash \mathfrak{p}=$ $\Pi_{\mathcal{U}} R_{i} \backslash \mathfrak{p}_{i}$ is an internal multiplicative subset of ${ }^{*} R$ and $\left({ }^{*} R\right)_{\mathfrak{p}}={ }^{*} S^{-1}\left({ }^{*} R\right)=$ $\Pi_{\mathcal{U}}\left(R_{i}\right)_{\mathfrak{p}_{i}}$.

ii) Let $f=\Pi_{\mathcal{U}} f_{i} \in{ }^{*} R$ and ${ }^{*} S=\left\{f^{N}, N \in{ }^{*} \mathbb{N}\right\}$. Clearly ${ }^{*} S$ is an internal multiplicative subset of ${ }^{*} R$ and ${ }^{*} S=\Pi_{\mathcal{U}} S_{i}$, where $S_{i}=\left\{f_{i}^{n}, n \in \mathbb{N}\right\}$. 
We denote by

$$
{ }^{*} R_{[f]}={ }^{*} S^{-1}\left({ }^{*} R\right)=\Pi_{\mathcal{U}}\left(R_{i}\right)_{f_{i}}
$$

the localization of ${ }^{*} R$ with respect to ${ }^{*} S$ which is an internal ring. Its internal prime spectrum is given by

Proposition 3.5 Let $f \in{ }^{*} R$. Then $\operatorname{Specint}\left({ }^{*} R_{[f]}\right)=\mathfrak{D}(f)=\operatorname{Specint}\left({ }^{*} R\right) \backslash \mathcal{V}(f)$.

Proof We identify naturally

$\operatorname{Specint}\left({ }^{*} R_{[f]}\right)=\operatorname{Specint}\left(\Pi_{\mathcal{U}}\left(R_{i}\right) f_{i}\right)=\Pi_{\mathcal{U}} \operatorname{Spec}\left(\left(R_{i}\right)_{f_{i}}\right)=\Pi_{\mathcal{U}} D\left(f_{i}\right)=\mathfrak{D}(f)$.

Let ${ }^{*} R_{f}$ denote the localization of the ring ${ }^{*} R$ with respect to the multiplicative family $\left\{1, f, f^{2}, \ldots, f^{n}, \ldots, n \in \mathbb{N}\right\}$. Then there is a natural morphism of rings

$$
{ }^{*} R_{f} \longrightarrow{ }^{*} R_{[f]}
$$

induced by the identity.

\section{Structure sheaf of an internal prime spectrum}

Following the classical procedure, we define a sheaf of commutative rings over ${ }^{*} X:=$ Specint $\left({ }^{*} R\right)$, the internal prime spectrum, equipped with the internal topology of Zariski. We first define sections and restriction maps on the sets $\mathfrak{D}(f), f \in{ }^{*} R$, which form a base for the Zariski topology, induced by that of $\operatorname{Spec}\left({ }^{*} R\right)$. We set

$$
\mathcal{A}^{* X}(\mathfrak{D}(f)):={ }^{*} R_{[f]} .
$$

Clearly, this defines a presheaf on ${ }^{*} X$, where restriction maps on elements of the base are given as follows: Let $\mathfrak{D}(f) \supset \mathfrak{D}(g)$ which is equivalent to $J:=\left\{i \in I \mid D\left(f_{i}\right) \supset\right.$ $\left.D\left(g_{i}\right)\right\} \in \mathcal{U}$. There is an internal homomorphism of internal rings

$$
{ }^{*} \rho_{\mathfrak{D}(g), \mathfrak{D}(f)}:{ }^{*} R_{[f]} \longrightarrow{ }^{*} R_{[g]}
$$

induced by the restriction maps $\rho_{D\left(g_{i}\right), D\left(f_{i}\right)}:\left(R_{i}\right)_{f_{i}} \longrightarrow\left(R_{i}\right)_{g_{i}}$ for every $i \in J$. Trivially, we have ${ }^{*} \rho_{\mathfrak{D}(f), \mathfrak{D}(f)}=i d$ and ${ }^{*} \rho_{\mathfrak{D}(h), \mathfrak{D}(g)} \circ^{*} \rho_{\mathfrak{D}(g), \mathfrak{D}(f)}={ }^{*} \rho_{\mathfrak{D}(h), \mathfrak{D}(f)}$, for $\mathfrak{D}(f) \supset \mathfrak{D}(g) \supset$ $\mathfrak{D}(h)$. Consider the collection of internal open sets $\mathfrak{D}(f)$, containing $\mathfrak{p} \in \operatorname{Specint}\left({ }^{*} R\right)$. We put $\mathcal{W}_{\mathfrak{p}}=\{\mathfrak{D}(f), \mathfrak{p} \in \mathfrak{D}(f)\}$. It is a directed set.

Let ${ }^{*} \rho_{\mathfrak{p}}^{f}:{ }^{*} R_{[f]} \longrightarrow{ }^{*} R_{\mathfrak{p}}$ be the canonical internal homomorphism for each $\mathfrak{p} \in \mathfrak{D}(f)$. The following proposition is immediate 
Proposition 3.6 For an internal prime ideal $\mathfrak{p}$ of an internal ring ${ }^{*} R$, there is a natural isomorphism of rings

$$
\lim _{\rightarrow}^{*} R_{[f]} \rightarrow{ }^{*} R_{\mathfrak{p}}
$$

where the limit is taken over all $f$ such that $\mathfrak{p} \in \mathfrak{D}(f)$.

Proof We can use transfer to prove this by constructing an isomorphism between $\lim _{\rightarrow}{ }^{*} R_{[f]}$ and $\Pi_{\mathcal{U}} \lim _{\rightarrow}\left(R_{i}\right)_{f_{i}}$. The last one is internally isomorphic to $\Pi_{\mathcal{U}}\left(R_{i}\right)_{\mathfrak{p}_{i}}=$ ${ }^{*} R_{\mathfrak{p}}$, where $\mathfrak{p}=\Pi_{\mathcal{U}} \mathfrak{p}_{i} \in \mathfrak{D}(f)$, so we are done.

We show now, using transfer, that our sheaf is already separated:

Lemma 3.7 Let $f \in{ }^{*} R$ and $\mathfrak{D}(f)=\cup_{\alpha \in A} \mathfrak{D}\left(f_{\alpha}\right)$. Suppose that for $a \in{ }^{*} R_{[f]}$

$$
{ }^{*} \rho_{\mathfrak{D}\left(f_{\alpha}\right), \mathfrak{D}(f)}(a)=0 \quad \text { for each } \alpha \in A .
$$

Then $a=0$.

Proof The element $a \in{ }^{*} R_{[f]}$ can be expressed in the form $a=g / f^{m}$, where $m$ is a hyperinteger. If $\mathfrak{J}=\left\{h \in{ }^{*} R \mid h g=0\right\}$, then $\mathfrak{J}$ is an internal ideal of ${ }^{*} R$ and $a=0$ in ${ }^{*} R_{[f]}$ if and only if $f \in \sqrt[i n t]{\mathfrak{J}}=\cap_{\mathfrak{J} \subset \mathfrak{p} \in \operatorname{Specint}\left({ }^{*} R\right)} \mathfrak{p}$.

Suppose that $a \neq 0$ in ${ }^{*} R_{[f]}$. Then there exists an internal prime ideal $\mathfrak{p} \supset \mathfrak{J}$ with $f \notin \mathfrak{p}$; that is, $\mathfrak{p} \in \mathfrak{D}(f)$. We take $\alpha \in A$ such that $\mathfrak{p} \in \mathfrak{D}\left(f_{\alpha}\right)$ and ${ }^{*} \rho_{\mathfrak{p}}^{f_{\alpha}} \circ{ }^{*} \rho_{\mathfrak{D}\left(f_{\alpha}\right), \mathfrak{D}(f)}=$ ${ }^{*} \rho_{\mathfrak{p}}^{f}$. By assumption, the image of $g=f^{m} a$ in ${ }^{*} R_{\mathfrak{p}}$ is zero, which means, there exists $b \in R \backslash \mathfrak{p}$ such that $b g=0$. Hence $b \in \mathfrak{J}$, which contradicts $b \in R \backslash \mathfrak{p}$, since $\mathfrak{p} \supset \mathfrak{J}$.

Definition 3.8 The sheaf of rings associated to the presheaf $\mathcal{A}^{*}$, given by

$$
\mathcal{A}^{* X}(\mathfrak{D}(f)):={ }^{*} R_{[f]}
$$

on the basis $\left\{\mathfrak{D}(f) \mid f \in{ }^{*} X\right\}$ of ${ }^{*} X$, will be called the structure sheaf on the internal spectrum ${ }^{*} X=\operatorname{Specint}\left({ }^{*} R\right)$, and denoted by $\mathcal{O}^{*} X$.

Let $\theta_{f}:{ }^{*} R_{[f]} \longrightarrow \Gamma\left(\mathfrak{D}(f), \mathcal{O}^{*} X\right)$ be the canonical map. It is given as follows: For $\mathfrak{p} \in \mathfrak{D}(f)$ and $\xi \in{ }^{*} R_{[f]}$, we have $\left(\theta_{f}(\xi)\right)_{\mathfrak{p}}={ }^{*} \rho_{\mathfrak{p}}^{f}(\xi)$. So by (3.7), we get

Corollary 3.9 For every $f \in{ }^{*} R$, the homomorphism $\theta_{f}:{ }^{*} R_{[f]} \longrightarrow \Gamma\left(\mathfrak{D}(f), \mathcal{O}^{*} X\right)$ is injective. 
Let ${ }^{*} R_{f} \longrightarrow \Gamma\left(\mathfrak{D}(f), \mathcal{O}_{* X}\right)$ be the homomorphism of rings, given by composing ${ }^{*} R_{f} \longrightarrow{ }^{*} R_{[f]}$ and $\theta_{f}$. These homomorphisms induce a morphism of sheaves

$$
\mathcal{O}_{\operatorname{Spec}\left({ }^{*} R\right)} \longrightarrow j_{*} \mathcal{O}_{\operatorname{Specint}\left({ }^{*} R\right)}
$$

and so we obtain $j$ as a morphism of ringed spaces. The following proposition allows us to interpret $j$ as an open immersion

Proposition 3.10 The sheaves $\mathcal{O}^{*} X$ and $j^{-1}\left(\mathcal{O}_{\operatorname{Spec}\left({ }^{*} R\right)}\right)$ are naturally isomorphic.

Proof Applying the functor $j^{-1}$ to $\mathcal{O}_{\operatorname{Spec}\left({ }^{*} R\right)} \longrightarrow j_{*} \mathcal{O}_{*} X$, we get a morphism of sheaves $j^{-1} \mathcal{O}_{\operatorname{Spec}\left({ }^{*} R\right)} \longrightarrow \mathcal{O}^{*} X$ on $\operatorname{Specint}\left({ }^{*} R\right)$. This morphism is an isomorphism since it gives the identity on stalks at every internal prime ideal of ${ }^{*} R$.

The interplay between the diverse spectra can be summarized as follows :

Let $R$ be a ring and we denote by ${ }^{*} R=\Pi_{\mathcal{U}} R$ its ultrapower. The morphism of rings * $R \longrightarrow{ }^{*} R$ induces a continuous map

$$
\operatorname{Spec}(*): \operatorname{Spec}\left({ }^{*} R\right) \longrightarrow \operatorname{Spec}(R)
$$

where $\operatorname{Spec}\left({ }^{*} R\right)$ and $\operatorname{Spec}(R)$ are equipped with the Zariski topology. We denote by

$$
\operatorname{Specint}(*): \operatorname{Specint}\left({ }^{*} R\right) \longrightarrow \operatorname{Spec}(R)
$$

the restriction of $\operatorname{Spec}(*)$ to $\operatorname{Specint}\left({ }^{*} R\right)$ which carries the induced Zariski topology of $\operatorname{Spec}\left({ }^{*} R\right)$. Clearly, $\operatorname{Specint}(*)$ is continuous. We should mention that the induced topology on $\operatorname{Specint}\left({ }^{*} R\right)={ }^{*} \operatorname{Spec}(R)$ coincides with the so-called Q-topology since they have the same basis, given by the *-open subsets $\mathfrak{D}(f)=\Pi_{\mathcal{U}} D\left(f_{i}\right)$. The enlargement construction gives an injective map

$$
\text { *: } \operatorname{Spec}(R) \longrightarrow{ }^{*} \operatorname{Spec}(R)
$$

$\mathfrak{p} \mapsto * \mathfrak{p}$. This map is not continuous since the restriction of the Q-topology does not coincide with the initial one. So, it is more natural to work with $\operatorname{Specint}(*)$ on the level of topological spaces.

Now, we will define the category of affine ${ }^{*}$-schemes. An object is given by a locally ringed space $\left({ }^{*} X, \mathcal{O}^{*} X\right)$ with the underlying topological space ${ }^{*} X:=\operatorname{Specint}\left({ }^{*} \mathrm{R}\right)$ where ${ }^{*} R$ is an internal ring and $\mathcal{O}^{*} X=j_{*} \mathcal{O}_{\operatorname{Spec}\left({ }^{*} R\right)}$ (the mapping $j: \operatorname{Specint}\left({ }^{*} R\right) \rightarrow$ $\operatorname{Spec}\left({ }^{*} R\right)$ denotes the inclusion). Let $\left({ }^{*} Y, \mathcal{O}^{*} Y\right)$ be another object in the category of ${ }^{*}$-affine schemes defined by an internal ring ${ }^{*} S$, that is, ${ }^{*} Y=\operatorname{Specint}\left({ }^{*} S\right)$. A morphism between $\left({ }^{*} X, \mathcal{O}^{*} X\right)$ and $\left({ }^{*} Y, \mathcal{O}^{*} Y\right)$ is represented by an internal morphism of 
rings $\varphi:{ }^{*} S \rightarrow{ }^{*} R$ which induces a morphism of locally ringed space as follows: the restriction of $\operatorname{Spec}(\varphi)$ on $\operatorname{Specint}\left({ }^{*} R\right)$ gives a continuous mapping

$$
\operatorname{Specint}(\varphi): \operatorname{Specint}\left({ }^{*} R\right) \rightarrow \operatorname{Specint}\left({ }^{*} S\right)
$$

and, by localization, $\varphi$ gives a morphism of sheaves

$$
\mathcal{O}_{*_{Y}} \rightarrow \operatorname{Specint}(\varphi)_{*}\left(\mathcal{O}_{*_{X}}\right)
$$

\subsection{Affine*-bounded schemes}

There is a natural functor between the category of affine schemes of finite type over $\mathbb{C}$ and the category of Stein spaces. This functor associates for each algebra of finite type $A=\mathbb{C}\left[X_{1}, \ldots, X_{n}\right] /\left(f_{1}, \ldots, f_{q}\right)$ the Stein algebra $\mathcal{O}\left(\mathbb{C}^{n}\right) / \sum_{i=1}^{q} f_{i} \mathcal{O}\left(\mathbb{C}^{n}\right)$, where each polynomial $f_{i}$ is considered as an entire holomorphic function over $\mathbb{C}^{n}$.

In this section, we will construct the category of affine bounded *-schemes as an "intermediate" category between the category of affine schemes of finite type over $\mathbb{C}$ and the category of Stein spaces. This category will be a subcategory of locally ringed spaces.

Before giving this construction, we recall some facts and notations on the topology of ${ }^{b} \mathbb{C}^{n}$, the space of bounded points of ${ }^{*} \mathbb{C}^{n}$. The space ${ }^{b} \mathbb{C}^{n}$ is an S-closed and S-open subspace of ${ }^{*} \mathbb{C}^{n}$. A basis for its topology is given by the S-balls $S(p, r)=\{q \in$ $\left.{ }^{b} \mathbb{C}^{n}\left|{ }^{\circ}\right| q-p \mid<r\right\}$, where $p \in{ }^{b} \mathbb{C}^{n}$ and $r$ is a positive real standard number. As a consequence, the standard part mapping

$$
\text { st : }{ }^{b} \mathbb{C}^{n} \rightarrow \mathbb{C}^{n}
$$

is continuous and open. Furthermore, we note that the inclusion mapping

$$
i: \mathbb{C}^{n} \rightarrow{ }^{b} \mathbb{C}^{n}
$$

is continuous. If fact, the S-topology coincides with the initial topology which makes the standard mapping st : ${ }^{b} \mathbb{C}^{n} \rightarrow \mathbb{C}^{n}$ continuous. Finally, we note that if $U$ is S-open, then ${ }^{\circ} U$ is an open subset of $\mathbb{C}^{n}$ and ${ }^{\circ} U=U \cap \mathbb{C}^{n}$.

Let $\mathfrak{a}$ be an ideal of ${ }^{b} \mathbb{C}\left[X_{1}, \ldots, X_{n}\right]$. Define the variety of $\mathfrak{a}$ by

$$
V(\mathfrak{a})=\left\{x \in{ }^{b} \mathbb{C}^{n} \mid f(x) \approx 0 \text { for each } f \in \mathfrak{a}\right\} .
$$

Clearly, $V(\mathfrak{a})$ is $\mathrm{S}$-closed, since each bounded polynomial is S-continuous. Again, by S-continuity, we have $V(\mathfrak{a})=\left\{x \in{ }^{b} \mathbb{C}^{n} \mid f(y) \approx 0\right.$ for each $\left.f \in \mathfrak{a}, y \in \mu(x)\right\}$. 
Let $\mathfrak{b}$ be a subset of $\mathcal{O}\left(\mathbb{C}^{n}\right)$. Define the zero set of $\mathfrak{b}$ by

$$
Z(\mathfrak{b})=\left\{x \in \mathbb{C}^{n} \mid f(x)=0 \text { for each } f \in \mathfrak{b}\right\} .
$$

For each ideal $\mathfrak{a}$ of ${ }^{b} \mathbb{C}\left[X_{1}, \ldots, X_{n}\right]$, we set ${ }^{\circ} \mathfrak{a}=\operatorname{st}(\mathfrak{a})$ which is an ideal of $\mathcal{O}\left(\mathbb{C}^{n}\right)$, its standard part.

We have the following rules

Proposition 3.11 Let $\left(\mathfrak{a}_{i}\right)_{i \in I}$ be a family of ideals and $\mathfrak{a}, \mathfrak{b}$ two ideals all in ${ }^{b} \mathbb{C}[X]$. Moreover, we fix $f \in{ }^{b} \mathbb{C}[X]$. Then

i) $\cap_{i \in I} V\left(\mathfrak{a}_{i}\right)=V\left(\sum_{i \in I} \mathfrak{a}_{i}\right)$,

ii) $V(\mathfrak{a}) \cup V(\mathfrak{b})=V(\mathfrak{a} \cap \mathfrak{b})=V(\mathfrak{a} \mathfrak{b})$,

iii) ${ }^{\circ} V(\mathfrak{a})=Z\left({ }^{\circ} \mathfrak{a}\right)$ and $i^{-1}(V(\mathfrak{a}))=Z\left({ }^{\circ} \mathfrak{a}\right)$,

iv) $V\left(\mathfrak{a}+{ }^{i} \mathbb{C}[X]\right)=V(\mathfrak{a})$,

v) $V(\mathfrak{a}) \subset V(\mathfrak{b})$ if and only if $Z\left({ }^{\circ} \mathfrak{a}\right) \subset Z\left({ }^{\circ} \mathfrak{b}\right)$,

vi) $V(f)=\emptyset$ if and only if ${ }^{\circ} f(x) \neq 0$ for every $x \in \mathbb{C}^{n}$,

vii) $V(f)={ }^{b} \mathbb{C}^{n}$ if and only if $f \in{ }^{i} \mathbb{C}[X]$.

Proof The assertions (i), (ii), (iv), (vi) and (vii) are straightforward. For the assertion (iii), the inclusion $Z\left({ }^{\circ} \mathfrak{a}\right) \subset{ }^{\circ} V(\mathfrak{a})$ is obvious. Conversely, since each element $f \in \mathfrak{a}$ is S-continuous, we have ${ }^{\circ} V(\mathfrak{a}) \subset Z\left({ }^{\circ} \mathfrak{a}\right)$. The same argument about S-continuity together with (iii) prove (v).

Remark The sets $V(\mathfrak{a})$ where $\mathfrak{a}$ is an arbitrary ideal in ${ }^{b} \mathbb{C}[X]$, form the closed subsets for a Zariski-topology on ${ }^{b} \mathbb{C}^{n}$. From the assertion (iii) of the Proposition 3.11, we deduce that the inclusion $i: \mathbb{C}^{n} \rightarrow{ }^{b} \mathbb{C}^{n}$ is continuous if both spaces are equipped with the Zariski-topology.

Now, we construct the structure sheaf of ${ }^{b} \mathbb{C}^{n}$. Let $U$ be a nonempty subset of ${ }^{b} \mathbb{C}^{n}$. Define

$$
B(U):=\left\{f \in \mathbb{C}\left[X_{1}, \ldots, X_{n}\right]_{\text {int }} \mid f(x) \in{ }^{b} \mathbb{C} \text { for each } x \in U\right\}
$$

and

$$
S(U):=\left\{f \in \mathbb{C}\left[X_{1}, \ldots, X_{n}\right]_{\text {int }} \mid f(x) \in{ }^{a} \mathbb{C} \text { for each } x \in U\right\}
$$

where ${ }^{a} \mathbb{C}$ denotes the multiplicative set of appreciable elements of ${ }^{b} \mathbb{C}$; that is,

$$
{ }^{a} \mathbb{C}=\left\{x \in{ }^{b} \mathbb{C} \mid x \not 0\right\} .
$$


Trivially, $B(U)$ is an integral domain. We will write $S^{-1} B(U)$ instead of $S^{-1}(U) B(U)$. The correspondence $U \mapsto S^{-1} B(U)$ gives a presheaf of rings over ${ }^{b} \mathbb{C}^{n}$, where $U$ runs over all S-open subsets in ${ }^{b} \mathbb{C}^{n}$. The restriction maps are evident. Such a presheaf is clearly separated. We will denote by $\mathcal{O}_{b} \mathbb{C}^{n}$ its associated sheaf.

Let $p \in{ }^{b} \mathbb{C}^{n}$ and $U$ be an S-open subset of ${ }^{b} \mathbb{C}^{n}$, containing $p$. Hence $U$ also contains $\mu(p)$. We have a canonical morphism

$$
\rho_{U}^{p}: S^{-1} B(U) \rightarrow S^{-1} B(\mu(p))
$$

defined by restriction on $\mu(p)$.

We shall describe the stalks of our structure sheaf

Lemma 3.12 Let $p \in{ }^{b} \mathbb{C}^{n}$ and $g \in{ }^{*} \mathcal{O}\left(\mathbb{C}^{n}\right)$.

i) If $g(\mu(p)) \subset{ }^{b} \mathbb{C}$, there exists an $S$-open subset $V$, containing $p$, such that $g(V) \subset$ ${ }^{b} \mathbb{C}$.

ii) If $g(\mu(p)) \subset{ }^{a} \mathbb{C}$, there exists an $S$-open subset $V$, containing $p$, such that $g(V) \subset$ $a_{\mathbb{C}}$.

Proof The first assertion is an easy consequence of permanence principle. The second assertion is deduced from Theorem 2.1 which asserts that there exists a S-open subset $V$ such that $g$ is S-continuous on $V$ and has a standard part ${ }^{\circ} g$, which is actually holomorphic and ${ }^{\circ} g(z) \neq 0$ for every $z \in{ }^{\circ} V$. In particular, we conclude that $g$ takes appreciable values on $V$.

Applying this lemma for internal polynomials, we prove

Proposition 3.13 For every $p \in{ }^{b} \mathbb{C}^{n}$, there is a natural isomorphism

$$
\mathcal{O}_{b^{n}, p} \rightarrow S^{-1} B(\mu(p))
$$

between the stalk of $\mathcal{O}_{b} \mathbb{C}^{n}$ in $p$ and $S^{-1} B(\mu(p))$.

Proof For each S-open $U$ containing $p$, the morphisms $\rho_{U}^{p}$ are compatible with restriction maps and induce, by taking the inductive limit, the morphism

$$
\lim _{\rightarrow} S^{-1} B(U) \rightarrow S^{-1} B(\mu(p))
$$

which is actually an isomorphism: the permanence principle gives the injectivity and surjectivity is a consequence of Lemma 3.12. 
Remark i) Trivially, the assertion in Lemma 3.12 is false for ${ }^{i} \mathbb{C}$, so we have to distinguish between

$$
\mathfrak{m}_{p}:=\left\{\frac{f}{g} \in S^{-1} B(\mu(p)) \mid f(p) \in{ }^{i} \mathbb{C}^{n}\right\}
$$

the maximal ideal of $S^{-1} B(\mu(p))$ and the ideal of infinitesimal elements

$$
\mathcal{I} n f_{p}:=\left\{\frac{f}{g} \in S^{-1} B(\mu(p)) \mid \exists V, \mathrm{~S}-\text { open }: p \in \mathrm{V}, f(z) \in{ }^{i} \mathbb{C}^{n}, \forall z \in V\right\} .
$$

We have $S^{-1} B(\mu(p)) / \mathcal{I}_{n f_{p}} \cong \mathcal{O}_{\mathbb{C}^{n},{ }^{\circ} p}$.

ii) Let $p \in{ }^{b} \mathbb{C}^{n}$ and $f=\sum_{|\nu| \leq d} b_{\nu}(X-p)^{\nu}$ be an internal polynomial. If $b_{0}$ and $\left|b_{\nu}\right|^{\frac{1}{|\nu|}}$ are bounded for each $\nu$ such that $0<|\nu| \leq d$, then $f \in B(\mu(p))$. This is a consequence of the estimate, used in the proof of 1.4 .

Let $U$ be an S-open. From Theorem 2.1 we deduce that each element $f \in B(U)$ (resp. $f \in S(U)$ ) has a standard part mapping ${ }^{\circ} f \in \mathcal{O}_{\mathbb{C}^{n}}\left({ }^{\circ} U\right)$ (resp. ${ }^{\circ} f \in \mathcal{O}_{\mathbb{C}^{n}}\left({ }^{\circ} U\right),{ }^{\circ} f(x) \neq 0$ for each $x \in{ }^{\circ} U$ ). Using the fact that ${ }^{\circ} U=U \cap \mathbb{C}^{n}$, we get a morphism of presheaves $\mathrm{st}_{\mathrm{U}}: S^{-1} B(U) \rightarrow \mathcal{O}_{\mathbb{C}^{n}}\left(U \cap \mathbb{C}^{n}\right)$, given by $\mathrm{st}_{U}(f / h)={ }^{\circ} f /{ }^{\circ} h$ and which induces a morphism of sheaves

$$
\mathrm{st}_{\mathrm{U}}: \mathcal{O}_{b \mathbb{C}^{n}}(U) \rightarrow \mathcal{O}_{\mathbb{C}^{n}}\left(U \cap \mathbb{C}^{n}\right)
$$

Hence, we have the following proposition

Proposition 3.14 The inclusion map $i: \mathbb{C}^{n} \rightarrow{ }^{b} \mathbb{C}^{n}$ induces an epimorphism of sheaves

$$
\mathcal{O}_{b \mathbb{C}^{n}} \rightarrow i_{*}\left(\mathcal{O}_{\mathbb{C}^{n}}\right)
$$

and so we obtain $i$ as a morphism of ringed spaces.

Proposition 3.15 Let $\mathfrak{a}$ be an ideal of ${ }^{b} \mathbb{C}\left[X_{1}, \ldots, X_{n}\right]$. Then

$$
\operatorname{Supp}\left(\mathcal{O}_{b_{\mathbb{C}^{n}}} / \mathfrak{a} \mathcal{O}_{b_{\mathbb{C}^{n}}}\right)=V(\mathfrak{a}) .
$$

Proof It is clear that $V(\mathfrak{a}) \subset \operatorname{Supp}\left(\mathcal{O}_{b_{\mathbb{C}^{n}}} / \mathfrak{a} \mathcal{O}_{b_{\mathbb{C}^{n}}}\right)$. Conversely, if $x \notin V(\mathfrak{a})$ then there exists $f \in \mathfrak{a}$ such that $f(x) \in{ }^{a} \mathbb{C}$. Since $f$ is S-continuous, we have $f(y) \in{ }^{a} \mathbb{C}$ for each $y \in \mu(x)$, which implies that $1 / f \in \mathcal{O}_{b_{\mathbb{C}^{n}, x}}$ and as a consequence we get $x \notin \operatorname{Supp}\left(\mathcal{O}_{b_{\mathbb{C}^{n}}} / \mathfrak{a} \mathcal{O}_{b_{\mathbb{C}^{n}}}\right)$. 
Now, we are able to define the category of affine *-bounded schemes. An object is given by a locally ringed space $\left({ }^{b} X, \mathcal{O}^{b} X\right)$. The underlying topological space is defined by ${ }^{b} X:=V(\mathfrak{a}) \subset{ }^{b} \mathbb{C}^{n}$ where $\mathfrak{a}$ is an ideal of ${ }^{b} \mathbb{C}\left[X_{1}, \ldots, X_{n}\right]$ and $\mathcal{O}^{b_{X}}:=$ $j_{b}^{-1}\left(\mathcal{O}_{b \mathbb{C}^{n}} / \mathfrak{a} \mathcal{O}_{b \mathbb{C}^{n}}\right)$. The mapping $j_{b:}:{ }^{b} X \rightarrow{ }^{b} \mathbb{C}^{n}$ denotes the inclusion.

Let $\left({ }^{b} Y, \mathcal{O}_{b_{Y}}\right)$ be another object in the category of affine *-bounded schemes where ${ }^{b} Y:=V(\mathfrak{b}) \subset{ }^{b} \mathbb{C}^{m}$. A morphism between $\left({ }^{b} X, \mathcal{O}^{b} X\right)$ and $\left({ }^{b} Y, \mathcal{O}^{b} Y\right)$ is represented by a morphism of ${ }^{b} \mathbb{C}$-algebras,

$$
u_{b}:{ }^{b} \mathbb{C}\left[Y_{1}, \ldots, Y_{m}\right] \rightarrow{ }^{b} \mathbb{C}\left[X_{1}, \ldots, X_{n}\right]
$$

sending the ideal $\mathfrak{b}$ into $\mathfrak{a}$. We demand that the morphism $u_{b}$ should lift to an internal morphism $u: \mathbb{C}\left[Y_{1}, \ldots, Y_{m}\right]_{\text {int }} \rightarrow \mathbb{C}\left[X_{1}, \ldots, X_{n}\right]_{\text {int }}$ defined by $u\left(Y_{i}\right)=g_{i} \in$ ${ }^{b} \mathbb{C}\left[X_{1}, \ldots, X_{n}\right]$ for each $i \in \llbracket 1 . . m \rrbracket$. Then $u_{b}$ induces a morphism of locally ringed spaces between $\left({ }^{b} X, \mathcal{O}_{b} X\right)$ and $\left({ }^{b} Y, \mathcal{O}_{b_{Y}}\right)$ as follows: the morphism $u_{b}$ gives an Scontinuous map $u_{b}^{\prime}:{ }^{b} \mathbb{C}^{n} \rightarrow{ }^{b} \mathbb{C}^{m}$ sending ${ }^{b} X$ into ${ }^{b} Y$, defined by

$$
u_{b}^{\prime}(x)=\left(g_{1}(x), \ldots, g_{m}(x)\right) \text {. }
$$

We will denote by

$$
\alpha_{b}:{ }^{b} X \rightarrow{ }^{b} Y
$$

the restriction of $u_{b}^{\prime}$ on ${ }^{b} X$. The mapping $u_{b}^{\prime}$ defines a morphism of sheaves $\mathcal{O}_{b \mathbb{C}^{m}} \rightarrow$ $\left(u_{b}^{\prime}\right)_{*}\left(\mathcal{O}_{b \mathbb{C}^{n}}\right)$. Finally, we obtain a morphism of sheaves of rings

$$
\mathcal{O}_{b_{Y}} \rightarrow \alpha_{b *}\left(\mathcal{O}_{b_{X}}\right)
$$

induced by $\alpha_{b}$.

We state the main result of this section

Theorem 3.16 There is a natural essentially surjective functor

$$
\text { st : (aff } * \text { bounded schemes) } \longrightarrow \text { (Stein spaces) }
$$

from the category of affine *-bounded schemes to the category of Stein spaces of finite embedding dimension.

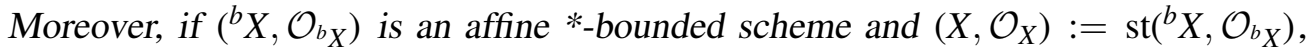
then the inclusion map $i_{b}: X \rightarrow{ }^{b} X$ is a monomorphism.

Proof Let $\left({ }^{b} X, \mathcal{O}_{b_{X}}\right)$ be an object in the category of affine *-bounded schemes, where ${ }^{b} X:=V(\mathfrak{a}) \subset{ }^{b} \mathbb{C}^{n}$. We set

$$
\operatorname{st}\left({ }^{b} X, \mathcal{O}_{b_{X}}\right):=\left(X, \mathcal{O}_{X}\right)=\left(Z\left({ }^{\circ} \mathfrak{a}\right), i^{-1}\left(\mathcal{O}_{\mathbb{C}^{n}} /{ }^{\circ} \mathfrak{a} \mathcal{O}_{\mathbb{C}^{n}}\right)\right)
$$


where $i: Z\left({ }^{\circ} \mathfrak{a}\right) \rightarrow \mathbb{C}^{n}$ denotes the inclusion.

By taking the standard part, the inclusion mapping $i_{b}: X \rightarrow{ }^{b} X$ induces a surjective morphism of sheaves

$$
\mathcal{O}_{{ }^{b} X} \rightarrow i_{b *}\left(\mathcal{O}_{X}\right)
$$

Let $\left({ }^{b} Y, \mathcal{O}_{b_{Y}}\right)$ be another object in the category of affine *-bounded schemes where ${ }^{b} Y:=V(\mathfrak{b}) \subset{ }^{b} \mathbb{C}^{m}$. A morphism $u$ between $\left({ }^{b} X, \mathcal{O}_{b_{X}}\right)$ and $\left({ }^{b} Y, \mathcal{O}^{b} Y\right)$ gives a morphism between the two associated Stein algebras

$$
\operatorname{st}(u): \mathcal{O}\left(\mathbb{C}^{m}\right) / \overline{{ }^{\circ} \mathfrak{b}} \longrightarrow \mathcal{O}\left(\mathbb{C}^{n}\right) /{ }^{\circ} \mathfrak{a} .
$$

Such a morphism induces a morphism between the Stein spaces $\left(X, \mathcal{O}_{X}\right)$ and $\left(Y, \mathcal{O}_{Y}\right)$.

It remains to prove that the functor "st" is essentially surjective. Let $X$ be a Stein space of finite embedding dimension. Then there exists an ideal $\mathfrak{a} \subset \mathcal{O}\left(\mathbb{C}^{n}\right)$, generated by a family of entire holomorphic functions $\left(g_{i}\right)_{i \in I}$, such that $X=Z(\mathfrak{a})$ and $\mathcal{O}_{X}=$ $\mathcal{O}_{\mathbb{C}^{n}} / \mathfrak{a} \mathcal{O}_{\mathbb{C}^{n}}$. For each $i \in I$, let $h_{i}$ be a bounded internal polynomial such that ${ }^{\circ} h_{i}=g_{i}$. We put $\mathfrak{a}_{b}=\sum_{i \in I} h_{i}{ }^{b} \mathbb{C}\left[X_{1}, \ldots, X_{n}\right],{ }^{b} X=V\left(\mathfrak{a}_{b}\right)$ and $\mathcal{O}_{b_{X}}:=\mathcal{O}_{b} \mathbb{C}^{n} / \mathfrak{a}_{b} \mathcal{O}_{b}$. Clearly, we obtain $\operatorname{st}\left({ }^{b} X, \mathcal{O}_{b_{X}}\right):=\left(X, \mathcal{O}_{X}\right)$.

Theorem 3.17 There is a natural functor between the category of affine schemes of finite type over $\mathbb{C}$ and the category of affine *-bounded schemes.

Proof Each polynomial over $\mathbb{C}$ can be regarded as an internal bounded polynomial. We consider the functor which associates to each algebra of finite type over $\mathbb{C}$, say $A=$ $\mathbb{C}\left[X_{1}, \ldots, X_{n}\right] /\left(f_{1}, \ldots, f_{q}\right)$, the affine ${ }^{*}$-bounded scheme ${ }^{b} X:={ }^{*} Z\left(f_{1}, \ldots, f_{q}\right) \cap{ }^{b} \mathbb{C}^{n}$ and the structural sheaf $\mathcal{O}_{b \mathbb{C}^{n}} / \sum_{i=1}^{q} f_{i} \mathcal{O}_{b} \mathbb{C}^{n}$. This construction is functorial and gives us our functor. -

\section{Global *-bounded schemes and the standard part functor}

\subsection{The basic functors}

In this section, we want to define a category of global schemes where the affine pieces are those described in Section 3. This is done via locally ringed spaces and we immediately obtain two new categories, the category of general $*_{\text {-schemes denoted by }}$

$$
(*-\mathrm{sch})
$$


and

$$
(* b-\mathrm{sch})
$$

that of so-called *-bounded schemes which are more related to complex geometry. The first one is of a very general nature (that is, a category of schemes over ${ }^{*} \mathbb{Z}$ ) whilst the second one is a category of schemes over the ring ${ }^{b} \mathbb{C}$ of bounded complex numbers. We note that the structure sheaf of any *-bounded scheme $X$ contains an intrinsic ideal sheaf $\mathcal{I} n f_{X}$ of infinitesimal sections which is locally described in Section 3.2.

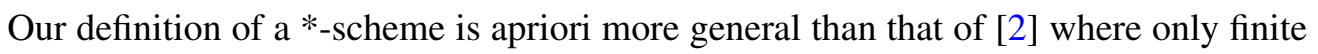
coverings by affine pieces are allowed. In order to define the notion of an internal subspace, we would have to make restrictions on the cardinality of coverings, since being internal is a global property.

We have an evident functor "associated *-scheme" from the category of algebraic $\mathbb{C}$ schemes, locally of finite type

$$
(\mathbb{C}-\text { schemes lft }) \longrightarrow(* b-\mathrm{sch})
$$

which associates to a locally algebraic $\mathbb{C}$-scheme $X$ its *-bounded version ${ }^{b} X$ and similar at the level of morphisms.

Example 4.1 If $\mathbb{P}_{\mathbb{C}}^{n}$ denotes the n-dimensional projective space over the complex numbers, then ${ }^{b} \mathbb{P}_{\mathbb{C}}^{n}$ can be constructed as the quotient of ${ }^{b} \mathbb{C}^{n+1} \backslash \mu(0)$ by the action of the multiplicative system of all appreciable complex numbers. Note that the natural map ${ }^{b} \mathbb{P}_{\mathbb{C}}^{n} \rightarrow{ }^{*} \mathbb{P}_{\mathbb{C}}^{n}$ is bijective ( $n$ is standard).

Recall that to every affine bounded scheme we associated in Section 3 a Stein complex space (of finite embedding dimension) in a functorial way. By a straightforward generalisation, we obtain a functor

$$
\text { st }:(* b-\mathrm{sch}) \longrightarrow \text { (complex spaces) }
$$

which we call the standard part functor. We sometimes write also ${ }^{\circ} X=\operatorname{st}(X)$ for the standard part of a bounded scheme $X$. We may regard ${ }^{\circ} X$ as the "subspace" of $X$, defined by the ideal sheaf $\mathcal{I} n f_{X}$ all infinitesimal sections and so we have a natural morphism of ringed spaces

$$
{ }^{\circ} X \rightarrow X
$$

The conormal sheaf of this embedding is of particular interest, since it gives us a non standard interpretation of classical differential forms on ${ }^{\circ} X$ (see Section 6). 
Theorem 4.2 The standard part functor "st" possesses a left adjoint functor ${ }^{b}$ : (complex spaces) $\rightarrow(* \mathrm{~b}-\mathrm{sch})$ which associates to every complex space $X$ a natural *-bounded scheme ${ }^{b} X$ with locally no nontrivial infinitesimal elements. Moreover, the adjunction morphism $i d \rightarrow$ st $\circ\left(^{(}\right)$is an isomorphism.

Proof We first describe the functor $X \mapsto{ }^{b} X$ locally; that is, for $X$ a finite dimensional Stein spaces (as in Section 2). As we have seen, there is an affine *-bounded scheme $\mathfrak{X}$ such that $X \cong{ }^{s t} \mathfrak{X}$. We can now make a natural minimal choice of $\mathfrak{X}$ where there are no nontrivial infinitesimal elements in the local rings. In this case, the natural homomorphism $\mathcal{O}_{\mathfrak{X}} \rightarrow\left(i_{b}\right)_{*} \mathcal{O}_{X}$ will be an isomorphism. This construction is evidently functorial in $X$, so it globalizes to complex spaces and we get our desired functor. Intuitively speaking, we just enlarge $X$ (locally) by its bounded points and conserve at the same time its structure sheaf.

In order to verify the adjunction property, let $\mathfrak{X}$ be a *-bounded scheme and $Y$ a complex space. By applying the functor "st", we get a functorial map

$$
\operatorname{Hom}\left({ }^{b} Y, \mathfrak{X}\right) \longrightarrow \operatorname{Hom}(Y, \operatorname{st}(\mathfrak{X}))
$$

which is immediately seen to be bijective, since there are locally no nontrivial infinitesimal elements in the structure sheaf of ${ }^{b} Y$. The last assertion is an obvious consequence of our construction.

\subsection{DG- algebra resolutions of complex spaces via bounded polynomial algebras}

Sometimes it is useful to replace global (complex) spaces by local simplicial ones. In the case of algebras (over the rationals), it is sufficient to deal with differential graded ones.

Let us now consider a differential graded algebra $R=\oplus_{k \leq 0} R_{k}$ with differential $s$ such that $R_{0}$ is an algebra of bounded polynomials and $R$ is freely generated over $R_{0}$. Then, we can associate to it a Stein algebra in the following way: We may write $R_{0}={ }^{b} \mathbb{C}\left[T_{1}, \ldots, T_{n}\right]$. Then $\mathcal{S T}\left(\operatorname{Coker}\left(R_{-1} \rightarrow R_{0}\right)\right)$ is, by 2.17 , a Stein algebra. This definition is clearly functorial. Since it is evidently possible to construct DG-resolutions of that type for a given Stein algebra of finite embedding dimension, we obtain

Proposition 4.3 The above defined functor from the category of free bounded DGalgebras which are exact in negative degrees, to the category of Stein algebras of finite embedding dimension is essentially surjective. 
We generalize this fact to the simplicial case. Let $M$ be a totally ordered set and $\mathcal{N}$ the category of non empty finite subsets of $M$. An object $\alpha$ of $\mathcal{N}$ (that is, a non empty finite subset of $M$ ) is called a simplex. Its dimension is, by definition, $\operatorname{card}(\alpha)-1$. The set $\operatorname{Mor}_{\mathcal{N}}(\alpha, \beta)$ is of cardinality $\leq 1$ corresponding to the condition if $\alpha \subset \beta$ or not. A simplicial object in a category $\mathcal{C}$ is a contravariant functor $\mathcal{N} \rightarrow \mathcal{C}$. The category of all these functors (or $\mathcal{N}$-objects) is denoted by $\mathcal{C}_{\mathcal{N}}$. We can show

Proposition 4.4 The functor "standard part in degree zero cohomology" that is defined above, from the category of free bounded DG- $\mathcal{N}$-algebras which are exact in negative degrees, to the category of Stein $\mathcal{N}$-algebras of simplicialwise finite embedding dimension, is essentially surjective.

For a sketch of the proof, given a simplicial Stein algebra $A$ (simplicialwise of finite embedding dimension), we proceed in the usual way by induction on the dimension of simplicies to construct a free DG-algebra resolution $R_{\alpha}$ such that ${ }^{\circ} \mathrm{H}^{0}\left(R_{\alpha}\right)=A_{\alpha}$. If $\operatorname{dim} \alpha=0$, this has been done. For $\operatorname{dim} \alpha>0$ we already know the values of the differential on all free generators which come from strictly lower dimensional simplicies, so that we just add some new generators and construct the differential $s$ by descending induction on the degree of generators in order to obtain exactness in negative degrees and such that $\mathcal{S T}\left(\mathrm{H}^{0}\left(R_{\alpha}\right)\right)=A_{\alpha}$. -

We want to describe another more subtle construction of such resolutions, by imposing additional assumptions on the resolution type. For this, we fix a complex space $X$, an embedding $X$. $\hookrightarrow P$. into a simplicial (free) polydisc $P$. and a free simplicial DGalgebra resolution $(\mathcal{R}, s)$ of $\mathcal{O}_{X}$. of the form $\mathcal{R}=\mathcal{O}_{P}$. $\left[e_{i}\right]_{i \in I}$ with free generators $e_{i}$ of strictly negative degrees. The graded simplicial algebra $\mathcal{R}$ admits an evident lifting to the category of simplicial affine bounded algebras. But we cannot extend directly the identity $s^{2}=0$ to this algebra. Nevertheless, we will obtain it on a suitable (even maximal) affine subspace $\mathfrak{Z}$. $\hookrightarrow{ }^{b} P$., after fixing an infinite natural number $N$. The definition of $\mathfrak{Z}$. is the following: we first lift $s$ to bounded derivation $s_{N}$ on ${ }^{b} \mathcal{R}=\mathcal{O}_{{ }^{b} \text {. }}\left[e_{i}\right]_{i \in I}$. Then $s_{N}^{2}\left(e_{i}\right)$ is infinitesimal by construction. Dividing out these infinitesimal polynomials (over each simplex), we obtain a subspace $\mathfrak{Z}$. $\hookrightarrow{ }^{b} P$. over which the class of $s_{N}^{2}$ is zero. In other words, we get a simplicial DG-algebra in the category of bounded algebras, conserving all original free generators of negative degree (and not adding any further ones).

Remark The construction above is inspired by deformation theory of complex spaces: describing all small deformations of $X$ by varying the differential of a fixed DGalgebra resolution of $\mathcal{O}_{X}$. Moreover, our construction leads us to an obvious definition 
of what should be a deformation of $X$ over local *-bounded algebras, for example algebras of the form ${ }^{b} \mathbb{C} / \mathfrak{a}$ where $\mathfrak{a}$ is an ideal generated by some infinitesimal complex numbers. Such nonstandard or Leibniz deformations give an interesting alternative approach to deformation theory where the meaning of "infinitesimal deformation" becomes a metric one.

\subsection{Special features of the standard part construction}

We give some applications for the passage from the holomorphic to the *-bounded algebraic context.

Let $H$ be a standard hyperplane in the ${ }^{*}$-scheme ${ }^{*} \mathbb{P}_{\mathbb{C}}^{n}$. Then the natural inclusion $* \mathbb{C}^{n} \hookrightarrow * \mathbb{P}_{\mathbb{C}}^{n}$ induced by $H$, allows us to define the notion of a bounded point in ${ }^{*} \mathbb{P}_{\mathbb{C}}^{n} \backslash H$. The following theorem shows that we can "compactify" analytic subsets of $\mathbb{C}^{n}$ in a (non-canonical) way to projective $*_{\text {-schemes }}$

Theorem 4.5 Let $X \subset \mathbb{C}^{n}$ be an analytic subset. Then there is a standard hyperplane $H \subset{ }^{*} \mathbb{P}_{\mathbb{C}}^{n}$ and a ${ }^{*}$-projective subvariety $\mathfrak{X} \subset{ }^{*} \mathbb{P}_{\mathbb{C}}^{n}$ such that $X$ is the standard part of $\mathfrak{X} \backslash H$.

Proof Let $X$ be the zero set of a countable family $\left(f_{j}\right)_{j \in \mathbb{N}}$ of holomorphic functions on $\mathbb{C}^{n}$. We fix infinite natural numbers $N, M$ and a hyperfinite family $\left(F_{j}\right)_{0 \leq j \leq M}$ of $*_{-}$ bounded polynomials of degree $\leq N$ such that. $f_{j}=\operatorname{st}\left(F_{j}\right)$ for $j$ finite. We may assume that $F_{j}$ is infinitesimal for $j$ infinite. Next, we homogenize each $F_{j}$ to a homogeneous internal (but in general not $*$-bounded !) polynomial $\tilde{F}_{j}$ of degree $\leq N$ in $n+1$ variables. Let $\mathfrak{X} \subset{ }^{*} \mathbb{P}_{\mathbb{C}}^{n}$ be defined by the $\tilde{F}_{j}$ 's. Then, by our construction, $\mathfrak{X}$ has the desired property.

Theorem 4.6 Let $X$ be an affine algebraic $\mathbb{C}$-scheme such that $\mathrm{H}^{2}\left(X^{a n}, \mathbb{Z}\right)=0$. Then every holomorphic map $f: X^{a n} \rightarrow \mathbb{P}_{\mathbb{C}}^{n}$ from $X^{a n}$ to a projective space is the standard part of a ${ }^{*}$-bounded morphism $F:{ }^{b} X \rightarrow^{b} \mathbb{P}_{\mathbb{C}}^{n}$.

Proof By our cohomological assumption, we can lift $f$ to a holomorphic map $\tilde{f}$ : $X^{a n} \rightarrow \mathbb{C}^{n+1} \backslash\{0\}$. By the results of section 2.5, the map $\tilde{f}$ is the standard part of a $*_{-}$ bounded map $F:{ }^{b} X \rightarrow{ }^{b} \mathbb{C}^{n+1}$ which avoids necessarily the halo of $\{0\}$. By passing to the quotient (as in 4.1), we get our result. 
Example 4.7 Let $f: \mathbb{C} \rightarrow \mathbb{P}_{\mathbb{C}}^{n}$ be a holomorphic map. By fixing an infinite natural number $N$, we may extend $f$ to a morphism $f_{N}: b \mathbb{C} \rightarrow^{b} \mathbb{P}_{\mathbb{C}}^{n}$ of $*$-bounded schemes such that $\operatorname{st}\left(f_{N}\right)=f$. Moreover, applying homogenization, we can lift $f_{N}$ to a *algebraic map $\tilde{f_{N}}:{ }^{*} \mathbb{P}^{1} \rightarrow{ }^{*} \mathbb{P}_{\mathbb{C}}^{n}$ which will be a ${ }^{*}$-bounded morphism if and only if $f$ is algebraic. In fact, we can define $\tilde{f_{N}}:{ }^{b} \mathbb{P}^{1} \rightarrow{ }^{b} \mathbb{P}_{\mathbb{C}}^{n}$ just as a map, but it will not be continuous at the point $(1: 0)$ if $f$ is not algebraic.

We close this section by mentioning a simple fact concerning the standard points of internal subspaces of nonstandard complex spaces

Theorem 4.8 Let $X$ be a complex space and $\mathfrak{Y} \subset{ }^{*} X$ an internal complex subspace of (finite) codimension $k$. Then the intersection $\mathfrak{Y} \cap X$ is contained in a countable union of complex subspaces $Y_{n}, n \in \mathbb{N}$, of $X$ of codimension $k$. The same conclusion holds in the algebraic context.

For the proof, we note that $\mathfrak{Y}={ }^{*} Y_{I}$ for $\left(Y_{i}\right)_{i \in I}$ a system of complex subspaces of codimension $k$. If we put $Y=\cup_{i} Y_{i}$, then $\mathfrak{Y} \subset{ }^{*} Y$ and $\mathfrak{Y} \cap X \subset Y$.

\section{The Nullstellensatz and nonstandard generic points for com- plex spaces}

In this section, we prove in particular that any maximal ideal of a Stein algebra $A$ is the vanishing ideal of an eventually nonstandard point. Moreover, we can show that any prime ideal of $A$ is determined by its nonstandard zero set if and only if it satisfies a Hilbert Nullstellensatz. We give a large class of examples of prime ideals which are not determined by their nonstandard zero set and with necessarily empty standard zero set. Closed ideals in Stein algebras are extensively treated in Forster's paper [6].

Our notion of a nonstandard zero set of an ideal allows us to interpret generic points of irreducible complex spaces in a natural geometric way.

\subsection{Nonstandard zeros of holomorphic functions and the Nullstellensatz}

Let $X$ be a Stein complex space and $A:=\Gamma\left(X, \mathcal{O}_{X}\right)$ its algebra of global holomorphic sections. For any ideal $I \subset A$, we define the nonstandard zero set of $I$ by

$$
\mathrm{V}(I):=\left\{x \in{ }^{*} X \mid{ }^{*} f(x)=0, \forall f \in I\right\} .
$$


Forster proved that the (standard) zero set of a proper closed ideal in a Stein algebra is non empty. Siu[17] showed that the closedness is essential and gave an example of an ideal whose variety is empty. We prove that the nonstandard zero set of any proper ideal is nonempty. This indicates that our definition is an adequate notion of a zero set in the context of Stein algebras. Let $I \subsetneq A$ be a proper ideal. We define the binary relation $P$ on $X \times I$ by $P<x, f>$ if $f(x)=0$. The relation $P$ is concurrent: if $f_{1}, \ldots, f_{r} \in I$ then there exists $x \in X$ such that $f_{i}(x)=0$ for each $i=1, \ldots, r$. Since ${ }^{*} X$ is an enlargement of $X$, there exists $x \in{ }^{*} X$ such that ${ }^{*} f(x)=0$ for all $f \in I$ and hence $\mathrm{V}(I) \neq \emptyset$ (see Appendix B: Concurrence or Saturation Principle).

There is also the dual construction: Let $M \subset{ }^{*} X$ be any subset. We put

$$
\operatorname{Id}(M):=\left\{f \in A \mid{ }^{*} f(x)=0, \forall x \in M\right\}
$$

the nonstandard ideal of $M$ in $A$. These constructions transform an inclusion into the opposite one. Moreover, we have the following rules: Let $\left(I_{r}\right)_{r \in R}$ be a family of ideals in $A$ and $\left(M_{S}\right)_{s \in S}$ a family of subsets of ${ }^{*} X$. Then

$$
\mathrm{V}\left(\sum_{r \in R} I_{r}\right)=\cap_{r \in R} \mathrm{~V}\left(I_{r}\right),
$$

and

$$
\mathrm{V}\left(\cap_{r \in R} I_{r}\right)=\mathrm{V}\left(\Pi_{r \in R} I_{r}\right)=\cup_{r \in R} \mathrm{~V}\left(I_{r}\right)
$$

if $R$ is finite, dually, for " $I d$ "

$$
\operatorname{Id}\left(\cup_{s \in S} M_{s}\right)=\cap_{s \in S} \operatorname{Id}\left(M_{s}\right),
$$

and

$$
\operatorname{Id}\left(\cap_{s \in S} M_{s}\right) \supset \Sigma_{s \in S} \operatorname{Id}\left(M_{s}\right) .
$$

Moreover, for any subset $M \subset{ }^{*} X$ and any ideal $I \subset A$, we have clearly

$$
\begin{aligned}
& \mathrm{V}(\operatorname{Id}(M)) \supset M, \\
& \operatorname{Id}(\operatorname{V}(I)) \supset \sqrt{I} .
\end{aligned}
$$

More precisely, for the first one, we show

Proposition 5.1 We have $\mathrm{V}(\operatorname{Id}(M))=\cap_{Y} \mathrm{~V}(\operatorname{Id}(Y))$, where the intersection is taken over all $Y \subset X$ closed analytic subsets such that $M \subset{ }^{*} Y$.

Proof If $Y \subset X$ is analytic, we get $\operatorname{Id}\left({ }^{*} Y\right)=\operatorname{Id}(Y)$ and therefore $\mathrm{V}\left(\operatorname{Id}\left({ }^{*} Y\right)\right)=$ $\mathrm{V}(\operatorname{Id}(Y)) \supset{ }^{*} Y$. We note that $\operatorname{Id}(M)=\Sigma_{Y} \operatorname{Id}(Y)$, where the sum is taken over all $Y \subset X$ analytic such that $M \subset{ }^{*} Y$, which gives us the desired identity. - 
We want to show that every prime ideal of $A$ is the ideal of a (nonstandard) point of $X$ if and only if it satisfies a Hilbert Nullstellensatz.

Theorem 5.2 i) Let $\mathfrak{m}$ be a maximal or a minimal prime ideal of $A$, then $\mathfrak{m}$ is of the form $\operatorname{Id}(\{x\})$,

ii) Let $\mathfrak{p}$ be a prime ideal of $A$, then $\mathfrak{p}$ is of the form $\operatorname{Id}(\{x\})$, if and only if it satisfies the Nullstellensatz; that is, $\operatorname{Id}(\mathrm{V}(\mathfrak{p}))=\mathfrak{p}$.

Proof i) We first treat the case of a maximal ideal. By the Nullstellensatz, we have

$$
\forall n \in \mathbb{N}, \forall f_{1}, \ldots, f_{n} \in \mathfrak{m} \Rightarrow \exists y \in X: f_{i}(y)=0 \forall i, 1 \leq i \leq n .
$$

By the concurrence principle (see the Appendix B: Concurrence or Saturation Principle), we conclude that there is a point $x \in{ }^{*} X$ such that $\mathfrak{m} \subseteq \operatorname{Id}(\{x\})$. Since $\mathfrak{m}$ is maximal, we have equality.

ii) First, let $\mathfrak{p}$ be of the form $\operatorname{Id}(\{x\})$. Then $\operatorname{V(p}) \supset\{x\}$ and so $\operatorname{Id}(\mathrm{V}(\mathfrak{p})) \subset \operatorname{Id}(\{x\})=$ $\mathfrak{p}$, which finally gives equality. For the converse, we note that it suffices to show

$$
\forall n \in \mathbb{N}, \forall f_{1}, \ldots, f_{n} \in \mathfrak{p}, \forall g_{1}, \ldots, g_{n} \notin \mathfrak{p} \Rightarrow \exists y \in X: f_{i}(y)=0 \forall i, g_{1}(y) \cdot \ldots \cdot g_{n}(y) \neq 0
$$

and since $g_{1} \cdot \ldots \cdot g_{n} \notin \mathfrak{p}$, we may take $n=1$ for the $g_{i}^{\prime} s$ for the verification of this implication. By applying the concurrence principle, we obtain

$$
\exists x \in{ }^{*} X: \forall f \in \mathfrak{p}, \forall g \notin \mathfrak{p} \Rightarrow{ }^{*} f(x)=0,{ }^{*} g(x) \neq 0
$$

which means precisely that $\mathfrak{p}=\operatorname{Id}_{X}(x)$. So let now be $f_{1}, \ldots, f_{n} \in \mathfrak{p}$ and $g \notin \mathfrak{p}$. Assume that for the usual zero sets we have $\mathrm{Z}\left(f_{1}, \ldots, f_{n}\right) \subset \mathrm{Z}(g)$. Then, we get also $\mathrm{V}\left(f_{1}, \ldots, f_{n}\right) \subset \mathrm{V}(g)$ and so for the corresponding ideals $\operatorname{Id}\left(\mathrm{V}\left(f_{1}, \ldots, f_{n}\right)\right) \supset \operatorname{Id}(\mathrm{V}(g)) \ni$ g. But, $\operatorname{Id}\left(\mathrm{V}\left(f_{1}, \ldots, f_{n}\right)\right) \subset \operatorname{Id}(\mathrm{V}(\mathfrak{p}))=\mathfrak{p}$ and we would obtain $g \in \mathfrak{p}$, which is a contradiction. This shows (ii).

iii) It remains to show that any minimal prime ideal is that of a point. Let $\mathfrak{p}$ be a prime ideal. In contrast to the formula above, the following implication is always true

$$
\forall n \in \mathbb{N}, \forall g_{1}, \ldots, g_{n} \notin \mathfrak{p} \Rightarrow \exists y \in X: g_{1}(y) \cdot \ldots \cdot g_{n}(y) \neq 0
$$

since it is true for $n=1$ and $\mathfrak{p}$ is prime. By the concurrence principle, we conclude that there is a point $x \in{ }^{*} X$ such that

$$
\forall g \notin \mathfrak{p} \Rightarrow g(x) \neq 0
$$

which means $\mathfrak{p} \supset \operatorname{Id}_{X}(x)$, so finally $\mathfrak{p}=\operatorname{Id}_{X}(x)$ by minimality of $\mathfrak{p}$. 
Example 5.3 i) Regarding the inclusion $A \hookrightarrow{ }^{*} A$, we obtain certain prime ideals of $A$ by intersecting just with those of ${ }^{*} A$. In particular, every internal prime ideal of ${ }^{*} A$ gives us one of $A$ (for example, fixing $\left(\mathfrak{p}_{i}\right)_{i}$, a countable sequence of closed prime ideals of $A$ ).

ii) Assume $A$ to be an integral domain and that we have an "order function" $\omega$ : $A \backslash\{0\} \rightarrow{ }^{*} \mathbb{N}$; that is, $\omega$ satisfies $\omega(f g)=\omega(f)+\omega(g)$ and $\omega(f+g) \geq$ $\inf \{\omega(f), \omega(g)\}$ as well as $\omega(1)=0$. Then the subset of $A$ formed by zero and all elements of infinite $\omega$-order is a prime ideal of $A$.

iii) Combining the constructions of (i) and (ii), we can immediately construct explicitly many prime ideals of the ring of entire holomorphic functions on $\mathbb{C}^{n}$ which are not ideals of nonstandard points.

We can determine the possible residue fields of a maximal ideal in a Stein algebra

Theorem 5.4 Let $A$ be a Stein algebra (of finite embedding dimension) and $\mathfrak{m}=$ $\operatorname{Id}(x)$ any maximal ideal of $A$. We suppose that the used ultrafilter is $\delta$-stable (see Appendix $A: \delta$-stable ultrafilters). Then the evaluation map $\chi: A / \mathfrak{m} \rightarrow{ }^{*} \mathbb{C}$ in $\{x\}$ is an isomorphism of fields if $\{x\}$ is an infinite point and $\operatorname{Im}(\chi)=\mathbb{C}$ if $\{x\}$ is bounded. In the last case, $\{x\}$ is standard and so $\mathfrak{m}$ is a closed ideal.

Proof We may assume that $A=\Gamma\left(\mathbb{C}^{n}, \mathcal{O}_{\mathbb{C}^{n}}\right)$. If $\{x\}$ is an infinite point, then at least one coordinate of $\{x\}$, say $\left\{x_{k}\right\}$, is infinite. By projecting to this coordinate, we may take first $n=1$. But in this case, the point can be represented (using $\delta$-stability) by a sequence in $\mathbb{C}$ which tends to $\infty$. Clearly, here the residue field must be ${ }^{*} \mathbb{C}$, by classical function theory in one complex variable. In this way, we get homomorphisms of fields $\kappa\left(x_{k}\right) \rightarrow \kappa(x) \rightarrow{ }^{*} \mathbb{C}$, where the second arrow is induced by evaluation in $\{x\}$. The composition is, by construction, evaluation in ${ }^{*} \mathbb{C}$ and therefore bijective.

If the point $\{x\}$ is bounded in ${ }^{*} \mathbb{C}^{n}$, it has a standard part $a$. Since every holomorphic function which vanishes in $\{x\}$, must also vanish in $a$, we obtain, by maximality of $\mathfrak{m}$, our result.

We do not know if the $\delta$-stability of the ultrafilter is essential for the above result to hold. Therefore, we assume this property for the rest of this section.

\subsection{On the spectrum of a Stein algebra}

Here is the main result of this section 
Theorem 5.5 Let $X$ be a Stein complex space. Then the image of the natural map

$$
\operatorname{Id}_{X}:{ }^{*} X \longrightarrow \operatorname{Spec}\left(\Gamma\left(X, \mathcal{O}_{X}\right)\right)
$$

which associates to a point $x \in{ }^{*} X$ its ideal $\operatorname{Id}(\{x\})$, consists of all prime ideals satisfying the Nullstellensatz. Moreover, this image contains all maximal, minimal and all closed prime ideals of $\Gamma\left(X, \mathcal{O}_{X}\right)$.

Proof First, we remark that this map is well-defined, by considering the evaluation homomorphism $\Gamma\left(X, \mathcal{O}_{X}\right) \rightarrow{ }^{*} \mathbb{C}$, given by a point $x \in{ }^{*} X$. Let $\mathfrak{p}$ be a prime ideal in $\Gamma\left(X, \mathcal{O}_{X}\right)$. By the results of the last subsection, we only need to treat the case of closed prime ideals. But such a prime ideal satisfies the usual Nullstellensatz (see [6]) and so in particular the nonstandard one; that is, $\operatorname{Id}(\mathrm{V}(\mathfrak{p}))=\mathfrak{p}$. Again, 5.2 gives us our statement.

Corollary 5.6 Let $X$ be an irreducible Stein complex space. Then there exists a point $x \in{ }^{*} X$ such that the evaluation in $x$

$$
\chi_{x}: \Gamma\left(X, \mathcal{O}_{X}\right) \longrightarrow{ }^{*} \mathbb{C}
$$

is injective. For the field of meromorphic functions, we get

$$
\mathcal{M}(X) \longrightarrow{ }^{*} \mathbb{C},
$$

the induced homomorphism of fields.

Now, we come to study some topological properties of the map $\operatorname{Id}_{\mathrm{X}}$. One can define a topology $\mathcal{T}$ on ${ }^{*} X$ where $V(I)$ are closed sets for this topology (see section 5.1). For each $f \in A=\Gamma\left(X, \mathcal{O}_{X}\right)$, the open set

$$
\mathcal{D}(f)=\left\{x \in{ }^{*} X,{ }^{*} f(x) \neq 0\right\}={ }^{*}\{x \in X, f(x) \neq 0\}={ }^{*} D(f)
$$

is a distinguished open set, the family of distinguished open sets is a basic for the topology $\mathcal{T}$. Hence $\mathcal{T}$ is the S-topology on ${ }^{*} X$ when $X$ is equipped with the Zariski topology where $\{D(f), f \in A\}$ forms an open base, called the S-Zariski topology on ${ }^{*} X$ (see Appendix C Nonstandard Topologies).

Proposition 5.7 Let $X$ be a Stein complex space. Then the natural map

$$
\operatorname{Id}_{X}:{ }^{*} X \longrightarrow \operatorname{Spec}\left(\Gamma\left(X, \mathcal{O}_{X}\right)\right)
$$

is continuous for both the $S$-Zariski topology on ${ }^{*} X$ and the Zariski topology on $\operatorname{Spec}\left(\Gamma\left(X, \mathcal{O}_{X}\right)\right)$. 
Proof For each $x \in{ }^{*} X$ and $f \in \operatorname{Spec}\left(\Gamma\left(X, \mathcal{O}_{X}\right)\right)$, we have $f \in \operatorname{Id}_{X}(x)$ if and only if, ${ }^{*} f(x)=0$. Hence, we get $\operatorname{Id}_{X}{ }^{-1}(D(f))=\mathcal{D}(f)$, which shows that our map is continuous.

Definition 5.8 Let $X$ be a complex space and $x \in{ }^{*} X$ a point. The Zariski closure $\bar{x}$ of $x$ in $X$ is the smallest analytic subset $Y$ of $X$ such that $x \in{ }^{*} V$ for every Zariskiopen $V \subset Y$. If $Y \subset X$ is an analytic subset and $x \in{ }^{*} Y$, we call $x$ a generic point of $Y$ if $\bar{x}=Y$.

Remark i) The Zariski closure $\bar{x}$ of $x$ in $X$ is always irreducible.

ii) Note that $\bar{x}$ may be sometimes the empty set, for example if $X=\mathbb{C}$ and $x \in{ }^{*} \mathbb{C}$ is given be a discrete sequence converging to infinity.

Theorem 5.9 Let $X$ be an irreducible complex space. Then $X$ has always a generic point. Moreover, for every standard point $x \in X$, there is a generic point of $X$ in the halo of $x$ (for the usual topology).

Proof Let $U_{1}, \ldots, U_{n}$ be a finite family of non-empty Zariski open subsets of $X$. Then, by the irreducibility of $X$, the intersection $U_{1} \cap \ldots \cap U_{n}$ is also non-empty. The concurrence principle allows us to conclude that there is a point $\eta \in{ }^{*} X$ such that $\eta \in{ }^{*} U$ for every non-empty Zariski open subset $U$ of $X$. This point will be generic for $X$ : Let $Y \subset X$ be any strictly smaller analytic subset such that $\eta \in{ }^{*} Y$. Since we have $\eta \in{ }^{*}(X \backslash Y)$ too, we get a contradiction. This shows the first part of 5.9.

We refine slightly our argument in order to obtain the second part. Let $x$ be a standard point of $X$. We decompose $X$ locally around $x \in X$ into irreducible components $X_{1} \cup \ldots \cup X_{r}$ and fix one of them, say $X_{1}$. In $X_{1}$, we consider the subsets of the form $V \backslash Y$ where $V$ is a (usual) open neighborhood of $x$ in $X_{1}$ and $Y \subset V$ a strictly smaller closed analytic subset, defined by finitely many holomorphic functions on $V$. Clearly, every finite intersection of such subsets is non-empty. By the concurrence principle, there is a point $\eta \in{ }^{*}(V \backslash Y)$ for every one of our subsets $V \backslash Y$. Evidently, $\eta$ is bounded and its standard part is $x$ by construction. We claim that $\eta$ is a generic point for $X$. Let $Z \subset X$ be a strictly smaller analytic subset. By irreducibility and purity of dimension of $X$, we cannot have $Z=X_{1}$ locally around $x$. So $Z$ is strictly smaller than $X_{1}$ in some neighborhood of $x$ too. We therefore get $\eta \in{ }^{*}(X \backslash Z)$ which means that $\eta$ is a generic point for $X$.

Remark We should mention, in this context, the paper of G.Wallet [19] in which he shows algebraic versions of the existence of nonstandard generic points by a more direct method. The case of complex space space germs has already been treated by A.Robinson in [16]. 


\section{Differential forms seen in a modern nonstandard way}

In this section, we show how differential forms find a natural description in the context of our *-bounded algebras. We prove in particular that they coincide (in all relevant cases) with the associated analytic ones.

Let $D_{n}$ be the subring of the internal polynomials in $2 n$ variables $\mathbb{C}[X, d X]_{\text {int }}=$ $\mathbb{C}\left[X_{1}, \ldots, X_{n}, d X_{1}, \ldots, d X_{n}\right]_{\text {int }}$ defined by

$$
D_{n}:=\left\{P \in \mathbb{C}[X, d X]_{\text {int }} \mid P(x, \xi) \in{ }^{b} \mathbb{C} \text { for each }(x, \xi) \in{ }^{b} \mathbb{C}^{n} \times{ }^{i} \mathbb{C}^{n}\right\} .
$$

By Theorem 2.1, it is immediate that the ring $D_{n}$ is invariant under derivations. Furthermore, each $P \in D_{n}$ can be written as

$$
P(X, d X)=P_{0}(X)+\sum_{i=1}^{n} Q_{i}(X, d X) d X_{i}
$$

where $P_{0}(X)=P(X, 0) \in{ }^{b} \mathbb{C}\left[X_{1}, \ldots, X_{n}\right]$ and $Q_{i} \in D_{n}$, since $Q_{i}(x, \xi)=\int_{0}^{1} \frac{\partial P}{\partial \xi_{i}}(x, t \xi) d t$. More generally, let $A={ }^{b} \mathbb{C}[X] / \mathfrak{a}$ be a *-bounded algebra, using the abbreviation $X=\left(X_{1}, \ldots, X_{n}\right)$. We define an infinitesimal version of the diagonal algebra of $A$ by setting

$$
D(A):=D_{n} / \mathfrak{a} D_{n}+\delta(\mathfrak{a}) D_{n}
$$

where $\delta(f):=f(X+d X)-f(X)$ for any $f \in{ }^{b} \mathbb{C}[X]$. Evidently, there is a natural injective homomorphism of rings $A \rightarrow D(A)$ and $D(.$.$) is a covariant functor which$ conserves epimorphisms. The standard part construction gives us an epimorphism

$$
\text { st }: D(A) \longrightarrow A^{\text {an }}
$$

to the Stein algebra $A^{a n}$, associated to $A$. Let $I_{A}$ be the kernel of this map. If $A=$ ${ }^{b} \mathbb{C}[X]$, we simply write $I_{n}$. Clearly, $I_{A}$ contains each $D(A) d X_{i}$ and also $\operatorname{Inf}(A) D(A)$ where $\operatorname{Inf}(A)$ denotes, by definition, the kernel of the standard part map $A \rightarrow A^{a n}$.

Definition 6.1 We call the ideal $\mathfrak{a}$ saturated if the standard part map $\mathfrak{a} \rightarrow \overline{{ }^{\circ} \mathfrak{a}}$ is surjective.

Remark i) $\mathfrak{a}$ is saturated if ${ }^{\circ} \mathfrak{a}$ is a closed ideal in $\mathcal{O}\left(\mathbb{C}^{n}\right)$, for example if $\mathfrak{a}$ is finitely generated.

ii) If $\mathfrak{a}$ is a closed subset of ${ }^{b} \mathbb{C}[X]$ with respect to the S-topology (inherited and constructed from the compact-open topology of $\left.\mathcal{C}\left(\mathbb{C}^{n}, \mathbb{C}\right)\right)$, then $\mathfrak{a}$ is saturated.

iii) If $\mathfrak{a}$ is saturated, then the natural mappings ${ }^{i} \mathbb{C}[X] \rightarrow \operatorname{Inf}(A)$ and $I_{n} \rightarrow I_{A}$ are surjective. 
We can show now

Proposition 6.2 i) $I_{A}=\operatorname{Inf}(A)+\sum_{i=1}^{n} D(A) d X_{i}$ if $\mathfrak{a}$ is saturated,

ii) for $f \in{ }^{b} \mathbb{C}[X]$ we have, $f(X+d X)-f(X) \in I_{n}$,

iii) for $f \in{ }^{i} \mathbb{C}[X]$ we have, $f(X+d X)-f(X) \in I_{n}^{2}$.

Proof The assertion (i) is clear for $A={ }^{b} \mathbb{C}[X]$, since $P \in I_{n}$ if and only if $P_{0} \in$ ${ }^{i} \mathbb{C}[X]$. By saturation of $\mathfrak{a}$, we can reduce to the case $A={ }^{b} \mathbb{C}[X]$. For the second one, we already know that $f$ is S-continuous at each $x \in{ }^{b} \mathbb{C}^{n}$. Hence, for every $x \in{ }^{b} \mathbb{C}^{n}$ and $\xi \in{ }^{i} \mathbb{C}^{n}$, we have $f(x+\xi)-f(x) \approx 0$. The third assertion is a consequence of the stability of ${ }^{i} \mathbb{C}[X]$ under partial derivations.

Lemma 6.3 If $\mathfrak{a}$ is saturated, then we have $\operatorname{Inf}(A)^{2}=\operatorname{Inf}(A)$.

Proof By saturation, it is sufficient to treat the case $A={ }^{b} \mathbb{C}[X]$. Let $P=\sum a_{\nu} X^{\nu} \in$ ${ }^{i} \mathbb{C}[X]$. Then $a_{0} \in{ }^{i} \mathbb{C}$ and $\left|a_{\nu}\right|^{\frac{1}{|\nu|}} \in{ }^{i} \mathbb{C}$, for each $0<|\nu| \leq d$ which implies in particular that $a_{\nu} \in{ }^{i} \mathbb{C}$. Setting $\varepsilon=\max _{0 \leq|\nu| \leq d}\left|a_{\nu}\right|^{\frac{1}{2}}$, we have $\varepsilon \in{ }^{i} \mathbb{C}$, Define now

$$
Q=\sum_{0 \leq|\nu| \leq d} b_{\nu} X^{\nu} \quad \text { where } b_{\nu}:=\frac{a \nu}{\varepsilon} .
$$

It is easy to prove that $b_{0} \in{ }^{i} \mathbb{C}$ and $\left|b_{\nu}\right|^{\frac{1}{|\nu|}} \in{ }^{i} \mathbb{C}$, hence $Q \in{ }^{i} \mathbb{C}[X]$ and $P=\varepsilon Q \in$ $\left({ }^{i} \mathbb{C}[X]\right)^{2}$.

Next, we consider the canonical map $\delta_{A}: A \rightarrow D(A)$ which associates to each class $[F]$ modulo $\mathfrak{a}$ of a bounded polynomial $F \in{ }^{b} \mathbb{C}[X]$, the class $[\delta(F)]$ in $D(A)$.

Proposition 6.4 The induced mapping $\delta_{A}: A \rightarrow I_{A} / I_{A}^{2}$ is a derivation.

Proof First, we note that we can reduce immediately to the case $A={ }^{b} \mathbb{C}[X]$. But here, it is the usual standard verification.

We remark that $I_{A} / I_{A}^{2}$ carries a natural $A^{a n}$-module structure (since annihilated by $\left.\operatorname{Inf}(A) \subset I_{A}\right)$. So, we make the following definition

Definition 6.5 The *-bounded module of 1-forms is denoted by

$$
{ }^{b} \Omega_{A}:=I_{A} / I_{A}^{2} .
$$

If $A={ }^{b} \mathbb{C}[X]$, we simply write ${ }^{b} \Omega_{n}$. 
By a standard verification, we get

Proposition 6.6 If $\mathfrak{a}$ is saturated, the natural sequence of $A$-modules

$$
\mathfrak{a} / \mathfrak{a}^{2} \stackrel{\delta}{\longrightarrow}{ }^{b} \Omega_{n} / \mathfrak{a} \cdot{ }^{b} \Omega_{n} \longrightarrow{ }^{b} \Omega_{A} \rightarrow 0
$$

is exact and the three terms are $A^{a n}$-modules.

We are able to show the comparison theorem

Theorem 6.7 The natural homomorphism $\gamma_{A}:{ }^{b} \Omega_{A} \rightarrow \Omega_{A^{a n}}$ is always surjective and bijective if the ideal $\mathfrak{a}$ is saturated.

Proof We first treat the case $A={ }^{b} \mathbb{C}[X]$ and put $I:=I_{n}$. Consider the map $\varphi: I \rightarrow$ $\Gamma\left(\mathbb{C}^{n}, \Omega_{\mathbb{C}^{n}}\right)=\oplus_{i} \mathcal{O}\left(\mathbb{C}^{n}\right) d x_{i}$ defined by

$$
\varphi\left(P_{0}+\sum_{i=1}^{n} Q_{i} d X_{i}\right)=\sum_{i=1}^{n} \operatorname{st}\left(Q_{i}\right) d x_{i} .
$$

This mapping is well-defined since $Q_{i} \in D_{n}$ and we know that $\varphi\left(I^{2}\right)=0$. We shall prove that $\operatorname{Ker}(\varphi)=I^{2}$ and that $\varphi$ is surjective.

Let $P=P_{0}+\sum_{i=1}^{n} Q_{i} d X_{i} \in I$ such that $\varphi(P)=0$, so $Q_{i} \in I$. Hence, $Q_{i}=$ $Q_{i, 0}+\sum_{j=1}^{n} Q_{i j} d X_{j}$ where $Q_{i, 0} \in{ }^{i} \mathbb{C}[X]$. As a consequence, the polynomial $P$ can be expressed in the form $P=P_{0}+\sum_{i=1}^{n} Q_{i, 0} d X_{i}+\sum_{i, j=1}^{n} Q_{i j} d X_{i} d X_{j}$. By Lemma 6.3, we deduce that $P \in I^{2}$.

Now let $f(x) d x_{i}$ be an element of $\Gamma\left(\mathbb{C}^{n}, \Omega_{\mathbb{C}^{n}}\right)$ and $f_{N} \in{ }^{b} \mathbb{C}[X]$ such that ${ }^{\circ} f_{N}=f$. Consider $F_{N} \in{ }^{b} \mathbb{C}[X]$ such that $\partial^{i} F_{N}=f_{N}$. If we set

$$
P(X, d X):=F_{N}\left(X+d X_{i}\right)-F_{N}(X)
$$

we obtain $P \in I$ and

$$
P(X, d X)-f_{N}(X) d X_{i}=F_{N}\left(X+d X_{i}\right)-F_{N}(X)-f_{N}(X) d X_{i} \in I^{2} .
$$

In particular we get $\varphi(P)=\varphi\left(f_{N}(X) d X_{i}\right)=\operatorname{st}\left(f_{N}\right) d x_{i}=f(x) d x_{i}$.

The general case will be a consequence of the functorial properties of both $\Omega$-constructions:

The surjectivity of $\gamma_{A}$ is immediate. If $\mathfrak{a}$ is separated, then we can use the sequence in 6.6 and bijectivity for $A={ }^{b} \mathbb{C}[X]$ to conclude that of $\gamma_{A}$. 
Theorem 6.8 Let $\mathfrak{a}$ be saturated. Then the exact sequence of $A^{a n}$-modules

$$
0 \rightarrow I_{A} / I_{A}^{m+1} \rightarrow D(A) / I_{A}^{m+1} \rightarrow A^{a n} \rightarrow 0
$$

splits by a natural section $s: A^{a n} \rightarrow D(A) / I_{A}^{m+1}$ for any $m \in \mathbb{N}$.

Proof Consider the exact sequence of $A^{a n}$-modules

$$
0 \rightarrow I_{A} / I_{A}^{m+1} \rightarrow D(A) / I_{A}^{m+1} \rightarrow A^{a n} \rightarrow 0 .
$$

Let $s: A^{a n} \rightarrow D(A) / I_{A}^{m+1}$ be the morphism, defined by $s(f)=F \bmod I_{A}^{m+1}$, where $F \in A$ such that $\operatorname{st}(F)=f$. This map is well defined: Let $F$ and $G$ be two elements in $A$ satisfying $\operatorname{st}(F)=\operatorname{st}(G)=f$. Then $F-G \in \operatorname{Inf}(A)=\operatorname{Inf}(A)^{m+1} \subset I_{A}^{m+1}$. Clearly, $s$ is a natural section of the quotient map $D(A) / I_{A}^{m+1} \rightarrow A^{a n}$ and this implies that the above sequence of $A^{a n}$-modules is in fact split exact.

Remark It is possible to globalize our $\Omega$-construction for *-bounded schemes. But this approach is only satisfactory if the affine pieces are defined by saturated ideals.

\section{Appendix}

\section{A Filters and Ideals}

A filter is a special subset of a partially ordered set. We start by introducing a filter of sets which is the most used special case, the partially ordered set is the power set of some set. Filters play an important role in many fields of mathematics like topology in where they originated and also lattice theory. In this section, $I$ denotes a nonempty set and $\mathcal{P}(I)$ the set of subsets of $I$.

Definition A filter on I is a non-empty collection $\mathcal{F} \subset \mathcal{P}(I)$ of subsets of $I$, satisfying the following conditions

i) if $A, B \in \mathcal{F}$, then $A \cap B \in \mathcal{F}$,

ii) if $A \in \mathcal{F}$ and $A \subset B \subset I$, then $B \in \mathcal{F}$.

We say that $\mathcal{F}$ is proper if $\emptyset \notin \mathcal{F}$ that is $\mathcal{F} \subsetneq \mathcal{P}(I)$.

A trivial example of a filter on $I$ is the collection $\mathcal{F}=\{I\}$ that consists only of the set $I$ itself. 
Let $A$ be a non-empty subset of $I$. Then the collection $\mathcal{F}=\{X \subset I \mid X \supset A\}$ is a filter on $I$. It is called the principal filter on I generated by $A$. In general, if $\mathcal{H} \subset \mathcal{P}(I)$ has the finite intersection property, that is, the intersection of every nonempty finite sub-collection of $\mathcal{H}$ is non-empty, then there exist the smallest proper filter containing $\mathcal{H}$, the filter generated by $\mathcal{H}$.

An ultrafilter is a proper filter which satisfies : for any $A \subset I$, one has either $A \in \mathcal{F}$ or $I \backslash A \in \mathcal{F}$. It is an easy exercise to prove that $\mathcal{F}$ is an ultrafilter on $I$ if and only if it is a maximal proper filter on $I$, that is, there is no proper filter $\mathcal{F}^{\prime}$ on $I$ containing $\mathcal{F}$. If $A=\{a\}$, the principal filter generated by $a$ is an ultrafilter. If $I$ is finite, then every ultrafilter on $I$ is of this kind. If $I$ is infinite, there exist non-principal filters on $I$, for example the filter of all cofinite subsets of $I$.

The dual notion of a filter is an (ordered) ideal. Let us recall the definition of an ideal in a power set of some non empty set.

Definition An ideal on I is a non-empty collection $\mathfrak{J} \subset \mathcal{P}(I)$ of subsets of $I$, satisfying the following conditions

i) if $X, Y \in \mathfrak{J}$, then $X \cup Y \in \mathfrak{J}$,

ii) if $Y \in \mathfrak{J}$ and $X \subset Y$, then $X \in \mathfrak{J}$.

This definition is that of an ideal in the Boolean ring $\mathcal{P}(I)$, expressed in the terms of the operations union and intersection in the Boolean algebra $\mathcal{P}(I)$.

An ideal $\mathfrak{J}$ on $I$ is called a prime ideal if for every $X \subset I$, either $X \in \mathfrak{J}$ or $I \backslash X \in \mathfrak{J}$. In Boolean algebras, the terms prime ideal and maximal ideal coincide, as do the terms prime filter and maximal filter.

We note that if $\mathcal{F}$ is a filter on $I$ then $\mathfrak{J}=\{I \backslash X \mid X \in \mathcal{F}\}$ is an ideal, and vice versa, if $\mathfrak{J}$ is an ideal on $I$, then $\mathcal{F}=\{I \backslash X \mid X \in \mathfrak{J}\}$ is a filter.

By application of Zorn's Lemma, one proves that every proper filter on a set $I$ can be extended to an ultrafilter on $I$. So if $I$ is infinite, then there exists a non-principal ultrafilter on $I$ which contains in particular the filter of cofinite sets.

\section{Algebraic description of filters}

The following algebraic description of filters on a set $I$ is due to Kochen [12]. Let $I$ be an index set, and $K_{i}$ (where $i \in I$ ) be a family of commutative fields. We put $R:=\prod_{i \in I} K_{i}$. Then $R$ is a von Neumann regular ring, that is, it satisfies: $\forall x \in R \exists y \in R$ 
such that $x=x y x$. Kochen [12] proved that the ideal structure of the ring $R$ can be described by filters on the set $I$. We use the following notations: for $f \in R$, let $Z(f)=\{i \in I \mid f(i)=0\}$. If $\mathfrak{a}$ is any ideal in $R$, we put $\mathcal{F}(\mathfrak{a}):=\{Z(f) \mid f \in \mathfrak{a}\}$. If $\mathcal{F}$ is a filter on $I$, we define $\operatorname{Id}(\mathcal{F}):=\{f \in R \mid Z(f) \in \mathcal{F}\}$. One verifies easily that $\operatorname{Id}(\mathcal{F})$ is an ideal of $R$ and $\mathcal{F}(\mathfrak{a})$ is a filter on $I$.

Theorem The construction above gives a one-one correspondence between the family of ideals in $R$ and the family of filters on $I$. Furthermore, prime ideals correspond to ultrafilters. If $\mathfrak{a}$ is an ideal, then $R / \mathfrak{a} \simeq R / \mathcal{F}(\mathfrak{a})$. If $\mathfrak{a}$ is a prime ideal, then $R_{\mathfrak{a}} \simeq R / \mathfrak{a}$.

We want to generalize this to arbitrary commutative rings, instead of fields. We first consider the case of local rings $\left(R_{i}, \mathfrak{m}_{i}\right)_{i \in I}$, each one different from the zero ring. Put $R:=\prod_{i \in I} R_{i}$. For $f=\left(f_{i}\right)_{i} \in R$, we define the variety of $f$ by

$$
\mathrm{V}(f):=\left\{i \in I \mid f_{i} \in \mathfrak{m}_{i}\right\}
$$

If $\mathfrak{a} \subset R$ is an ideal, then $\mathcal{F}(\mathfrak{a}):=\{\mathrm{V}(f) \mid f \in \mathfrak{a}\}$ is a filter on $I$. This follows from the following facts: take $f, g \in R$, then $\mathrm{V}(f g)=\mathrm{V}(f) \cup \mathrm{V}(g)$; there are $\varepsilon, \delta \in R$, such that $\mathrm{V}(\varepsilon f+\delta g)=\mathrm{V}(f) \cap \mathrm{V}(g)$. Moreover, if $\mathfrak{a} \subset R$ is a proper ideal, then $\mathcal{F}(\mathfrak{a})$ is proper, too.

There is the dual construction: Let $\mathcal{F}$ be a filter on $I$. We put

$$
\operatorname{Id}(\mathcal{F}):=\{f \in R \mid \mathrm{V}(f) \in \mathcal{F}\} .
$$

$\operatorname{Then} \operatorname{Id}(\mathcal{F})$ is an ideal of $R$. If $\mathcal{F}$ is proper, then $\operatorname{Id}(\mathcal{F})$ is proper too. We note that both constructions conserve inclusions and we always have

$$
\begin{aligned}
\mathfrak{a} & \subset \operatorname{Id}(\mathcal{F}(\mathfrak{a})), \\
\mathcal{F} & =\mathcal{F}(\operatorname{Id}(\mathcal{F})) .
\end{aligned}
$$

One can show

Proposition i) Let $\mathfrak{m} \subset R$ be an ideal. If $\mathfrak{m}$ is maximal, then $\mathcal{F}(\mathfrak{m})$ is an ultrafilter on $I$.

ii) Let $\mathcal{F}$ be a proper filter on $I . T$ Then $\operatorname{Id}(\mathcal{F})$ is a maximal ideal of $R$ if and only if $\mathcal{F}$ is an ultrafilter on $I$.

Corollary The mapping

$$
\mathcal{F}: \operatorname{Specmax}(R) \longrightarrow \operatorname{ufil}(I)
$$

which associates to every maximal ideal of $R$ its ultrafilter, is well-defined and bijective. 
We come to the case of not necessarily local (but non zero) rings $R_{i}, i \in I$. For a commutative ring $A$, we define

$$
\mathrm{W}(A):=\prod_{\mathfrak{m} \in \operatorname{Specmax}(A)} A_{\mathfrak{m}}
$$

Then, we prove

Theorem Every maximal ideal of $R:=\Pi_{i \in I} R_{i}$ is induced by a (not necessarily unique) maximal ideal of $\Pi_{i \in I} \mathrm{~W}\left(R_{i}\right)$; that is, by an ultrafilter on the disjoint union $\sqcup_{i \in I} \operatorname{Specmax}\left(R_{i}\right)$.

For the proof, we take a maximal ideal $\mathfrak{m}$ of $R$. Put $S:=\Pi_{i \in I} \mathrm{~W}\left(R_{i}\right)$. Then we note that $\mathfrak{m} S$ is a proper ideal of $S$. Otherwise $1_{S}$ would be a finite linear combination of elements of $\mathfrak{m}$. But this implies, by the local-global principle of commutative algebra, that these elements generate each $R_{i}$, and so finally $\mathfrak{m}=R$ which is impossible. It follows that $\mathfrak{m} S$ is contained in at least one maximal ideal of $S$. We obtain our result by applying the above proposition.

\section{$\delta$-stable ultrafilters}

There are some applications in analysis where particular kinds of ultrafilter are very advantageous. In [5] Choquet constructed $\delta$-stable ultrafilters, previously called absolutely 1-simple ultrafilters in [4].

Definition [5] We say that an ultrafilter $\mathcal{U}$ is $\delta$-stable if $\left(J_{n}\right)_{n \in \mathbb{N}}$ is any sequence of elements of $\mathcal{U}$, there is a set $J_{\infty} \in \mathcal{U}$, almost contained in each $J_{n}$, so $J_{\infty} \backslash J_{n}$ is finite for each $n$.

Choquet has shown (see [4], Theorem 6 ) that under the continuum hypothesis, there exist $\delta$-stable ultrafilters over $\mathbb{N}$.

Stroyan and Luxemburg (see [18], Theorem 7.1.1 p.175) proved that each infinite natural number $\lambda$ can be represented by a sequence $\lim \lambda(j)=+\infty$. It is straightforward that this is still valid for infinite positive reals

Theorem If ${ }^{*} \mathbb{R}$ is a $\delta$ - stable ultrapower of $\mathbb{R}$ and $\lambda=[\lambda(j)] \in{ }^{*} \mathbb{R}_{\infty}^{+}$is an infinite positive real, then there exists a set $J_{\infty} \in \mathcal{U}$ such that

$$
\lim _{j \rightarrow \infty, j \in J_{\infty}} \lambda(j)=+\infty .
$$




\section{The standard part of hyperreals}

Let $\mathcal{U}$ be a non-principal ultrafilter on $\mathbb{N}$ and let ${ }^{*} \mathbb{R}$ the corresponding ultrapower of $\mathbb{R}$, that is, ${ }^{*} \mathbb{R}=\mathbb{R}^{\mathbb{N}} / \mathcal{U}$ with $\left(x_{i}\right) \sim\left(y_{i}\right)$ if $\left\{i \in \mathbb{N}, x_{i}=y_{i}\right\} \in \mathcal{U}$. Then $\left({ }^{*} \mathbb{R},+, .,<\right)$ is an ordered field extension of $(\mathbb{R},+, .,<)$. Elements of $* \mathbb{R}$ are called (hyper)real numbers.

A hyperreal $r \in{ }^{*} \mathbb{R}$ is bounded or finite if $|r|<n$ for some $n \in \mathbb{N}$, and infinitesimal if $|r|<\frac{1}{n}$ for every $n \in \mathbb{N}, n \geq 1$. Let $s \in{ }^{*} \mathbb{R}$. We say that $r$ and $s$ are infinitely close if $r-s$ is infinitesimal. We write $r \approx s$ in this case. We denote by ${ }^{b} \mathbb{R}$ the subring of ${ }^{*} \mathbb{R}$ of bounded numbers and by ${ }^{i} \mathbb{R}$ the ideal of ${ }^{b} \mathbb{R}$ of all infinitesimals.

Theorem If $\rho$ is bounded, then there exists a unique real $r$ such that $\rho \approx r$.

We call $r$ is the standard part of $\rho$ and write $r={ }^{\circ} \rho$ or $r=\operatorname{st}(\rho)$. The map

$$
\text { st : }{ }^{b} \mathbb{R} \longrightarrow \mathbb{R}
$$

is called the standard part map. It is an order preserving homomorphism from the ring ${ }^{b} \mathbb{R}$ onto $\mathbb{R}$. The kernel of "st" is ${ }^{i} \mathbb{R}$ and the quotient ring ${ }^{b} \mathbb{R} /{ }^{i} \mathbb{R}$ is isomorphic to $\mathbb{R}$. We restrict "st", the standard part map, to ${ }^{*} \mathbb{Q}$, st $_{*} \cap^{b} \mathbb{R}:{ }^{*} \cap^{b} \mathbb{R} \longrightarrow \mathbb{R}$. By density of $\mathbb{Q}$ in $\mathbb{R}, \mathrm{st}_{* \mathbb{Q} \cap b \mathbb{R}}$ is surjective and the quotient ${ }^{*} \mathbb{Q} \cap \cap^{b} \mathbb{R} /{ }^{*} \mathbb{Q} \cap \mathbb{R}$ is isomorphic to $\mathbb{R}$ (the completion of $\mathbb{Q}$ ).

We mention that Brünjes and Serpé [2] treated the case of a non trivially valued field $(K,||$.$) with locally compact completion (\hat{K},||$.$) . They proved that { }^{*} K^{\mathrm{fin}} /{ }^{*} K^{\mathrm{inf}}$ is isomorphic to $\hat{K}$, where ${ }^{*} K^{\mathrm{fin}}$ denotes the set of finite elements of ${ }^{*} K$ and ${ }^{*} K^{\text {inf }}$ the set of infinitesimal elements of ${ }^{*} K$.

\section{B Principles of Nonstandard Analysis}

\section{Transfer Principle}

The fundamental property of ultraproducts is the following:

Theorem (Łǒs) Let $L$ be a first order language and $A_{i}, i \in I$ be structures for $L$. Let $\mathcal{U}$ be an ultrafilter on $I$ and ${ }^{*} A=\left(\prod_{i \in I} A_{i}\right) / \mathcal{U}$. Then for any first order formula $\varphi\left(x_{1}, \ldots, x_{n}\right)$ with $x_{1}, \ldots, x_{n}$ its only free variables and $\left[a_{1}\right], \ldots,\left[a_{n}\right] \in{ }^{*} A$, we have that ${ }^{*} \varphi\left(\left[a_{1}\right], \ldots,\left[a_{n}\right]\right)$ is true in ${ }^{*} A$ if and only if $\left\{i \in I: \varphi\left(a_{1}(i), \ldots, a_{n}(i)\right)\right.$ is true in $\left.A_{i}\right\} \in$ $\mathcal{U}$. In particular, if $\varphi$ is a sentence, then $\varphi$ is true if and only if ${ }^{*} \varphi$ is true. 
We present some interesting applications of Łǒs’ Theorem.

i) Let $\left(k_{i}\right)_{i \in I}$ be a family of (algebraically closed) fields. Then ${ }^{*} k$, their ultraproduct, is again a (algebraically closed) field.

If for each prime $p$, only finitely many $k_{i}$ have characteristic $p$, then ${ }^{*} k$ has characteristic zero. In particular, if $P$ is an infinite prime in ${ }^{*} \mathbb{Z}$, then ${ }^{*} \mathbb{Z} / P^{*} \mathbb{Z}$ is a field of characteristic zero. Its algebraic closure is (non-canonically) isomorphic to $\mathbb{C}$, because each field is algebraically closed with the same cardinality which is equal to that of the continuum. In the same manner, one can show that ${ }^{*} \mathbb{C}$ is (non-canonically) isomorphic to $\mathbb{C}$.

ii) Let $\left(A_{i}\right)_{i \in I}$ be a family of local rings with maximal ideal $\mathfrak{m}_{i}$ and residue field $k_{i}=$ $A_{i} / \mathfrak{m}_{i}$. Then ${ }^{*} A$, their ultraproduct, is a local ring with maximal ideal ${ }^{*} \mathfrak{m}$ and residue field ${ }^{*} k={ }^{*} A /{ }^{*} \mathfrak{m}$. In fact, a ring is local if and only if the sum of two non-units is a non unit.

\section{Permanence Principle}

This principle asserts that certain functions can be extended to larger domains than those over which they are originally defined.

Theorem Let $\varphi(x)$ be an internal formula with the only free variable $x$. Then

i) If there exists $k \in \mathbb{N}$ such that $\varphi(n)$ is true for all $n \in \mathbb{N}$ with $k \leq n$, then there exists $K \in{ }^{*} \mathbb{N} \backslash \mathbb{N}$ such that $\varphi(n)$ is true for all $n \in{ }^{*} \mathbb{N}$ with $k \leq n \leq K$.

ii) If there exists $K \in{ }^{*} \mathbb{N} \backslash \mathbb{N}$ such that $\varphi(n)$ is true for all $n \in{ }^{*} \mathbb{N} \backslash \mathbb{N}$ with $n \leq K$, then there exists $k \in \mathbb{N}$ such that $\varphi(n)$ is true for all $n \in{ }^{*} \mathbb{N}$ with $k \leq n \leq K$.

iii) If $\varphi(x)$ holds for each infinitesimal $x$, then there is a standard $r>0$ in $\mathbb{R}$ so that $\varphi(b)$ holds for all $b$ with $|b| \leq r$ in $* \mathbb{R}$.

Corollary (Spillover Principle) Let $A$ be an internal subset of ${ }^{*} \mathbb{R}$.

i) If A contains all standard natural numbers, then $A$ contains an infinite natural number.

ii) If $A$ contains all infinite natural numbers, then $A$ contains a standard natural number. 


\section{Concurrence or Saturation Principle}

This principle provides us with new objects in nonstandard extensions. Let $X$ be an infinite set and $R$ be a binary relation on $X . R$ is called concurrent if for any finite subset $\left\{x_{1}, \ldots, x_{n}\right\}$ of the domain of $R$ there exists an element $y$ with $x_{i} R y$ for all $\mathrm{i}$ between 1 and $n$.

Theorem If $R$ is concurrent, then there exists $b \in{ }^{*} X$ such that ${ }^{*} x\left({ }^{*} R\right) b$ for all $x \in$ $\operatorname{dom} R$.

This is also called the enlargement property: ${ }^{*} X$ is an enlargement of $X$ if every concurrent relation verifies the enlargement property. Let us consider some examples:

i) $R(x, y):(x \in \mathbb{R}) \wedge(y \in \mathbb{R})(x \leq y) R$ is a concurrent relation on $\mathbb{R}$, so there exists $b \in{ }^{*} \mathbb{R}$ such that $x \leq b$ for any $x \in \mathbb{R}$. Evidently, $b$ is an infinite number.

ii) Let $X$ be a topological space, $x \in X$ and ${ }^{*} X$ an enlargement of $X$. Then there exists an internal open set $W$ in ${ }^{*} X$ containing $x$, such that $W \subset \mu(x)$.

We denote by $\Omega_{x}$ the system of open neighborhoods of $x$. The binary relation $R$ defined on $\Omega_{x} \times \Omega_{x}$ defined by $R(U, V)$ if $U \supset V$ is concurrent. So, the enlargement property guarantees the existence of $W \in{ }^{*} \Omega_{x}$ such that ${ }^{*} U \supset W$, for every $U \in \Omega_{x}$. In a metric space, one can take $W$ an $*$-ball with $x$ as center and positive infinitesimal radius.

\section{Nonstandard Topologies}

\section{Topological spaces}

Let $(X, \mathcal{T})$ be a topological space. In the literature, usually two topologies on the nonstandard extension of ${ }^{*} X$ are considered. The first one, called $Q$-topology, introduced by Robinson [15], has a basis, consisting of elements in ${ }^{*} \mathcal{T}$. In fact, by transfer, elements of $* \mathcal{T}$ are stable under $*$-finite intersection, hence under finite intersection and under internal union. Furthermore, $\emptyset,{ }^{*} X \in{ }^{*} \mathcal{T}$. Elements in the $Q$-topology are called $Q$-open and elements in the base ${ }^{*} \mathcal{T}$ are called $*$-open subsets of ${ }^{*} X$. A $*$-open set is clearly $Q$-open. The converse is false in general: there are external sets which are open in this topology. Robinson proved that if $Y$ is an internal subset of ${ }^{*} X$, then $Y$ is $Q$-open if and only if $Y$ is $*$-open. The second one, called $S$-topology by Robinson, has as basis $\mathcal{B}=\left\{{ }^{*} U \mid U \in \mathcal{T}\right\}$. The $Q$-topology is finer than the $S$-topology.

Let $x \in X$ be a point. The monad of $x$ or halo is the subset $\mu(x)=\cap_{U \in \mathcal{T}, x \in U^{*} U}$. 
A point $y \in{ }^{*} X$ is, by definition, near-standard, if there exists $x \in X$ such that $y \in \mu(x)$. We write $y \approx x$ in this case. The set of near-standard points of ${ }^{*} X$ is $\mathrm{ns}\left({ }^{*} \mathrm{X}\right)=\cup_{x \in X} \mu(x)$. Many topological properties of $X$ can be expressed via monads, so the halos of ${ }^{*} X$ encode the topology of $X$.

For a Hausdorff topological space, we have a standard part map st : ns $\left({ }^{*} \mathrm{X}\right) \rightarrow X$ defined as follows: we set $\operatorname{st}(y)={ }^{\circ} y=x$, for every $y \in \mu(x)$ and $x \in X$. This map is well-defined because in the case of Hausdorff spaces, halos of standard points constitute a disjoint partition of near standard points. Even for a non-Hausdorff space, we can define the standard part of a set $B \subset{ }^{*} X$, by setting:

$$
\operatorname{st}(B)={ }^{\circ} B=\{x \in X \mid \mu(x) \cap B \neq \emptyset\} .
$$

Under certain cardinality restrictions (expressed via the notion " $\kappa$-saturated enlargement"), standard parts of internal subsets turn out to be always closed. The following result can be found in the book of Hurd and Loeb ([10] p.117)

Theorem Assume that $(X, \mathcal{T})$ is a topological space and ${ }^{*} \mathcal{T}$ is a $\kappa$-saturated enlargement of $\mathcal{T}$ with $\kappa>$ card $\mathcal{T}$.

i) If $B$ is internal subset of ${ }^{*} X$, then $\operatorname{st}(B)$ is closed.

ii) If $B$ is internal subset of near-standard points of ${ }^{*} X$, then $\operatorname{st}(B)$ is compact.

If $X$ satisfies the first axiom of countability (for example if $X$ is a metric space), one can use just $\aleph_{1}$-saturation.

\section{Metric spaces}

Let $X$ be a metric space with distance function $\rho$ and $\Gamma$ be the set of all open balls B, where $B(x, r)=\{y \in X \mid \rho(x, y)<r\}$ for a point $x \in X$ and a positive real $r$. We describe the monad of a point $x \in{ }^{*} X$ by $\mu(x)=\cap^{*} B(x, r)=\left\{y \in{ }^{*} X \mid{ }^{*} \rho(x, y) \approx 0\right\}$. Obviously, ${ }^{*} \Gamma$ forms a basis for the $Q$-topology of ${ }^{*} X$. Let $p \in{ }^{*} X$ and $r$ a standard positive number. We put $S(p, r)=\left\{q \in{ }^{*} X \mid{ }^{\circ} \rho(p, q)<r\right\}$. The $S$-balls give us a topology in ${ }^{*} X$, which is the $S$-topology. The space ${ }^{*} X$, endowed with the $S$-topology in not Hausdorff. In fact, if $x, y \in{ }^{*} X$ such that $x \approx y$ and if $r>0$ is a standard positive real, we obtain $S(x, r)=S(y, r)$.

Let $(X, \rho),(Y, d)$ be two metric spaces and $f:{ }^{*} X \rightarrow{ }^{*} Y$ be a map from ${ }^{*} X$ to ${ }^{*} Y$. Let $x \in{ }^{*} X$ be a point. We say that $f$ is $S$-continuous at $x$, if $f$ is continuous at $x$ as a map from ${ }^{*} X$ to ${ }^{*} Y$ where both sets are equipped with the $S$-topology, that is, for every 
standard $\varepsilon>0$, there exists a standard $\delta>0$ such that ${ }^{*} d(f(x), f(y))<\varepsilon$ for each $y \in{ }^{*} X$, such that ${ }^{*} \rho(x, y)<\delta$. There is a simple characterization of $S$-continuity of internal maps. If $f$ is an internal map, then $f$ is $S$-continuous at $x$ if and only if $f(\mu(x)) \subset \mu(f(x))$.

Let $X$ be a metric space with distance function $\rho$. A point $p \in{ }^{*} X$ is called bounded (or finite) if there exists a standard point $q \in X$ such that ${ }^{*} \rho(p, q)$ is bounded; that is, there exists a standard positive real $m>0$ such that $p \in{ }^{*} B(q, m)$. We denote by bd( $\left.{ }^{*} X\right)$ the set of bounded points of ${ }^{*} X$. Robinson proved (see [15] Theorem 4.3.1 p.100) that a metric space $X$ is bounded if and only if ${ }^{*} X=\operatorname{bd}\left({ }^{*} X\right)$. Clearly, we always have $\mathrm{ns}\left({ }^{*} X\right) \subset \operatorname{bd}\left({ }^{*} X\right)$.

\section{Standard part of a map}

Let $X$ and $Y$ be two topological spaces. $\mathcal{F}(X, Y)$ (resp. $\mathcal{C}(X, Y)$ ) denotes the set of all (resp. continuous) maps from $X$ to $Y$. As usual ns( $\left.{ }^{*} X\right)$ is the set of nearstandard points and $\operatorname{cpt}\left({ }^{*} X\right)=\cup_{K \subset X, K \text { compact }}{ }^{*} K$ is the set of "compact points".

One of the most powerful tools in nonstandard analysis is taking standard parts of objects of the nonstandard universe. If $Y$ is Hausdorff, we can define for every $f \in$ ${ }^{*} \mathcal{C}(X, Y)$ with $f\left({ }^{*} x\right) \in \mathrm{ns}\left({ }^{*} X\right)$ for all $x \in X$, the standard part function st $f: X \rightarrow Y$ by $\operatorname{st} f(x):={ }^{\circ}\left(f\left({ }^{*} x\right)\right)$. Thus, we have a mapping

$$
\text { st }:\left\{f \in{ }^{*} \mathcal{C}(X, Y) \mid f\left({ }^{*} x\right) \in \mathrm{ns}\left({ }^{*} Y\right) \forall x \in X\right\} \longrightarrow \mathcal{F}(X, Y)
$$

It is known that st $f$ is, in general, not a continuous function.

Theorem Let $B$ be a subset of ${ }^{*} X$ and $f: B \rightarrow{ }^{*} Y$ an internal map such that $f(B) \subset \mathrm{ns}\left({ }^{*} Y\right)$ and $f$ is $S$-continuous on $B$. Then st $f$ exists and is continuous on ${ }^{\circ} B$.

We endow $\mathcal{C}(X, Y)$ with $\tau$, the compact open topology. Let $f \in \mathcal{C}(X, Y)$ be a mapping. We denote by $\mu_{\tau}(f)$ the halo of $f$ with respect to the compact open topology. One easily verifies that

$$
\mu_{\tau}(f)=\left\{g \in{ }^{*} \mathcal{C}(X, Y) \mid g(x) \approx{ }^{*} f(x) \forall x \in \operatorname{cpt}\left({ }^{*} X\right)\right\}
$$

If $X$ is locally compact, then $\operatorname{cpt}\left({ }^{*} X\right)=\mathrm{ns}\left({ }^{*} X\right)$, hence

$$
\mu_{\tau}(f)=\left\{g \in{ }^{*} \mathcal{C}(X, Y) \mid g(x) \approx{ }^{*} f(x) \forall x \in \mathrm{ns}\left({ }^{*} X\right)\right\} .
$$


Theorem Let $X, Y$ be two Hausdorff topological spaces and $X$ locally compact. Then $\mathrm{ns}_{\tau}\left({ }^{*} \mathcal{C}(X, Y)\right)$ consists of all internal maps sending nearstandard points of ${ }^{*} X$ to nearstandard points of ${ }^{*} Y$ and which are $S$-continuous on $n s\left({ }^{*} X\right)$.

In particular, nearstandard functions for the compact-open topology are those which are bounded and $S$-continuous on $\mathrm{ns}\left({ }^{*} X\right)$.

\section{References}

[1] L. Brünjes, C. Serpé, Enlargements of Categories, Theory and Applications of Categories, 14(2005), 357-398; http://www.tac.mta.ca/tac/volumes/14/16/ 14-16abs.html.

[2] L. Brünjes, C. Serpé, Enlargements of schemes, Logic and Analysis 1(2007), 1-60; doi: 10.1007/s11813-007-0001-x.

[3] J.L. Callot, Calcul infinitésimal complexe, available at: http://math1.unice.fr/ rgr/prepubli.htm.

[4] G. Choquet, Construction d'ultrafiltres sur $\mathbb{N}$, Bull. Sci. Math. 92(2)(1968), 41-48.

[5] G. Choquet, Deux classes remarquables d'ultrafiltres sur $\mathbb{N}$, Bull. Sci. Math. 92(2)(1968), 143-153.

[6] O. Forster, Primärzerlegung in Steinschen Algebren, Math. Ann. 154(1964), 307-329.

[7] A. Fruchard, Complex analysis, In F.Diener, M.Diener, Nonstandard Analysis in Practice, Springer Verlag 1995.

[8] R. Goldblatt, Lectures on the Hyperreals: An Introduction to Nonstandard Analysis, Graduate Texts in Mathematics 188, Springer Verlag 1998.

[9] K. Hahn, K. Tim, Hyperbolicity of complex manifolds and other equivalent properties. Proc. Amer. Math. Soc. 91(1984), 49-53; doi:10.1090/S0002-9939-19840735562-9.

[10] A. Hurd, P. Loeb, An Introduction to Nonstandard Real Analysis, Academic Press, Orlando 1985.

[11] S. Kobayashi, Hyperbolic Complex Spaces, Springer Verlag 1998.

[12] S. Kochen, Ultraproducts in the theory of models, Ann.Math. 74, No. 2(1961), 221261.

[13] A. Lightstone, A. Robinson, Nonarchimedean Fields and Asymptotic Expansions, North-Holland, Amsterdam 1975.

[14] R. Lutz, M. Goze, Nonstandard Analysis: A practical guide with applications, Lect.Notes in Math. 881, Springer Verlag 1981.

[15] A. Robinson, Non-Standard Analysis, North-Holland, Amsterdam 1966. 
[16] A. Robinson, Germs, in Applications of Model Theory to Algebra, Analysis, and Probability (Internat.Sympos., Pasadena, Calif., 1967), pp. 138-149, Holt, Rinehart and Winston, New-York.

[17] Y.T. Siu, Hilbert Nullstellensatz in global complex-analytic case, Proc. Amer. Math. Soc. Vol. 19 No. 2 (1968), 296-298; doi:10.1090/S0002-9939-1968-0222339-7.

[18] K.D. Stroyan, W.A.J. Luxemburg, Introduction to the theory of infinitesimals, Academic Press, New York 1976.

[19] G. Wallet, Nonstandard generic points, Bull. Belg. Math. Soc. Simon Stevin 13(5)(2006), 1033-1057.

Department of Mathematics, Faculty of Science, King Khalid University, Abha

P.O.Box 9004, Saudi Arabia

Institut Fourier Université de Grenoble 1

100 rue des Mathématiques, 38402 Saint Martin d'Hères, France

a_khalfallah@yahoo.fr, siegmund.kosarew@ujf-grenoble.fr

Received: 9 November 2008 Revised: 5 July 2010 